\title{
A history of solar activity over millennia
}

\author{
Ilya G. Usoskin ${ }^{1}$
}

Received: 6 September 2016 / Accepted: 3 February 2017 / Published online: 4 March 2017 (C) The Author(s) 2017. This article is an open access publication

\begin{abstract}
Presented here is a review of present knowledge of the long-term behavior of solar activity on a multi-millennial timescale, as reconstructed using the indirect proxy method. The concept of solar activity is discussed along with an overview of the special indices used to quantify different aspects of variable solar activity, with special emphasis upon sunspot number. Over long timescales, quantitative information about past solar activity can only be obtained using a method based upon indirect proxies, such as the cosmogenic isotopes ${ }^{14} \mathrm{C}$ and ${ }^{10} \mathrm{Be}$ in natural stratified archives (e.g., tree rings or ice cores). We give an historical overview of the development of the proxy-based method for past solar-activity reconstruction over millennia, as well as a description of the modern state. Special attention is paid to the verification and cross-calibration of reconstructions. It is argued that this method of cosmogenic isotopes makes a solid basis for studies of solar variability in the past on a long timescale (centuries to millennia) during the Holocene. A separate section is devoted to reconstructions of strong solar energetic-particle (SEP) events in the past, that suggest that the present-day average SEP flux is broadly consistent with estimates on longer timescales, and that the occurrence of extra-strong events is unlikely. Finally, the main features of the long-term evolution of solar magnetic activity, including the
\end{abstract}

This article is a revised version of http://dx.doi.org/10.12942/lrsp-2013-1.

Change summary Major revision, updated and expanded.

Change details Several major updates have been made, in particular, about recent updates of the sunspot number series, new multi-millennial solar activity reconstructions, including long-period cycles, Grand minima and maxima of solar activity, the extreme SEP even of $775 \mathrm{AD}$, about nitrate in ice cores as a possible index of solar variability. The number of references has increased from 401 to 474 .

$凶 \quad$ Ilya G. Usoskin

ilya.usoskin@oulu.fi

1 Sodankylä Geophysical Observatory (Oulu Unit), University of Oulu, 90014 Oulu, Finland 
statistics of grand minima and maxima occurrence, are summarized and their possible implications, especially for solar/stellar dynamo theory, are discussed.

Keywords Solar activity · Paleo-astrophysics · Cosmogenic isotopes · Solarterrestrial relations $\cdot$ Solar physics $\cdot$ Long-term reconstructions $\cdot$ Solar dynamo

\section{Contents}

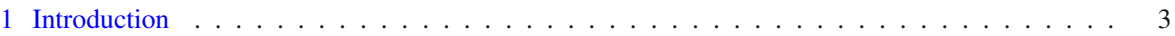

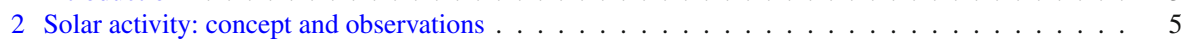

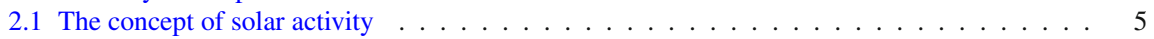

2.2 Indices of solar activity . . . . . . . . . . . . . . . . . . . . 5

2.2 .1 Direct solar indices . . . . . . . . . . . . . . . . . . . . 6

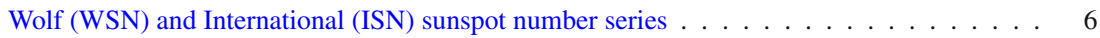

Group sunspot number $(\mathrm{GSN})$ series $\ldots \ldots \ldots \ldots \ldots$

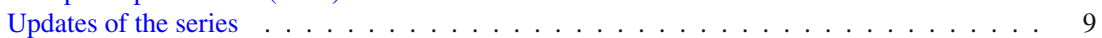

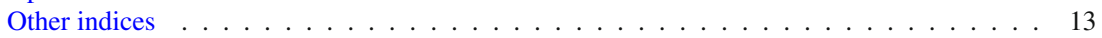

2.2 .2 Indirect indices . . . . . . . . . . . . . . . . . . . . . . . . . . . . . . . . . . .

2.3 Solar activity observations in the pre-telescopic epoch . . . . . . . . . . . . . . . . 15

2.3.1 Instrumental observations: camera obscura . . . . . . . . . . . . . . . . . . 16

2.3.2 Naked-eye observations . . . . . . . . . . . . . . . . . . . 16

2.3.3 Mathematical/statistical extrapolations . . . . . . . . . . . . . . . . . . . 17

2.4 The solar cycle and its variations . . . . . . . . . . . . . . . . . . . . . . . . . . . . . . . . . . . . . . . . . . .

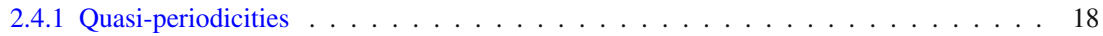

11 -years Schwabe cycle . . . . . . . . . . . . . . . . . . . . . . . . . . . . . . . . . . . . . .

Phase catastrophe $\quad \ldots \ldots \ldots \ldots \ldots$. . . . . . . . . . . . . . . . . . . . . . . 19

Centennial Gleissberg cycle . . . . . . . . . . . . . . . . . . . . . . . . . 19

210-years Suess/de Vries cycle . . . . . . . . . . . . . . . . . . . . . . . . . 19

Millennial Eddy cycle . . . . . . . . . . . . . . . . . . . . . . . . . 20

$\approx 2400$-years Hallstatt cycle . . . . . . . . . . . . . . . . . . . . . . . . 20

2.4 .2 Randomness versus regularity . . . . . . . . . . . . . . . . . . . . . . . 20

2.4 .3 A note on solar activity predictions . . . . . . . . . . . . . . . . . 22

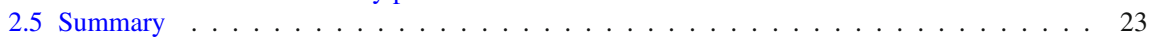

3 The proxy method of past solar-activity reconstruction . . . . . . . . . . . . . . . . . 24

3.1 The physical basis of the method . . . . . . . . . . . . . . . . . . . 25

3.1.1 Heliospheric modulation of cosmic rays . . . . . . . . . . . . . . . . . 25

3.1 .2 Geomagnetic shielding . . . . . . . . . . . . . . . . . . . . . 27

3.1.3 Cosmic-ray-induced atmospheric cascade . . . . . . . . . . . . . . . . . . . . . 29

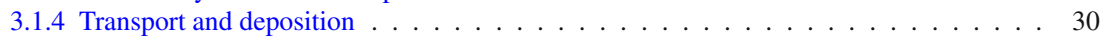

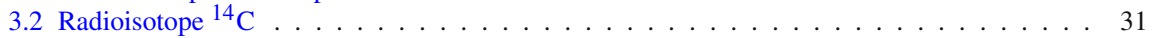

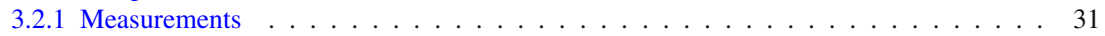

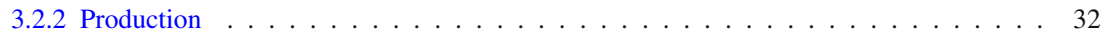

3.2 .3 Transport and deposition . . . . . . . . . . . . . . . . . . . . 35

3.2 .4 The Suess effect and nuclear bomb tests . . . . . . . . . . . . . . . . . 38

3.2 .5 The effect of the geomagnetic field . . . . . . . . . . . . . . . . . 38

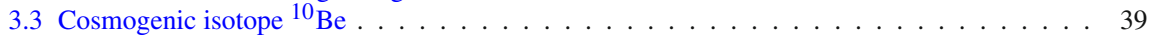

3.3 .1 Measurements . . . . . . . . . . . . . . . . . . . . 39

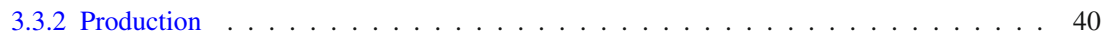

3.3 .3 Atmospheric transport . . . . . . . . . . . . . . . . . . . . 41

3.3 .4 Effect of the geomagnetic field . . . . . . . . . . . . . . . . . . . . . . . . . . . . . . . .

3.4 Other potential proxy . . . . . . . . . . . . . . . . . . . . . . . . 43

3.5 Towards a quantitative physical model . . . . . . . . . . . . . . . . . . . . . . . . . . . . . . . . . .

3.5.1 Regression models . . . . . . . . . . . . . . . . . . . . . . 45

3.5.2 Reconstruction of heliospheric parameters . . . . . . . . . . . . . . . . . . 46

3.5 .3 A link to sunspot numbers . . . . . . . . . . . . . . . . . . . . 48 
3.6 Solar activity reconstructions . . . . . . . . . . . . . . . . . . . . . . . . . 49

3.7 Verification of reconstructions . . . . . . . . . . . . . . . . . . . 50

3.7 .1 Comparison with direct data . . . . . . . . . . . . . . . . . . 50

3.7.2 Meteorites and lunar rocks: A direct probe of the galactic cosmic-ray flux . . . . . . . 51

3.7 .3 Comparison between isotopes . . . . . . . . . . . . . . . . 52

3.8 Composite reconstruction . . . . . . . . . . . . . . . . . . . . 53

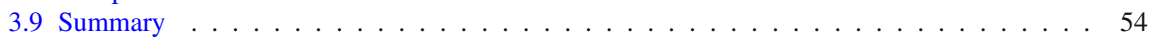

4 Variability of solar activity over millennia . . . . . . . . . . . . . . . . . . . . 55

4.1 Quasi-periodicities and characteristic times . . . . . . . . . . . . . . . . 55

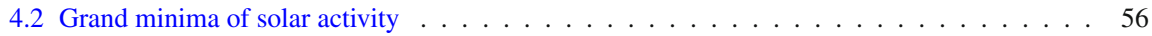

4.2 .1 The Maunder minimum _. . . . . . . . . . . . . . . . . . 56

4.2 .2 Grand minima on a multi-millennial timescale . . . . . . . . . . . . . . . . . . . 58

4.3 Grand maxima of solar activity . . . . . . . . . . . . . . . . . . . . . . . . . 62

4.3.1 The modern episode of active sun . . . . . . . . . . . . . . . . . . 62

4.3.2 Grand maxima on a multi-millennial timescale . . . . . . . . . . . . . 62

4.4 Related implications . . . . . . . . . . . . . . . . . . . . . . . . . 64 . . . . . . . . . . . . . 64

4.4 Theoretical constrains . . . . . . . . . . . . . . . . . . . 64

4.4 .2 Solar-terrestrial relations . . . . . . . . . . . . . . . . . . . . 65

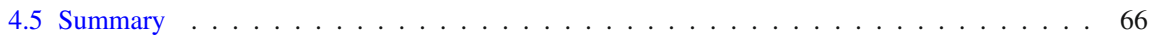

5 Solar energetic particles in the past . . . . . . . . . . . . . . . . . . . . 67

5.1 Cosmogenic isotopes . . . . . . . . . . . . . . . . . . . 68

5.1.1 The event of 775 AD: the worst case scenario? . . . . . . . . . . . . . 69

5.1 .2 Occurrence rate . . . . . . . . . . . . . . . . . . . 71

5.2 Lunar and meteoritic rocks . . . . . . . . . . . . . . . . . . . . . . . . . 72

5.3 Nitrates in polar ice . . . . . . . . . . . . . . . . . . . . . . . . 74

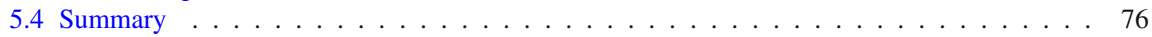

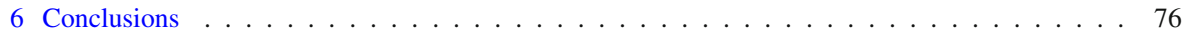

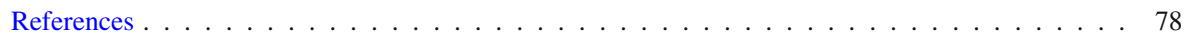

\section{Introduction}

The concept of the perfectness and constancy of the sun, postulated by Aristotle, was a strong belief for centuries and an official doctrine of Christian and Muslim countries. However, as people had noticed already before the time of Aristotle, some slight transient changes of the sun can be observed even with the naked eye. Although scientists knew about the existence of "imperfect" spots on the sun since the early seventeenth century, it was only in the nineteenth century that the scientific community recognized that solar activity varies in the course of an 11-year solar cycle. Solar variability was later found to have many different manifestations, including the fact that the "solar constant", or the total solar irradiance, TSI, (the amount of total incoming solar electromagnetic radiation in all wavelengths per unit area at the top of the atmosphere) is not a constant. The sun appears much more complicated and active than a static hot plasma ball, with a great variety of nonstationary active processes going beyond the adiabatic equilibrium foreseen in the basic theory of sun-as-star. Such transient nonstationary (often eruptive) processes can be broadly regarded as solar activity, in contrast to the so-called "quiet" sun. Solar activity includes active transient and long-lived phenomena on the solar surface, such as spectacular solar flares, sunspots, prominences, coronal mass ejections (CMEs), etc.

The very fact of the existence of solar activity poses an enigma for solar physics, leading to the development of sophisticated models of an upper layer known as the convection zone and the solar corona. The sun is the only star, which can be studied 
in great detail and thus can be considered as a proxy for cool stars. Quite a number of dedicated ground-based and space-borne experiments are being carried out to learn more about solar variability. The use of the sun as a paradigm for cool stars leads to a better understanding of the processes driving the broader population of cool sun-like stars. Therefore, studying and modelling solar activity can increase the level of our understanding of nature.

On the other hand, the study of variable solar activity is not of purely academic interest, as it directly affects the terrestrial environment. Although changes in the sun are barely visible without the aid of precise scientific instruments, these changes have great impact on many aspects of our lives. In particular, the heliosphere (a spatial region of about 200-300 astronomical units across) is mainly controlled by the solar magnetic field. This leads to the modulation of galactic cosmic rays (GCRs) by the solar magnetic activity. Additionally, eruptive and transient phenomena in the sun/corona and in the interplanetary medium can lead to sporadic acceleration of energetic particles with greatly enhanced flux. Such processes can modify the radiation environment on Earth and need to be taken into account for planning and maintaining space missions and even transpolar jet flights. Solar activity can cause, through coupling of solar wind and the Earth's magnetosphere, strong geomagnetic storms in the magnetosphere and ionosphere, which may disturb radio-wave propagation and navigation-system stability, or induce dangerous spurious currents in long pipes or power lines. Another important aspect is the link between solar-activity variations and the Earth's climate (see, e.g., reviews by Haigh 2007; Gray et al. 2010; Mironova et al. 2015).

It is important to study solar variability on different timescales. The primary basis for such studies is observational (or reconstructed) data. The sun's activity is systematically explored in different ways (solar, heliospheric, interplanetary, magnetospheric, terrestrial), including ground-based and space-borne experiments and dedicated missions during the last few decades, thus covering 3-4 solar cycles. However, it should be noted that the modern epoch was characterized, until the earlier 2000s by high solar activity dominated by an 11-year cyclicity, and it is not straightforward to extrapolate present knowledge (especially empirical and semi-empirical relationships and models) to a longer timescale. The current cycle 24 indicates the return to the normal moderate level of solar activity, as manifested, e.g., via the extended and weak solar minimum in 2008-2009 and weak solar and heliospheric parameters, which are unusual for the space era but may be quite typical for the normal activity (see, e.g., Gibson et al. 2011). On the other hand, contrary to some predictions, a Grand minimum of activity has no started. Thus, we may experience, in the near future, the interplanetary conditions quite different with respect to those we got used to during the last decades.

Therefore, the behavior of solar activity in the past, before the era of direct measurements, is of great importance for a variety of reasons. For example, it allows an improved knowledge of the statistical behavior of the solar-dynamo process, which generates the cyclically-varying solar-magnetic field, making it possible to estimate the fractions of time the sun spends in states of very-low activity, what are called grand minima. Such studies require a long time series of solar-activity data. The longest direct series of solar activity is the 400-year-long sunspot-number series, which depicts the dramatic contrast between the (almost spotless) Maunder minimum and the modern period of very high activity. Thanks to the recent development of precise technologies, 
including accelerator mass spectrometry, solar activity can be reconstructed over multiple millennia from concentrations of cosmogenic isotopes ${ }^{14} \mathrm{C}$ and ${ }^{10} \mathrm{Be}$ in terrestrial archives. This allows one to study the temporal evolution of solar magnetic activity, and thus of the solar dynamo, on much longer timescales than are available from direct measurements.

This paper gives an overview of the present status of our knowledge of long-term solar activity, covering the period of Holocene (the last 11 millennia). A description of the concept of solar activity and a discussion of observational methods and indices are presented in Sect. 2. The proxy method of solar-activity reconstruction is described in some detail in Sect. 3. Section 4 gives an overview of what is known about past solar activity. The long-term averaged flux of solar energetic particles is discussed in Sect. 5. Finally, conclusions are summarized in Sect. 6.

\section{Solar activity: concept and observations}

\subsection{The concept of solar activity}

The sun is known to be far from a static state, the so-called "quiet" sun described by simple stellar-evolution theories, but instead goes through various nonstationary active processes. Such nonstationary and nonequilibrium (often eruptive) processes can be broadly regarded as solar activity. The presence of magnetic activity, including stellar flares, is considered as a common typical feature of sun-like stars (Maehara et al. 2012). Although a direct projection of the energy and occurrence frequency of superflares on sun-like stars (e.g.,Shibata et al. 2013) does not agree with solar data (Aulanier et al. 2013) and terrestrial proxy (see Sect. 5), the existence of solar/stellar activity is clear. Whereas the concept of solar activity is quite a common term nowadays, it is neither straightforwardly interpreted nor unambiguously defined. For instance, solar-surface magnetic variability, eruption phenomena, coronal activity, radiation of the sun as a star or even interplanetary transients and geomagnetic disturbances can be related to the concept of solar activity. A variety of indices quantifying solar activity have been proposed in order to represent different observables and caused effects. Most of the indices are highly correlated to each other due to the dominant 11-year cycle, but may differ in fine details and/or long-term trends. In addition to the solar indices, indirect proxy data is often used to quantify solar activity via its presumably known effect on the magnetosphere or heliosphere. The indices of solar activity that are often used for long-term studies are reviewed below.

\subsection{Indices of solar activity}

Solar (as well as other) indices can be divided into physical and synthetic according to the way they are obtained/calculated. Physical indices quantify the directly-measurable values of a real physical observable, such as, e.g., the radioflux, and thus have clear physical meaning as they quantify physical features of different aspects of solar activity and their effects. Synthetic indices (the most common being sunspot number) are calculated (or synthesized) using a special algorithm from observed (often not mea- 
surable in physical units) data or phenomena. Additionally, solar activity indices can be either direct (i.e., directly relating to the sun) or indirect (relating to indirect effects caused by solar activity), as discussed in subsequent Sects. 2.2.1 and 2.2.2.

\subsubsection{Direct solar indices}

The most commonly used index of solar activity is based on sunspot number. Sunspots are dark areas on the solar disc (of size up to tens of thousands of $\mathrm{km}$, lifetime up to halfa-year), characterized by a strong magnetic field, which leads to a lower temperature (about $4000 \mathrm{~K}$ compared to $5800 \mathrm{~K}$ in the photosphere) and observed as darkening.

Sunspot number is a synthetic, rather than a physical, index, but it has still become quite a useful parameter in quantifying the level of solar activity. This index presents the weighted number of individual sunspots and/or sunspot groups, calculated in a prescribed manner from simple visual solar observations. The use of the sunspot number makes it possible to combine together thousands and thousands of regular and fragmentary solar observations made by earlier professional and amateur astronomers. The technique, initially developed by Rudolf Wolf, yielded the longest series of directly and regularly-observed scientific quantities. Therefore, it is common to quantify solar magnetic activity via sunspot numbers. For details see the review on sunspot numbers and solar cycles (Hathaway and Wilson 2004; Hathaway 2015).

\section{Wolf (WSN) and International (ISN) sunspot number series}

The concept of the sunspot number was developed by Rudolf Wolf of the Zürich observatory in the middle of the nineteenth century. The sunspot series, initiated by him, is called the Zürich or Wolf sunspot number (WSN) series. The relative sunspot number $R_{z}$ is defined as

$$
R_{z}=k(10 G+N),
$$

where $G$ is the number of sunspot groups, $N$ is the number of individual sunspots in all groups visible on the solar disc and $k$ denotes the individual correction factor, which compensates for differences in observational techniques and instruments used by different observers, and is used to normalize different observations to each other.

The value of $R_{z}$ (see Fig. 1a) is calculated for each day using only one observation made by the "primary" observer (judged as the most reliable observer during a given time) for the day. The primary observers were Staudacher (1749-1787), Flaugergues (1788-1825), Schwabe (1826-1847), Wolf (1848-1893), Wolfer (1893-1928), Brunner (1929-1944), Waldmeier (1945-1980) and Koeckelenbergh (since 1980). If observations by the primary observer are not available for a certain day, the secondary, tertiary, etc. observers are used (see the hierarchy of observers in Waldmeier 1961). The use of only one observer for each day aims to make $R_{z}$ a homogeneous time series. As a drawback, such an approach ignores all other observations available for the day, which constitute a large fraction of the existing information. Moreover, possible errors of the primary observer cannot be caught or estimated. The observational uncertainties in the monthly $R_{z}$ can be up to $25 \%$ (e.g., Vitinsky et al. 1986). The WSN series is based on observations performed at the Zürich Observatory during 1849-1981 using 


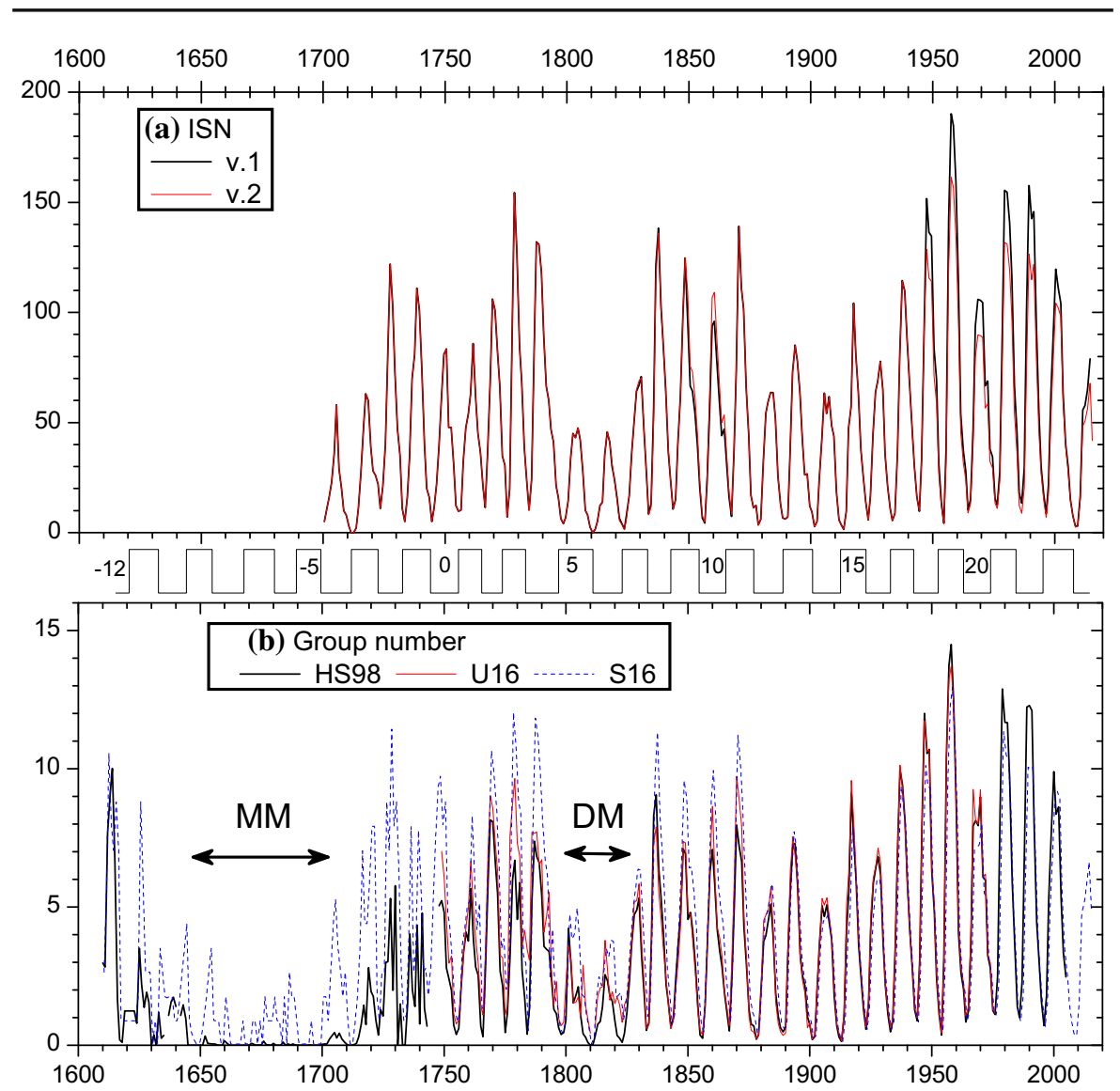

Fig. 1 Annual sunspot activity for the last centuries. a International sunspot number series versions 1 and 2 (the latter is scaled with a 0.6 factor, see SILSO, http://sidc.be/silso/datafiles). b Number of sunspot groups: HS98-(Hoyt and Schatten 1998); U16-(Usoskin et al. 2016b); S16-(Svalgaard and Schatten 2016). Standard (Zürich) cycle numbering is shown between the panels. Approximate dates of the Maunder minimum (MM) and Dalton minimum (DM) are shown in the lower panel

almost the same technique. This part of the series is fairly stable and homogeneous although an offset due to the change of the weighting procedure might have been introduced in 1945-1946 (Clette et al. 2014) but the correction for this effect is not clear and leads to uncertainties (Lockwood et al. 2014a; Friedli 2016). However, prior to that there have been many gaps in the data that were interpolated. If no sunspot observations are available for some period, the data gap is filled, without note in the final WSN series, using an interpolation between the available data and by employing some proxy data. In addition, earlier parts of the sunspot series were "corrected" by Wolf using geomagnetic observation (see details in Svalgaard 2012), which makes the series less homogeneous. Therefore, the WSN series is a combination of direct observations and interpolations for the period before 1849, leading to possible errors and inhomogeneities as discussed, e.g, by Vitinsky et al. (1986), Wilson (1998), Letfus (1999), Svalgaard (2012), Clette et al. (2014). The quality of the Wolf series before 
1749 is rather poor and hardly reliable (Hoyt et al. 1994; Hoyt and Schatten 1998; Hathaway and Wilson 2004).

The main problem of the WSN was a lack of documentation so that only the final product was available without information of the raw data, that made a full revision of the series hardly possible. Although this information does exist, it was hidden in hand-written notes of Rudolf Wolf and his successors. The situation is being improved now with an effort of the Rudolf Wolf Gesellschaft (http://www.wolfinstitute.ch) to scan and digitize the original Wolf's notes (Friedli 2016).

Note that the sun has been routinely photographed since 1876 so that full information on daily sunspot activity is available (the Greenwich series) for the last 140 years.

The routine production of the WSN series was terminated in Zürich in 1982. Since then, the sunspot number series is routinely updated as the International sunspot number (ISN) $R_{i}$, provided by the Solar Influences Data Analysis Center in Belgium (Clette et al. 2007). The international sunspot number series is computed using the same definition (Eq. 1) as WSN but it has a significant distinction from the WSN: it is based not on a single primary solar observation for each day but instead uses a weighted average of more than 20 approved observers. The ISN (see SILSO, http://sidc.be/ silso/datafiles) has been recently updated to version 2 with corrections to some known inhomogeneities (Clette et al. 2014). A potential user should know that the ISN (v.2) is calibrated to Wolfer, in contrast to earlier WSN and ISN (v.1) calibrated to Wolf. As a result, a constant scaling factor 0.6 should be applied to compare ISN (v.2) to ISN (v.1). The two versions are shown in Fig. 1a. One can see that the ISN v.2 (scaled by the 0.6 factor) is somewhat smaller than v. 1 after the 1940s, because of the correction for the Waldmeier discontinuity (see below), but they are nearly identical before that.

In addition to the standard sunspot number $R_{i}$, there is also a series of hemispheric sunspot numbers $R_{\mathrm{N}}$ and $R_{\mathrm{S}}$, which account for spots only in the northern and southern solar hemispheres, respectively (note that $R_{i}=R_{\mathrm{N}}+R_{\mathrm{S}}$ ). These series are used to study the N-S asymmetry of solar activity (Temmer et al. 2002).

\section{Group sunspot number (GSN) series}

Since the WSN series is of lower quality before the 1850s and is hardly reliable before 1750 , there was a need to re-evaluate early sunspot data. This tremendous work has been done by Hoyt and Schatten (1996, 1998), who performed an extensive archive search and nearly doubled the amount of original information compared to the Wolf series. They have produced a new series of sunspot activity called the group sunspot numbers (GSN_see Fig. 1b), including all available archival records. The daily group sunspot number $R_{g}$ is defined as follows:

$$
R_{g}=\frac{12.08}{n} \sum_{i} k_{i}^{\prime} G_{i}
$$

where $G_{i}$ is the number of sunspot groups recorded by the $i$-th observer, $k^{\prime}$ is the observer's individual correction factor, $n$ is the number of observers for the particular day, and 12.08 is a normalization number scaling $R_{g}$ to $R_{z}$ values for the period of 
1874-1976. However, the exact scaling factor 12.08 has recently been questioned due to an inhomogeneity within the RGO data between 1874-1885 (Cliver and Ling 2016; Willis et al. 2016). $R_{g}$ is more robust than $R_{z}$ or $R_{i}$ since it is based on more easily identified sunspot groups and does not include the number of individual spots. By this the GSN avoids a problem related to the visibility of small sunspots since a group of several small spots would appear as one blurred spot for an observer with a low-quality telescope. Another potential uncertainty may be related to the way of grouping individual spots into sunspot groups that could be done differently in the past and nowadays (Clette et al. 2014). This uncertainty directly affecting the GSN is also important for WSN/ISN series since the number of groups composes $50-90 \%$ of the WSN/ISN values.

Another important advantage of the GSN series is that all the raw data are available. The GSN series includes not only one "primary" observation, but all available observations, and covers the period since 1610, being, thus, 140 years longer than the original WSN series. It is particularly interesting that the period of the Maunder minimum (1645-1715) was surprisingly well covered with daily observations (Ribes and Nesme-Ribes 1993; Hoyt and Schatten 1996) allowing for a detailed analysis of sunspot activity during this grand minimum (see also Sect. 4.2). Systematic uncertainties of the $R_{g}$ values are estimated to be about $10 \%$ before 1640, less than 5\% from 1640-1728 and from 1800-1849, 15-20\% from 1728-1799, and about $1 \%$ since 1849 (Hoyt and Schatten 1998). The GSN series is more reliable and homogeneous than the WSN series before 1849. The two series are nearly identical after the 1870s (Hoyt and Schatten 1998; Letfus 1999; Hathaway and Wilson 2004). However, the GSN series still contains some lacunas, uncertainties and possible inhomogeneities (see, e.g.,Letfus 2000; Usoskin et al. 2003a; Vaquero et al. 2012; Cliver and Ling 2016).

\section{Updates of the series}

The search for other lost or missing records of past solar instrumental observations has not ended even since the extensive work by Hoyt and Schatten. Archival searches still give new interesting findings of forgotten sunspot observations, often outside major observatories - see a detailed review book by Vaquero and Vázquez (2009) and original papers by Casas et al. (2006), Vaquero et al. (2005, 2007), Arlt (2008), Arlt (2009). Interestingly, not only sunspot counts but also regular drawings, forgotten for centuries, are being restored nowadays in dusty archives. A very interesting work has been done by Rainer Arlt (Arlt 2008, 2009; Arlt and Abdolvand 2011; Arlt et al. 2013) on recovering, digitizing, and analyzing regular drawings by S.H. Schwabe of 1825-1867 and J.C. Staudacher of 1749-1796. This work led to the extension of the Maunder butterfly diagram for several solar cycles backwards (Arlt 2009; Usoskin et al. 2009c; Arlt and Abdolvand 2011; Arlt et al. 2013) — see a newly built butterfly diagram for solar cycles Nos. 7-10 shown in Fig. 2. A recent finding of the lost data by G. Marcgraf and correcting some earlier uncertain data for the period 1636-1642 by Vaquero et al. (2011) made it possible to revise the pattern of the beginning of the Maunder minimum. Recent corrections to the group number database have been collected (http://haso.unex.es/?q=content/data) by Vaquero et al. (2016) who updated the database of Hoyt and Schatten (1998) by correcting some errors and inexactitudes. 


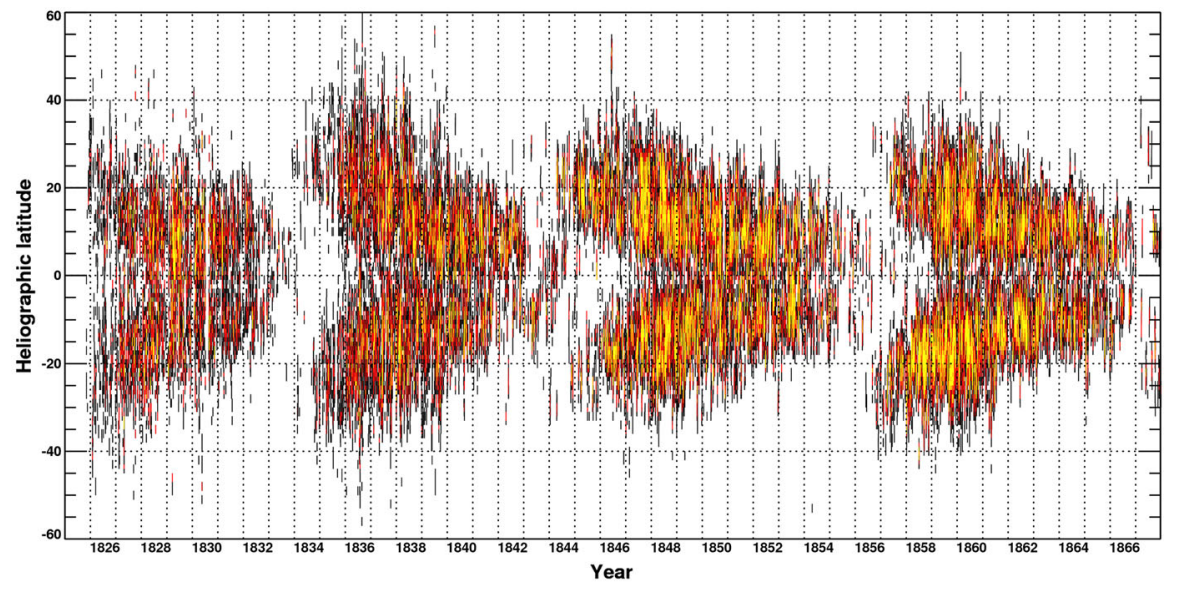

Fig. 2 Maunder butterfly diagram of sunspot occurrence reconstructed by Arlt et al. (2013) for 1825-1867 using recovered drawing of S.H. Schwabe

Several inconsistencies and discontinuities have been found in the existing sunspot series. For instance, Leussu et al. (2013) have shown that the values of WSN before 1848 (when Wolf had started his own observations) were overestimated by $\approx 20 \%$ because of the incorrect $k$-factor ascribed by Wolf to Schwabe. This error, called the "Wolf discontinuity", erroneously alters the WSN/ISN series but does not affect the GSN series. Another reported error is the so-called "Waldmeier discontinuity" around 1947 (Clette et al. 2014), related to the fact that Waldmeier had modified the procedure of counting spots, including 'weighting' sunspot number, without a proper noticing which led to a greater sunspot number compared to the standard technique. This suggests that the WSN/ISN may be overestimated by 10-20\% past 1947 (Clette et al. 2014; Lockwood et al. 2014b), but this does not directly affect the GSN series. As studied by Friedli (2016), this weighting might have been introduced intermittently already in the early twentieth century.

Clette et al. (2014) and Cliver and Ling (2016) proposed, based on the ratio of number of groups reported by Wolfer to that based on the Royal Greenwich Observatory (RGO) data, that there might be a transition in the calibration of GSN around the turn of the nineteenth to twentieth century (or even until 1915) related to inhomogeneous quality of the RGO data used to build the GSN at that period. On the other hand, independent observations of David Hadden from Iowa or Madrid Observatory (Carrasco et al. 2013; Aparicio et al. 2014) reveal that the problem with RGO data is essential only before the 1880s but not after that. Willis et al. (2016) studied RGO data for the years 1874-1885 and found that the database of Hoyt and Schatten (1998) might have slightly underestimated the RGO number of groups during that time. Sarychev and Roshchina (2009) suggested that the RGO data are erroneous for the period 18741880 but quite homogeneous after that. Vaquero et al. (2014) reported that ISN values for the period prior to 1850 are discordant with the number of spotless days, and concluded that the problem could be related to the calibration constants by Wolf (as found 
by Leussu et al. 2013) and to the non-linearity of ISN for low values. Most of these errors affect the WSN/ISN series, while GSN is more robust.

Thus, such inconsistencies should be investigated, and new series, with corrections of the known problems, need to be produced. Several such efforts have been made recently leading to inconsistent solar activity reconstructions. One of the new reconstructions was made by Clette et al. (2014) who introduced a revised version of the ISN (v.2 - see Fig. 1a), correcting the two apparent discontinuities, Wolf and Waldmeier, as described above. In addition, the entire series was rescaled to the reference level of Wolfer, while the 'classic' WSN/ISN series was scaled to Wolf. This leads to a constant scaling with the factor $1.667=1 / 6$ of the ISN_v.2 series with respect to other series. Keeping this scaling in mind, the ISN_v2 series is systematically different from the earlier one after the 1940s, and for a few decades in the mid-nineteenth century. This was not a fundamental revision by a scaling correction for a couple of errors.

A full revision of the GSN series was performed by Svalgaard and Schatten (2016) who used the number of sunspot group by Hoyt and Schatten (1998) but applied different method to construct the new GSN series. They also used a daisychain linear regression to calibrate different observers but doing it in several steps. A few key observers, called the 'backbones', were selected, and other observers were re-normalized to the 'backbones' using linear regressions. Then the 'backbones' were calibrated to each other, again using the linear scaling. Before 1800, when the daisy-chain calibration cannot be directly applied, two other methods, the 'high-low' (observers reporting larger number of groups were favored over those reporting smaller number of groups) and 'brightest star' (only the highest daily number of sunspot groups per year was considered) methods. This GSN series, called S16, is shown in Fig. 1b as the blue dotted curve. It suggests a much higher, than usually thought, level of solar activity in the 19th and especially 18th centuries, comparable to that during the mid-twentieth century. As a result of the 'brightest star' method, it yields moderate values during the Maunder minimum in contrast to the present paradigm of virtually no sunspots (Eddy 1976; Ribes and Nesme-Ribes 1993; Hoyt and Schatten 1996; Usoskin et al. 2015).

The use of the traditional $k$-factor method of linear scaling for inter-calibration of solar observers has been found invalid (Dudok et al. 2016; Lockwood et al. 2016c, b; Usoskin et al. 2016b), and a need for a modern non-parametric method has emerged. This is illustrated by Fig. 3 which shows the ratio of the sunspot group number reported by Wolfer, $G_{\text {Wolfer }}$, to that by Wolf, $G_{\text {Wolf }}$, for days when both reports are available. This ratio obviously depends on the level of activity, being about two for low-activity days $G_{\text {Wolfer }}=1$ and only $\approx 1.2$ for high-activity days. The ratio is strongly non-linear due to the fact that large sunspot groups dominate during periods of high activity. The horizontal dash-dotted line denotes the constant scaling $k$-factor of 1.667 used earlier (Clette et al. 2014) between Wolf and Wolfer. One can see that the use of the $k$-factor leads to a significant, by $\approx 40 \%$, over-correction of the numbers from Wolf when scaling them to Wolfer.

Several new methods, free of the linear assumption, have been proposed recently. One, called the ADF-method (Usoskin et al. 2016b), is based on a comparison of statistics of the active-day fraction (ADF) in the sunspot (group) records of an observer with that of the reference data-set (the RGO record of sunspot groups for the period 


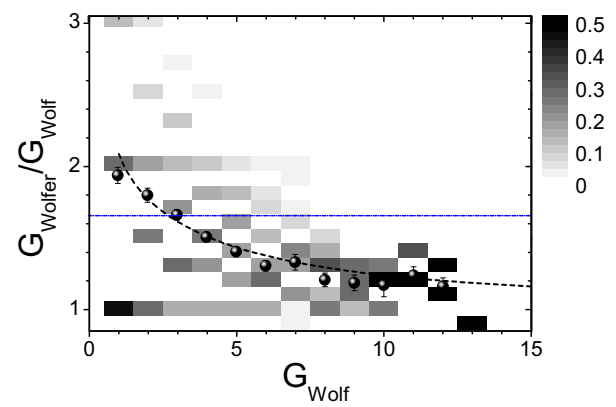

Fig. 3 Correction $c$-factor of Wolf to Wolfer. The grey scale represents the probability density function (PDF) of the ratio of the number of groups reported by Wolfer for days, when Wolf reported a given number of groups. The big dots with error bars depict the mean values. The dashed line is a functional exponential fit. The horizontal dot-dashed line represents the constant correction $k$-factor 1.667 (Clette et al. 2014). Modified after (Usoskin et al. 2016b)

1900-1976). By comparing them, the observational acuity threshold $A_{\text {th }}$ can be found defined so that the observer is supposed to report all the sunspot groups with area greater than the threshold and to miss smaller groups. This threshold characterizes the quality of the observer and is further used to calibrate his/her records. The values of the defined thresholds for some principal observers of the eighteenth to nineteenth century are given in Table 1. Based on the defined observational acuity thresholds for each observer, a new GSN series was constructed, called U16, is depicted in Fig. 1b as the red curve. It lies lower than GSN S16 around solar maxima but slightly higher than HS98, in the eighteenth-nineteenth centuries.

Another new method was proposed by Friedli (2016) who revised the WSN series basing on recently digitized Wolf's original notes and using the relation between numbers of groups and individual spots. This series appears consistent with the GSN Us16 series and close to the classical ISN series but is lower than the GSN S16 series.

Several attempts to test/validate different sunspot reconstructions using indirect proxies yielded indicative results: tests based on cosmogenic radionuclides (Asvestari et al. 2017), including ${ }^{44} \mathrm{Ti}$ in measured in the fallen meteorites (see Fig. 19) as well as geomagnetic and heliospheric proxies (Lockwood et al. 2016a, b) favor the 'lower' reconstructions (Hoyt and Schatten 1998; Usoskin et al. 2016b) against the 'high' reconstructions (Svalgaard and Schatten 2016). On the other hand, comparison with the solar open magnetic field models (Owens et al. 2016) cannot distinguish between different series.

The current situation with the sunspot number series is developing quickly and can hardly be resolved now. The old 'classical' WSN and GSN series need to be corrected for apparent inhomogeneities. Yet, newly emerging revisions of the sunspot series are mutually inconsistent and require efforts of the solar community on a consensus approach. On the other hand, the scientific community needs a 'consensus' series of solar activity, and the work in this direction is under way. Currently, probably the most reliable information of solar activity before the twentieth century can be obtained from cosmogenic isotope data (Sect. 3). This review will be updated as the situation progresses. 
Table 1 Values of the observational acuity threshold for the key observers of the 18-19th centuries (modified after Usoskin et al. 2016b) a The observational threshold is likely overestimated

Columns are: Name of the observer; Period of observation $T_{\text {obs }}$; Number of observational days $N$; The threshold area $A_{\text {th }}$ in uncorrected msd; values in parentheses denote the upper and lower $1 \sigma$ bound

\begin{tabular}{|c|c|c|c|}
\hline Observer & $T_{\mathrm{obs}}$ & $N$ & $S A_{\mathrm{th}}$ \\
\hline Quimby & 1889-1921 & 10830 & $22\left(\begin{array}{l}28 \\
16\end{array}\right)$ \\
\hline Wolfer & 1876-1928 & 7165 & $6\left(\begin{array}{c}12 \\
0\end{array}\right)$ \\
\hline Winkler $^{a}$ & 1882-1910 & 4812 & $53\left(\begin{array}{l}66 \\
45\end{array}\right)$ \\
\hline Tacchini & 1871-1900 & 6235 & $10\left(\begin{array}{c}14 \\
7\end{array}\right)$ \\
\hline Leppig & 1867-1881 & 2463 & $45\left(\begin{array}{l}33 \\
55\end{array}\right)$ \\
\hline Spörer & 1861-1893 & 5386 & $3\left(\begin{array}{l}5 \\
0\end{array}\right)$ \\
\hline Weber & 1859-1883 & 6981 & $22\left(\begin{array}{l}28 \\
16\end{array}\right)$ \\
\hline Wolf & $1848-1893$ & 8102 & $45\left(\begin{array}{l}53 \\
36\end{array}\right)$ \\
\hline Shea & $1847-1866$ & 5538 & $25\left(\begin{array}{l}33 \\
18\end{array}\right)$ \\
\hline Schmidt & $1841-1883$ & 6887 & $10\left(\begin{array}{c}15 \\
6\end{array}\right)$ \\
\hline Schwabe & $1825-1867$ & 8570 & $13\left(\begin{array}{c}18 \\
8\end{array}\right)$ \\
\hline Pastorff & 1819-1833 & 1451 & $5\left(\begin{array}{l}10 \\
0\end{array}\right)$ \\
\hline $\operatorname{Stark}^{a}$ & 1813-1836 & 2406 & $60\left(\begin{array}{l}70 \\
50\end{array}\right)$ \\
\hline Derfflinger $^{a}$ & $1802-1824$ & 346 & $50\left(\begin{array}{l}80 \\
40\end{array}\right)$ \\
\hline Herschel $^{a}$ & 1794-1818 & 344 & $23\left(\begin{array}{l}35 \\
10\end{array}\right)$ \\
\hline Horrebow & $1761-1776$ & 1365 & $75\left(\begin{array}{l}95 \\
60\end{array}\right)$ \\
\hline Schubert & $1754-1758$ & 404 & $10\left(\begin{array}{c}16 \\
5\end{array}\right)$ \\
\hline
\end{tabular}

\section{Other indices}

An example of a synthetic index of solar activity is the flare index, representing solar flare activity (e.g., Özgüç et al. 2003; Kleczek 1952). The flare index quantifies daily flare activity in the following manner; it is computed as a product of the flare's relative importance $I$ in the $\mathrm{H}_{\alpha}$-range and duration $t, Q=I t$, thus being a rough measure of the total energy emitted by the flare. The daily flare index is produced by Bogazici University (Özgüç et al. 2003) and is available since 1936.

A traditional physical index of solar activity is related to the radioflux of the sun in the wavelength range of $10.7 \mathrm{~cm}$ and is called the F10.7 index (e.g., Tapping and Charrois 1994). This index represents the flux (in solar flux units, $1 \mathrm{sfu}=$ $10^{-22} \mathrm{Wm}^{-2} \mathrm{~Hz}^{-1}$ ) of solar radio emission at a centimetric wavelength. There are at least two sources of $10.7 \mathrm{~cm}$ flux-free-free emission from hot coronal plasma and gyromagnetic emission from active regions (Tapping 1987). It is a good quantitative measure of the level of solar activity, which is not directly related to sunspots. Close correlation between the F10.7 index and sunspot number indicates that the latter is a 
good index of general solar activity, including coronal activity. The solar F10.7 cm record has been measured continuously since 1947.

Another physical index is the coronal index (e.g., Rybanský et al. 2005), which is a measure of the irradiance of the sun as a star in the coronal green line. Computation of the coronal index is based on observations of green corona intensities (Fe XIV emission line at $530.3 \mathrm{~nm}$ wavelength) from coronal stations all over the world, the data being transformed to the Lomnický Štit photometric scale. This index is considered a basic optical index of solar activity. A synthesized homogeneous database of the Fe XIV $530.3 \mathrm{~nm}$ coronal-emission line intensities has existed since 1943 and covers seven solar cycles.

Often sunspot area is considered as a physical index representing solar activity (e.g., Baranyi et al. 2001; Balmaceda et al. 2005). This index gives the total area of visible spots on the solar disc in units of millionths of the sun's visible hemisphere, corrected for apparent distortion due to the curvature of the solar surface. The area of individual groups may vary between tens of millionths (for small groups) up to several thousands of millionths for huge groups. This index has a physical meaning related to the solar magnetic flux emerging at sunspots. Sunspot areas are available since 1874 in the Greenwich series obtained from daily photographic images of the sun.

Sunspot group areas were routinely produced by the Royal Greenwich Observatory from daily photographic images of the sun for the period between 1874-1976 and after 1976 extended by the SOON network. Note, that the quality of the RGO data before 1880-1890s may be uneven (see discussion in Sect. 2.2.1). Sunspot areas can be reconstructed even before that using drawing of the sun by $\mathrm{H}$. Schwabe for the years 1826-1867 (Arlt et al. 2013; Senthamizh Pavai et al. 2015) and images by Spörer for the period 1861-1894 (Diercke et al. 2015). In addition, some fragmentary solar drawings exist even for earlier periods, including the Maunder minimum in the seventeenth century (Ribes and Nesme-Ribes 1993; Vaquero et al. 2004; Arlt 2008).

An important quantity is solar irradiance, total and spectral (Fröhlich 2012). Irradiance variations are physically related to solar magnetic variability (e.g., Solanki et al. 2000), and are often considered manifestations of solar activity, which is of primary importance for solar-terrestrial relations.

Other physical indices include spectral sun-as-star observations, such as the CaII-K index (e.g., Donnelly et al. 1994; Foukal 1996), the space-based Mg II core-to-wing ratio as an index of solar UVI (e.g., Donnelly et al. 1994; Viereck and Puga 1999; Snow et al. 2005) and many others.

All the above indices are closely correlated to sunspot numbers on the solar-cycle scale, but may depict quite different behavior on short or long timescales.

\subsubsection{Indirect indices}

Sometimes quantitative measures of solar-variability effects are also considered as indices of solar activity. These are related not to solar activity per se, but rather to its effect on different environments. Accordingly, such indices are called indirect, and can be roughly divided into terrestrial/geomagnetic and heliospheric/interplanetary.

Geomagnetic indices quantify different effects of geomagnetic activity ultimately caused by solar variability, mostly by variations of solar-wind properties and the inter- 
planetary magnetic field. For example, the $a a$-index, which provides a global index of magnetic activity relative to a quiet-day curve for a pair of antipodal magnetic observatories (in England and Australia), is available from 1868 (Mayaud 1972). An extension of the geomagnetic series is available from the 1840s using the Helsinki $A k(H)$ index (Nevanlinna 2004a, b). Although the homogeneity of the geomagnetic series is compromised (e.g., Lukianova et al. 2009; Love 2011), it still remains an important indirect index of solar activity. A review of the geomagnetic effects of solar activity can be found, e.g., in Pulkkinen (2007). It is noteworthy that geomagnetic indices, in particular low-latitude aurorae (Silverman 2006), are associated with coronal/interplanetary activity (high-speed solar-wind streams, interplanetary transients, etc.) that may not be directly related to the sunspot-cycle phase and amplitude, and therefore serve only as an approximate index of solar activity. One of the earliest instrumental geomagnetic indices is related to the daily magnetic declination range, the range of diurnal variation of magnetic needle readings at a fixed location, and is available from the 1780s (Nevanlinna 1995). However, this data exists as several fragmentary sets, which are difficult to combine into a homogeneous data series.

Several geomagnetic activity indices have been proposed recently. One is the IDV (interdiurnal variability) index (Svalgaard and Cliver 2005; Lockwood et al. 2013) based on Bartels' historical $u$-index of geomagnetic activity, related to the difference between successive daily values of the horizontal or vertical component of the geomagnetic field. Another index is IHV (inter-hourly variability) calculated from the absolute differences between successive hourly values of the horizontal component of the geomagnetic field during night hours to minimize the effect of the daily curve (Svalgaard et al. 2004; Mursula and Martini 2006). Some details of the derivation and use of these indirect indices for long-term solar-activity studies can be found, e.g., in the Living Review by Lockwood (2013).

Heliospheric indices are related to features of the solar wind or the interplanetary magnetic field measured (or estimated) in the interplanetary space. For example, the time evolution of the total (or open) solar magnetic flux is extensively debated (e.g., Lockwood et al. 1999; Wang et al. 2005; Krivova et al. 2007).

A special case of heliospheric indices is related to the galactic cosmic-ray intensity recorded in natural terrestrial archives. Since this indirect proxy is based on data recorded naturally throughout the ages and revealed now, it makes possible the reconstruction of solar activity changes on long timescales, as discussed in Sect. 3.

\subsection{Solar activity observations in the pre-telescopic epoch}

Instrumental solar data is based on regular observation (drawings or counting of spots) of the sun using optical instruments, e.g., the telescope used by Galileo in the early seventeenth century. These observations have mostly been made by professional astronomers whose qualifications and scientific thoroughness were doubtless. They form the basis of the group sunspot number series (Hoyt and Schatten 1998), which can be more-or-less reliably extended back to 1610 (see discussion in Sect. 2.2.1). However, some fragmentary records of qualitative solar and geomagnetic observations exist even for earlier times, as discussed below (Sects. 2.3.1-2.3.2). 


\subsubsection{Instrumental observations: camera obscura}

The invention of the telescope revolutionized astronomy. However, another solar astronomical instrument, the camera obscura, also made it possible to provide relatively good solar images and was still in use until the late eighteenth century. Camera obscuras were known from early times, and they have been used in major cathedrals to define the sun's position (see the review by Vaquero 2007; Vaquero and Vázquez 2009). The earliest known drawing of the solar disc was made by Frisius, who observed the solar eclipse in 1544 using a camera obscura. That observation was performed during the Spörer minimum and no spots were observed on the sun. The first known observation of a sunspot using a camera obscura was done by Kepler in May 1607, who erroneously ascribed the spot on the sun to a transit of Mercury. Although such observations were sparse and related to other phenomena (solar eclipses or transits of planets), there were also regular solar observations by camera obscura. For example, about 300 pages of logs of solar observations made in the cathedral of San Petronio in Bologna from 1655-1736 were published by Eustachio Manfredi in 1736 (see the full story in Vaquero 2007). Therefore, observations and drawings made using camera obscura can be regarded as instrumental observations.

\subsubsection{Naked-eye observations}

Even before regular professional observations performed with the aid of speciallydeveloped instruments (what we now regard as scientific observations) people were interested in unusual phenomena. Several historical records exist based on naked-eye observations of transient phenomena on the sun or in the sky.

From even before the telescopic era, a large amount of evidence of spots being observed on the solar disc can be traced back as far as to the middle of the fourth century BC (Theophrastus of Athens). The earliest known drawing of sunspots is dated to December 8, 1128 AD as published in "The Chronicle of John of Worcester" (Willis and Stephenson 2001). However, such evidence from Occidental and Moslem sources is scarce and mostly related to observations of transits of inner planets over the sun's disc, probably because of the dominance of the dogma on the perfectness of the sun's body, which dates back to Aristotle's doctrine (Bray and Loughhead 1964). Oriental sources are much richer for naked-eye sunspot records, but that data is also fragmentary and irregular (see, e.g., Clark and Stephenson 1978; Wittmann and Xu 1987; Yau and Stephenson 1988). Spots on the sun are mentioned in official Chinese and Korean chronicles from $165 \mathrm{BC}$ to $1918 \mathrm{AD}$. While these chronicles are fairly reliable, the data is not straightforward to interpret since it can be influenced by meteorological phenomena, e.g., dust loading in the atmosphere due to dust storms (Willis et al. 1980) or volcanic eruptions (Scuderi 1990) can facilitate sunspots observations. Direct comparison of Oriental naked-eye sunspot observations and European telescopic data shows that naked-eye observations can serve only as a qualitative indicator of sunspot activity, but can hardly be quantitatively interpreted (see, e.g., Willis et al. 1996, and references therein). Moreover, as a modern experiment of naked-eye observations (Mossman 1989) shows, Oriental chronicles contain only a tiny $(1 / 200-1 / 1000)$ fraction of the number of sunspots potentially visible with the naked eye (Eddy et al. 
1989). This indicates that records of sunspot observations in the official chronicles were highly irregular (Eddy 1983) and probably dependent on dominating traditions during specific historical periods (Clark and Stephenson 1978). Although naked-eye observations tend to qualitatively follow the general trend in solar activity according to a posteriori information (e.g., Vaquero et al. 2002), extraction of any independent quantitative information from these records seems impossible.

Visual observations of aurorae borealis at middle latitudes form another proxy for solar activity (e.g., Siscoe 1980; Schove 1983; Křivský 1984; Silverman 1992; Schröder 1992; Lee et al. 2004; Basurah 2004; Vázquez and Vaquero 2010). Fragmentary records of aurorae can be found in both Occidental and Oriental sources since antiquity. The first known dated notation of an aurora is from March 12, $567 \mathrm{BC}$ from Babylon (Stephenson et al. 2004). Aurorae may appear at middle latitudes as a result of enhanced geomagnetic activity due to transient interplanetary phenomena. Although auroral activity reflects coronal and interplanetary features rather than magnetic fields on the solar surface, there is a strong correlation between long-term variations of sunspot numbers and the frequency and latitude extent of aurora occurrences. Because of the phenomenon's short duration and low brightness, the probability of seeing aurora is severely affected by other factors such as the weather (sky overcast, heat lightnings), the Moon's phase, season, etc. The fact that these observations were not systematic in early times (before the beginning of the eighteenth century) makes it difficult to produce a homogeneous data set. Moreover, the geomagnetic latitude of the same geographical location may change quite dramatically over centuries, due to the migration of the geomagnetic axis, which also affects the probability of watching aurorae (Siscoe and Verosub 1983; Oguti and Egeland 1995). For example, the geomagnetic latitude of Seoul $\left(37.5^{\circ} \mathrm{N} 127^{\circ} \mathrm{E}\right)$, which is currently less than $30^{\circ}$, was about $40^{\circ}$ a millennium ago (Kovaltsov and Usoskin 2007). This dramatic change alone can explain the enhanced frequency of aurorae observations recorded in oriental chronicles.

\subsubsection{Mathematical/statistical extrapolations}

Due to the lack of reliable information regarding solar activity in the pre-instrumental era, it seems natural to try to extend the sunspot series back in time, before 1610 AD, by means of extrapolating its statistical properties. Indeed, numerous attempts of this kind have been made even recently (e.g., Nagovitsyn 1997; de Meyer 1998; Rigozo et al. 2001; Zharkova et al. 2015). Such models aim to find the main feature of the actually-observed sunspot series, e.g., a modulated carrier frequency or a multi-harmonic representation, which is then extrapolated backwards in time. The main disadvantage of this approach is that it is not a reconstruction based upon measured or observed quantities, but rather a "post-diction" based on extrapolation. This method is often used for short-term predictions, but it can hardly be used for the reliable long-term reconstruction of solar activity. In particular, it assumes that the sunspot time series is stationary, i.e., a limited time realization contains full information on its future and past. Clearly, such models cannot include periods exceeding the time span of observations upon which the extrapolation is based. Hence, the pre- or post-diction becomes increasingly unreliable with growing extrapolation time and its accuracy is hard to estimate. 
Sometimes a combination of the above approaches is used, i.e., a fit of the mathematical model to indirect qualitative proxy data. In such models a mathematical extrapolation of the sunspot series is slightly tuned and fitted to some proxy data for earlier times. For example, Schove $(1955,1979)$ fitted the slightly variable but phaselocked carrier frequency (about 11 years) to fragmentary data from naked-eye sunspot observations and auroral sightings. The phase locking is achieved by assuming exactly nine solar cycles per calendar century. This series, known as Schove series, reflects qualitative long-term variations of the solar activity, including some grand minima, but cannot pretend to be a quantitative representation in solar activity level. The Schove series played an important historical role in the 1960s. In particular, a comparison of the $\Delta^{14} \mathrm{C}$ data with this series succeeded in convincing the scientific community that secular variations of ${ }^{14} \mathrm{C}$ in tree rings have solar and not climatic origins (Stuiver 1961). This formed a cornerstone of the precise method of solar-activity reconstruction, which uses cosmogenic isotopes from terrestrial archives. However, attempts to reconstruct the phase and amplitude of the 11-year cycle, using this method, were unsuccessful. For example, Schove (1955) made predictions of forthcoming solar cycles up to 2005 , which failed. We note that all these works are not able to reproduce, for example, the Maunder minimum (which cannot be represented as a result of the superposition of different harmonic oscillations), yielding too high sunspot activity compared to that observed. From the modern point of view, the Schove series can be regarded as archaic.

\subsection{The solar cycle and its variations}

\subsubsection{Quasi-periodicities}

\section{1-years Schwabe cycle}

The main feature of solar activity is its pronounced quasi-periodicity with a period of about 11 years, known as the Schwabe cycle. However, the cycle varies in both amplitude and duration. The first observation of a possible regular variability in sunspot numbers was made by the Danish astronomer Christian Horrebow in the 1770s on the basis of his sunspot observations from 1761-1769 (see details in Gleissberg 1952; Vitinsky 1965), but the results were forgotten. It took over 70 years before the amateur astronomer Schwabe announced in 1844 that sunspot activity varies cyclically with a period of about 10 years. This cycle, called the 11-year or Schwabe cycle, is the most prominent variability in the sunspot-number series. It is recognized now as a fundamental feature of solar activity originating from the solar-dynamo process. This 11-year cyclicity is prominent in many other parameters including solar, heliospheric, geomagnetic, space weather, climate and others. The background for the 11-year Schwabe cycle is the 22-year Hale magnetic polarity cycle. Hale found that the polarity of sunspot magnetic fields changes in both hemispheres when a new 11-year cycle starts (Hale et al. 1919). This relates to the reversal of the global magnetic field of the sun with the period of 22 years. It is often considered that the 11-year Schwabe cycle is the modulo of the sign-alternating Hale cycle (e.g., Sonett 1983; Bracewell 1986; Kurths and Ruzmaikin 1990; de Meyer 1998; Mininni et al. 2001), but this is 
only a mathematical representation. A detailed review of solar cyclic variability can be found in (Hathaway 2015).

\section{Phase catastrophe?}

Sometimes the regular time evolution of solar activity is broken up by periods of greatly depressed activity called grand minima. The last grand minimum (and the only one covered by direct solar observations) was the famous Maunder minimum from 1645-1715 (Eddy 1976, 1983). Other grand minima in the past, known from cosmogenic isotope data, include, e.g., the Spörer minimum around 1450-1550 and the Wolf minimum around the fourteenth century (see the detailed discussion in Sect. 4.2). Sometimes the Dalton minimum (ca. 1790-1820) is also considered to be a grand minimum. However, sunspot activity was not completely suppressed and still showed Schwabe cyclicity during the Dalton minimum. As suggested by Schüssler et al. (1997), this can be a separate, intermediate state of the dynamo between the grand minimum and normal activity, or an unsuccessful attempt of the sun to switch to the grand minimum state (Frick et al. 1997; Sokoloff 2004). This is observed as the phase catastrophe of solar-activity evolution (e.g., Vitinsky et al. 1986; Kremliovsky 1994). A peculiarity in the phase evolution of sunspot activity around 1800 was also noted by Sonett (1983), who ascribed it to a possible error in Wolf sunspot data and by Wilson (1988a), who reported on a possible misplacement of sunspot minima for cycles 4-6 in the WSN series. It has been also suggested that the phase catastrophe can be related to a tiny cycle, which might have been lost at the end of the eighteenth century because of very sparse observations (Usoskin et al. 2001a, 2002b, 2003b; Zolotova and Ponyavin 2007). We note that a new independent evidence proving the existence of the lost cycle has been found recently in the reconstructed sunspot butterfly diagram for that period (Usoskin et al. 2009c).

\section{Centennial Gleissberg cycle}

The long-term change (trend) in the Schwabe cycle amplitude is known as the secular Gleissberg cycle (Gleissberg 1939) with the mean period of about 90 years. However, the Gleissberg cycle is not a cycle in the strict periodic sense but rather a modulation of the cycle envelope with a varying timescale of 60-120 years (e.g., Gleissberg 1971; Kuklin 1976; Ogurtsov et al. 2002).

Longer (super-secular) cycles cannot be studied using direct solar observations, but only indicatively by means of indirect proxies such as cosmogenic isotopes discussed in Sect. 3. Analysis of the proxy data also yields the Gleissberg secular cycle (Feynman and Gabriel 1990; Peristykh and Damon 2003), but the question of its phase locking and persistency/intermittency still remains open.

\section{0-years Suess/de Vries cycle}

Several longer cycles have been found in the cosmogenic isotope data. A cycle with a period of 205-210 years, called the de Vries or Suess cycle in different sources, is a prominent feature, observed in various cosmogenic data (e.g., Suess 1980; Sonett and 
Finney 1990; Zhentao 1990; Usoskin et al. 2004; Steinhilber et al. 2012). It mostly manifests itself as the recurrence period of Grand minima within clusters (Usoskin et al. 2007).

\section{Millennial Eddy cycle}

Sometimes variations with a characteristic time of 600-700 years or 1000-1200 years are discussed (e.g., Vitinsky et al. 1986; Sonett and Finney 1990; Vasiliev and Dergachev 2002; Steinhilber et al. 2012; Abreu et al. 2012), but they are intermittent and can hardly be regarded as a typical feature of solar activity. Sometimes it is called Eddy cycle (Steinhilber et al. 2012).

\section{$\approx 2400$-years Hallstatt cycle}

A 2000-2400-year cycle is also noticeable in radiocarbon data series (see, e.g., Vitinsky et al. 1986; Damon and Sonett 1991; Vasiliev and Dergachev 2002).

It can be studied only using very long series, covering the whole Holocene. It was traditionally ascribed to climatic or geomagnetic variability (Vasiliev and Dergachev 2002; Vasiliev et al. 2012) but a recent joint study (Usoskin et al. 2016a) of ${ }^{14} \mathrm{C}$ and ${ }^{10} \mathrm{Be}$ data-sets has shown that the Hallstatt cycle is of solar origin and is manifested through clustered occurrence of Grand minima and maxima around its lows and highs, respectively.

\subsubsection{Randomness versus regularity}

The short-term (days-months) variability of sunspot numbers is greater than the observational uncertainties indicating the presence of random fluctuations (noise). As typical for most real signals, this noise is not uniform (white), but rather red or correlated noise (e.g., Ostryakov and Usoskin 1990a; Oliver and Ballester 1996; Frick et al. 1997), namely, its variance depends on the level of the signal. While the existence of regularity and randomness in sunspot series is apparent, their relationship is not clear (e.g., Wilson 1994)—are they mutually independent or intrinsically tied together? Moreover, the question of whether randomness in sunspot data is due to chaotic or stochastic processes is still open.

Earlier it was common to describe sunspot activity as a multi-harmonic process with several basic harmonics (e.g., Vitinsky 1965; Sonett 1983; Vitinsky et al. 1986) with an addition of random noise, which plays no role in the solar-cycle evolution. However, it has been shown (e.g., Rozelot 1994; Weiss and Tobias 2000; Charbonneau 2001; Mininni et al. 2002) that such an oversimplified approach depends on the chosen reference time interval and does not adequately describe the long-term evolution of solar activity. A multi-harmonic representation is based on an assumption of the stationarity of the benchmark series, but this assumption is broadly invalid for solar activity (e.g., Kremliovsky 1994; Sello 2000; Polygiannakis et al. 2003). Moreover, a multi-harmonic representation cannot, for an apparent reason, be extrapolated to a timescale larger than that covered by the benchmark series. The fact that purely mathematical/statistical models cannot give good predictions of solar activity (as will 
be discussed later) implies that the nature of the solar cycle is not a multi-periodic or other purely deterministic process, but random (chaotic or stochastic) processes play an essential role in sunspot cycle formation (e.g., Moss et al. 2008; Käpylä et al. 2012). An old idea of the possible planetary influence on the dynamo has received a new pulse recently with some unspecified torque effect on the assumed quasi-rigid non-axisymmetric tahocline (Abreu et al. 2012). However, this result was criticized by Poluianov and Usoskin (2014) as being an artifact of an inappropriate analysis (aliasing effect of incorrect smoothing). In addition, Cauquoin et al. (2014) have shown that such periodicities were not observed in ${ }^{10} \mathrm{Be}$ data $330 \mathrm{kyr}$ ago.

Different numeric tests, such as an analysis of the Lyapunov exponents (Ostryakov and Usoskin 1990b; Mundt et al. 1991; Kremliovsky 1995; Sello 2000), Kolmogorov entropy (Carbonell et al. 1994; Sello 2000) and Hurst exponent (Ruzmaikin et al. 1994; Oliver and Ballester 1998), confirm the chaotic/stochastic nature of the solar-activity time evolution (see, e.g., a review by Panchev and Tsekov 2007).

It was suggested quite a while ago that the variability of the solar cycle may be a temporal realization of a low-dimensional chaotic system (e.g., Ruzmaikin 1981). This concept became popular in the early 1990s, when many authors considered solar activity as an example of low-dimensional deterministic chaos, described by the strange attractor (e.g., Kurths and Ruzmaikin 1990; Ostryakov and Usoskin 1990b; Morfill et al. 1991; Mundt et al. 1991; Rozelot 1995; Salakhutdinova 1999; Serre and NesmeRibes 2000; Hanslmeier et al. 2013). Such a process naturally contains randomness, which is an intrinsic feature of the system rather than an independent additive or multiplicative noise. However, although this approach easily produces features seemingly similar to those of solar activity, quantitative parameters of the low-dimensional attractor have varied greatly as obtained by different authors. Later it was realized that the analyzed data set was too short (Carbonell et al. 1993, 1994), and the results were strongly dependent on the choice of filtering methods (Price et al. 1992). Developing this approach, Mininni et al. (2000, 2001) suggest that one considers sunspot activity as an example of a 2D Van der Pol relaxation oscillator with an intrinsic stochastic component.

Such phenomenological or basic principles models, while succeeding in reproducing (to some extent) the observed features of solar-activity variability, do not provide insight into the nature of regular and random components of solar variability. In this sense efforts to understand the nature of randomness in sunspot activity in the framework of dynamo theory are more advanced. Corresponding theoretical dynamo models have been developed (see reviews by Ossendrijver 2003; Charbonneau 2010), which include stochastic processes (e.g., Weiss et al. 1984; Feynman and Gabriel 1990; Schmalz and Stix 1991; Moss et al. 1992; Hoyng 1993; Brooke and Moss 1994; Lawrence et al. 1995; Schmitt et al. 1996; Charbonneau and Dikpati 2000; Brandenburg and Sokoloff 2002). For example, Feynman and Gabriel (1990) suggest that the transition from a regular to a chaotic dynamo passes through bifurcation. Charbonneau and Dikpati (2000) studied stochastic fluctuations in a Babcock-Leighton dynamo model and succeeded in the qualitative reproduction of the anti-correlation between cycle amplitude and length (Waldmeier rule). Their model also predicts a phase-lock of the Schwabe cycle, i.e., that the 11-year cycle is an internal "clock" of the sun. Most often the idea of fluctuations is related to the $\alpha$-effect, which is the 
result of the electromotive force averaged over turbulent vortices, and thus can contain a fluctuating contribution (e.g., Hoyng 1993; Ossendrijver et al. 1996; Brandenburg and Spiegel 2008; Moss et al. 2008). Note that a significant fluctuating component (with the amplitude more than $100 \%$ of the regular component) is essential in all these models.

\subsubsection{A note on solar activity predictions}

Randomness (see Sect. 2.4.2) in the SN series is directly related to the predictability of solar activity. Forecasting solar activity has been a subject of intense study for many years (e.g., Yule 1927; Newton 1928; Gleissberg 1948; Vitinsky 1965) and has greatly intensified recently with a hundred of journal articles being published to predict the solar cycle No. 24 maximum (see, e.g., reviews by Pesnell 2012, 2016), following the boost of space-technology development and increasing debates on solar-terrestrial relations. In fact, the situation has not been improved since the previous cycle, No. 23. The predictions for the peak sunspot number of solar cycle No. 24 range by a factor of 5, between 40 and 200, reflecting the lack of a reliable consensus method (Tobias et al. 2006). Detailed review of the solar activity prediction methods and results have been recently provided by (Hathaway 2009; Petrovay 2010; Pesnell 2012).

A detailed classification of the prediction methods is given by Pesnell (2012) who separates climatology, precursor, theoretical (dynamo model), spectral, neural network, and stock market prediction methods. All prediction methods can be generically divided into precursor and statistical (including the majority of the above classifications) techniques or their combinations (Hathaway et al. 1999). The fact that the prediction of solar cycle is not improved with adding more data (the new solar cycle) suggests that such methods are not able to give reliable prognoses.

The precursor methods are usually based on phenomenological, but sometimes physical, links between the poloidal solar-magnetic field, estimated, e.g., from geomagnetic activity in the declining phase of the preceding cycle or in the minimum time (e.g.,Hathaway 2009), with the toroidal field responsible for sunspot formation. These methods usually yield better short-term predictions of a forthcoming cycle maximum than the statistical methods, but cannot be applied to timescales longer than one solar cycle.

Statistical methods, including a low-dimensional solar-attractor representation (Kurths and Ruzmaikin 1990), are based solely on the statistical properties of sunspot activity and may give a reasonable result for short-term forecasting, but yield very poor results for long-term predictions (see reviews by, e.g., Conway 1998; Hathaway et al. 1999; Li et al. 2001; Usoskin and Mursula 2003; Kane 2007) because of chaotic/stochastic behavior (see Sect. 2.4.2).

A new method based on sophisticated dynamo numerical simulations emerges (e.g., Dikpati and Gilman 2006; Dikpati et al. 2008; Choudhuri et al. 2007; Jiang et al. 2007), but the results are so far contradictory with each other. Prospectives of this approach are also not clear because of the stochastic component, which drives the dynamo out of the deterministic regime, and uncertainties in the input parameters (Tobias et al. 2006; Bushby and Tobias 2007; Karak and Nandy 2012). 
Some models, mostly based on precursor method, succeed in reasonable predictions of a forthcoming solar cycle (i.e., several years ahead), but they do not pretend to extend further in time. On the other hand, many claims of the solar activity forecast for 40-50 years ahead and even beyond that, even millennia ahead (Zharkova et al. 2015), have been made recently, often without sensible argumentation. However, so far there is no evidence of any method giving a reasonable prediction of solar activity beyond the solar-cycle scale (see, e.g., Sect. 2.3.3), probably because of the intrinsic limit of solar-activity predictability due to its stochastic/chaotic nature (Kremliovsky 1995; Tobias et al. 2006). Accordingly, such attempts can be regarded as speculative, unless they are verified by the actual behavior of solar activity. Note that even an exact prediction of the amplitude of one solar cycle can be just a random coincidence and cannot serve as a proof of the method's veracity. Only a sequence of successful predictions can form a basis for confidence, which requires several decades.

Note that several "predictions" of the general decline of the coming solar activity have been made recently (Solanki et al. 2004; Abreu et al. 2008; Lockwood et al. 2011), however, these are not really true predictions but rather acknowledgements of the fact that the Modern Grand maximum (Usoskin et al. 2003c; Solanki et al. 2004) has ceased. Similar caution can be made about predictions of a Grand minimum (e.g., Lockwood et al. 2011; Miyahara et al. 2010)_a grand minimum should appear soon or later, but presently we are hardly able to predict its occurrence.

\subsection{Summary}

In this section, the concept of solar activity and quantifying indices is discussed, as well as the main features of solar-activity temporal behavior.

The concept of solar activity is quite broad and covers non-stationary and nonequilibrium (often eruptive) processes, in contrast to the "quiet" sun concept, and their effects upon the terrestrial and heliospheric environment. Many indices are used to quantify different aspects of variable solar activity. Quantitative indices include direct (i.e., related directly to solar variability) and indirect (i.e., related to terrestrial and interplanetary effects caused by solar activity), they can be physical or synthetic. While all indices depict the dominant 11-year cyclic variability, their relationships on other timescales (short scale or long-term trends) may vary to a great extent.

The most common and the longest available index of solar activity is the sunspot number, which is a synthetic index and is useful for the quantitative representation of overall solar activity outside the grand minimum. During the grand Maunder minimum, however, it may give only a clue about solar activity whose level may drop below the sunspot formation threshold. The sunspot number series is available for the period from $1610 \mathrm{AD}$, after the invention of the telescope, and covers, in particular, the Maunder minimum in the late seventeenth century. However, this series has big uncertainties before 1900 (Sect. 2.2.1). Fragmentary non-instrumental observations of the sun before 1610, while giving a possible hint of relative changes in solar activity, cannot be interpreted in a quantitative manner.

Solar activity in all its manifestations is dominated by the 11-year Schwabe cycle, which has, in fact, a variable length of 9-14 years for individual cycles. The amplitude 
of the Schwabe cycle varies greatly-from the almost spotless Maunder minimum to the very high cycle 19, possibly in relation to the Gleissberg or secular cycle. Longer super-secular characteristic times can also be found in various proxies of solar activity, as discussed in Sect. 4.

Solar activity contains essential chaotic/stochastic components, that lead to irregular variations and make the prediction of solar activity for a timescale exceeding one solar cycle impossible.

\section{The proxy method of past solar-activity reconstruction}

In addition to direct solar observations, described in Sect. 2.2.1, there are also indirect solar proxies, which are used to study solar activity in the pre-telescopic era. Unfortunately, we do not have any reliable data that could give a direct index of solar variability before the beginning of the sunspot-number series. Therefore, one must use indirect proxies, i.e., quantitative parameters, which can be measured nowadays but represent different effects of solar magnetic activity in the past. It is common to use, for this purpose, signatures of terrestrial indirect effects induced by variable solar-magnetic activity, that is stored in natural archives. Such traceable signatures can be related to nuclear (used in the cosmogenic-isotope method) or chemical (used, e.g., in the nitrate method) effects caused by cosmic rays (CRs) in the Earth's atmosphere, lunar rocks or meteorites.

The most common proxy of solar activity is formed by the data on cosmogenic radionuclides (e.g., ${ }^{10} \mathrm{Be}$ and ${ }^{14} \mathrm{C}$ ), which are produced by cosmic rays in the Earth's atmosphere (e.g, Stuiver and Quay 1980; Beer et al. 1990; Bard et al. 1997; Beer 2000; Beer et al. 2012).

Other cosmogenic nuclides, which are used in geological and paleomagnetic dating, are less suitable for studies of solar activity (see e.g., Beer 2000; Beer et al. 2012). Cosmic rays are the main source of cosmogenic nuclides in the atmosphere (excluding anthropogenic factors during the last decades) with the maximum production being in the upper troposphere-lower stratosphere. After a complicated transport in the atmosphere, the cosmogenic isotopes are stored in natural archives such as polar ice, trees, marine sediments, etc. This process is also affected by changes in the geomagnetic field and climate. Cosmic rays experience heliospheric modulation due to solar wind and the frozen-in solar magnetic field. The intensity of modulation depends on solar activity and, therefore, cosmic-ray flux and the ensuing cosmogenic isotope intensity depends inversely on solar activity. An important advantage of the cosmogenic data is that primary archiving is done naturally in a similar manner throughout the ages, and these archives are measured nowadays in laboratories using modern techniques. If necessary, all measurements can be repeated and improved, as has been done for some radiocarbon samples. In contrast to fixed historical archival data (such as sunspot or auroral observations) this approach makes it possible to obtain homogeneous data sets of stable quality and to improve the quality of data with the invention of new methods (such as accelerator mass spectrometry). Cosmogenic isotope data is the main regular indicator of solar activity on the very long-term scale but it cannot resolve the details of individual solar cycles. The redistribution of nuclides in terrestrial reservoirs and 
archiving may be affected by local and global climate/circulation processes, which are, to a large extent, unknown for the past. However, a combined study of different nuclides data, whose responses to terrestrial effects are very different, may allow for disentangling external and terrestrial signals.

\subsection{The physical basis of the method}

\subsubsection{Heliospheric modulation of cosmic rays}

The flux of cosmic rays (highly energetic fully ionized nuclei) is considered roughly constant (at least at the time scales relevant for the present study) in the vicinity of the Solar system. However, before reaching the vicinity of Earth, galactic cosmic rays experience complicated transport in the heliosphere that leads to modulation of their flux. Heliospheric transport of GCR is described by Parker's theory (Parker 1965; Toptygin 1985) and includes four basic processes: the diffusion of particles due to their scattering on magnetic inhomogeneities, the convection of particles by out-blowing solar wind, adiabatic energy losses in expanding solar wind, drifts of particles in the magnetic field, including the gradient-curvature drift in the regular heliospheric magnetic field, and the drift along the heliospheric current sheet, which is a thin magnetic interface between the two heliomagnetic hemispheres (Potgieter 2013). Because of variable solar-magnetic activity, CR flux in the vicinity of Earth is strongly modulated (see Fig. 4). The most prominent feature in CR modulation is the 11-year cycle, which is in inverse relation to solar activity. The 11-year cycle in CR is delayed (from a month up to two years) with respect to the sunspots (Usoskin et al. 1998).
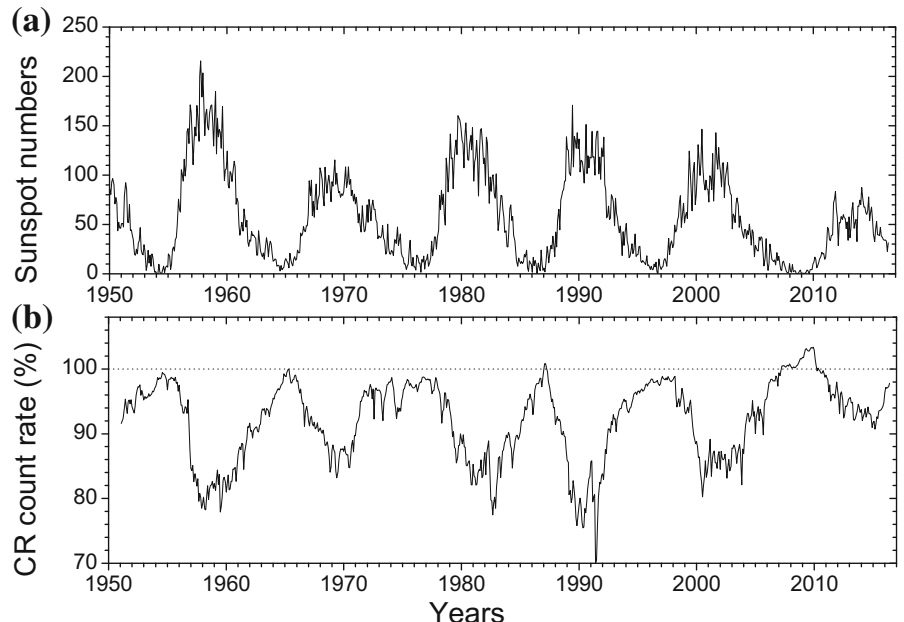

Fig. 4 Cyclic variations since 1951. a Time profiles of International sunspot number v.2 (scaled with 0.6factor) (http://sidc.be/silso/datafiles); b cosmic-ray flux as the count rate of a polar neutron monitor (Oulu NM http://cosmicrays.oulu.fi, Climax NM data used before 1964), 100\% NM count rate corresponds to May 1965 
The time profile of cosmic-ray flux as measured by a neutron monitor (NM) is shown in Fig. 4 (panel b) together with the sunspot numbers (panel a). Besides the inverse relation between them, some other features can also be noted. A 22-year cyclicity manifests itself in cosmic-ray modulation through the alteration of sharp and flat maxima in cosmic-ray data, originated from the charge-dependent drift mechanism. One may also note short-term fluctuations, which are not directly related to sunspot numbers but are driven by interplanetary transients caused by solar eruptive events, e.g., flares or CMEs. An interesting feature is related to the recent decade. The CR flux in 2009 was the highest ever recorded by NMs (Moraal and Stoker 2010), as caused by the favorable heliospheric conditions (unusually weak heliospheric magnetic field and the flat heliospheric current sheet) (McDonald et al. 2010). On the other hand, the sunspot minimum was comparable to other minima. The level of CR modulation during the cycle 24 was moderate, much more shallow than for the previous cycles, reflecting the weak solar cycle 24 . For the previous 50 years of high and roughly-stable solar activity, no trends have been observed in CR data; however, as will be discussed later, the overall level of $\mathrm{CR}$ has changed significantly on the centurial-millennial timescales.

Full solution of the CR transport problems is a complicated task and requires sophisticated 3D time-dependent self-consistent modelling. However, the problem can be essentially simplified for applications at a long-timescale. An assumption on the azimuthal symmetry (requires times longer that the solar-rotation period) and quasisteady changes reduces it to a $2 \mathrm{D}$ quasi-steady problem. Further assumption of the spherical symmetry of the heliosphere reduces the problem to a 1D case. This approximation can be used only for rough estimates, since it neglects the drift effect, but it is useful for long-term studies, when the heliospheric parameters cannot be evaluated independently. Further, but still reasonable, assumptions (constant solar-wind speed, roughly power-law CR energy spectrum, slow spatial changes of the CR density) lead to the force-field approximation (Gleeson and Axford 1968; Caballero-Lopez and Moraal 2004), which can be solved analytically. The differential intensity $J_{i}$ of the cosmic-ray nuclei of type $i$ with kinetic energy $T$ at $1 \mathrm{AU}$ is given in this case as

$$
J_{i}(T, \phi)=J_{\mathrm{LIS}, i}\left(T+\Phi_{i}\right) \frac{(T)\left(T+2 T_{\mathrm{r}}\right)}{\left(T+\Phi_{i}\right)\left(T+\Phi_{i}+2 T_{\mathrm{r}}\right)},
$$

where $\Phi_{i}=\left(Z_{i} e / A_{i}\right) \phi$ for a cosmic nuclei of $i$-th type (charge and mass numbers are $Z_{i}$ and $A_{i}$ ), $T$ and $\phi$ are expressed in MeV/nucleon and in MV, respectively, $T_{\mathrm{r}}=938 \mathrm{MeV} . T$ is the CR particle's kinetic energy, and $\phi$ is the modulation potential. The local interstellar spectrum (LIS) $J_{\text {LIS }}$ forms the boundary condition for the heliospheric transport problem. Since LIS is not measured directly, i.e., outside the heliosphere, it is not well known in the energy range affected by CR modulation (below $100 \mathrm{GeV}$ ). Recent data from Voyager 1 and 2 spacecraft traveling beyond the termination shock give a clue for the lower-ebergy range of LIS (Webber et al. 2008; Bisschoff and Potgieter 2016), although the residual modulation beyond the heliopause may still affect this (Herbst et al. 2012). Presently-used approximations for LIS (e.g., Garcia-Munoz et al. 1975; Burger et al. 2000; Webber and Higbie 2003, 2009) agree with each other for energies above $20 \mathrm{GeV}$ but may contain uncertainties 
of up to a factor of 1.5 around $1 \mathrm{GeV}$. These uncertainties in the boundary conditions make the results of the modulation theory slightly model-dependent (see discussion in Usoskin et al. 2005; Herbst et al. 2010) and require the LIS model to be explicitly cited. This approach gives results, which are at least dimensionally consistent with the full theory and can be used for long-term studies ${ }^{1}$ (Usoskin et al. 2002a; Caballero-Lopez and Moraal 2004). Differential CR intensity is described by the only time-variable parameter, called the modulation potential $\phi$, which is mathematically interpreted as the averaged rigidity (i.e., the particle's momentum per unit of charge) loss of a CR particle in the heliosphere. However, it is only a formal spectral index whose physical interpretation is not straightforward, especially on short timescales and during active periods of the sun (Caballero-Lopez and Moraal 2004). Despite its cloudy physical meaning, this force-field approach provides a very useful and simple single-parametric approximation for the differential spectrum of GCR, since the spectrum of different GCR species directly measured near the Earth can be perfectly fitted by Eq. (3) using only the parameter $\phi$ in a wide range of solar activity levels (Usoskin et al. 2011). Therefore, changes in the whole energy spectrum (in the energy range from $100 \mathrm{MeV} /$ nucleon to $100 \mathrm{GeV} /$ nucleon) of cosmic rays due to the solar modulation can be described by this single number within the framework of the adopted LIS. The concept of modulation potential is a key concept for the method of solar-activity reconstruction by cosmogenic isotope proxy as it makes it possible to parameterize the GCR with one single parameter.

\subsubsection{Geomagnetic shielding}

Cosmic rays are charged particles and therefore are affected by the Earth's magnetic field. Thus the geomagnetic field puts an additional shielding on the incoming flux of cosmic rays. It is usually expressed in terms of the cutoff rigidity $P_{\mathrm{c}}$, which is the minimum rigidity a vertically incident CR particle must posses (on average) in order to reach the ground via secondaries of the cascade at a given location and time (Cooke et al. 1991). Neglecting such effects as the East-West asymmetry, which is roughly averaged out for the isotropic particle flux, or nondipole magnetic momenta, which decay rapidly with distance, one can come to a simple approximation, called the Störmer's equation, that describes the vertical geomagnetic cutoff rigidity $P_{\mathrm{c}}$ :

$$
P_{\mathrm{c}} \approx 1.9 M\left(R_{\mathrm{o}} / R\right)^{2} \cos ^{4} \lambda_{G}[\mathrm{GV}],
$$

where $M$ is the geomagnetic dipole moment (in $10^{25} \mathrm{G} \mathrm{cm}^{3}$ ), $R_{\mathrm{O}}$ is the Earth's mean radius, $R$ is the distance from the given location to the dipole center, and $\lambda_{G}$ is the geomagnetic latitude. The cutoff concept works like a Heaviside step-function so that all cosmic rays whose rigidity is below the cutoff are not allowed to enter the atmosphere while all particles with higher rigidity can penetrate. This approximation provides a good compromise between simplicity and reality (Nevalainen et al. 2013), especially when using the eccentric dipole description of the geomagnetic field (Fraser-

\footnotetext{
${ }^{1}$ Note that the famous work by Castagnoli and Lal (1980) contains an inconsistency in the force-field formula—see details in Usoskin et al. (2005).
} 
Smith 1987). The eccentric dipole has the same dipole moment and orientation as the centered dipole, but the dipole's center and consequently the poles, defined as crossings of the axis with the surface, are shifted with respect to geographical ones.

The shielding effect is the strongest at the geomagnetic equator, where the presentday value of $P_{\mathrm{c}}$ may reach up to $17 \mathrm{GV}$ in the region of India. There is almost no cutoff in the geomagnetic polar regions $\left(\lambda_{G} \geq 60^{\circ}\right)$. However, even in the latter case the atmospheric cutoff becomes important, i.e., particles must have rigidity above $0.5 \mathrm{GV}$ in order to initiate the atmospheric cascade which can reach ground (see Sect. 3.1.3).

The geomagnetic field is seemingly stable on the short-term scale, but it changes essentially on centurial-to-millennial timescales (e.g., Korte and Constable 2006). Such past changes can be evaluated based on measurements of the residual magnetization of independently-dated samples. These can be paleo- (i.e., natural stratified archives such as lake or marine sediments or volcanic lava) or archaeological (e.g., clay bricks that preserve magnetization upon baking) samples. Most paleo-magnetic data preserve not only the magnetic field intensity but also the direction of the local field, while archeo-magnetic samples provide information on the intensity only. Using a large database of such samples, it is possible to reconstruct (under reasonable assumptions) the large-scale magnetic field of the Earth. Data available provides good global coverage for the last three millennia, allowing for a reliable paleomagnetic reconstruction of the true dipole moment (DM) or virtual axial dipole moment ${ }^{2}$ (VADM) and its orientation (Licht et al. 2013). Less precise, but still reliable reconstructions of the DM and its orientation are possible for the last seven millennia (Knudsen et al. 2008; Usoskin et al. 2016a). Directional paleomagnetic reconstruction are less reliable on a longer timescale, because of the spatial sparseness of the paleo/archeo-magnetic samples in the earlier part of the Holocene (Korte et al. 2011). Some paleomagnetic reconstructions are shown in Fig. 5. All paleomagnetic models depict a similar longterm trend - an enhanced intensity during the period between $1500 \mathrm{BC}$ and $500 \mathrm{AD}$ and a significantly lower field before that.

Changes in the dipole moment $M$ inversely modulate the flux of CR at Earth, with strong effects in tropical regions and globally. The migration of the geomagnetic axis, which changes the geomagnetic latitude $\lambda_{G}$ of a given geographical location is also important; while not affecting the global flux of CR, it can dramatically change the CR effect regionally, especially at middle and high latitude. These changes affect the flux of CR impinging on the Earth's atmosphere both locally and globally and must be taken into account when reconstructing solar activity from terrestrial proxy data (Usoskin et al. 2008, 2010). Accounting for these effects is quite straightforward provided the geomagnetic changes in the past are known independently, e.g., from archeo and paleomagnetic studies (Donadini et al. 2010). However, because of progressively increasing uncertainties of paleomagnetic reconstructions back in time, it presently forms the main difficulty for the proxy method on the long-term scale (Snowball and Muscheler 2007), especially in the early part of the Holocene. On the other hand, the geomagnetic field variations are relatively well known for the last few millennia (Genevey et al. 2008; Korte and Constable 2008; Knudsen et al. 2008; Licht et al. 2013).

\footnotetext{
2 The concept of VADM assumes that the geomagnetic dipole is centered at the planet's center and its axis is aligned with the true magnetic axis.
} 
Fig. 5 Geomagnetic field intensity over millennia: VADM reconstructions over past 9000 years (a), with a zoom for the last 3200 years (b). Notations are: GMAG.9k (Usoskin et al. 2016a) with $1 \sigma$ (gray shading) and the full range variability (hatching); AF_M (Licht et al. 2013); G08 (Genevey et al. 2008); pfm9k.1b and pfm9k.1a (Nilsson et al. 2014); Kn08 (Knudsen et al. 2008); and SHA-DIF.14k (Pavón-Carrasco et al. 2014). Modified after (Usoskin et al. 2016a) (a)
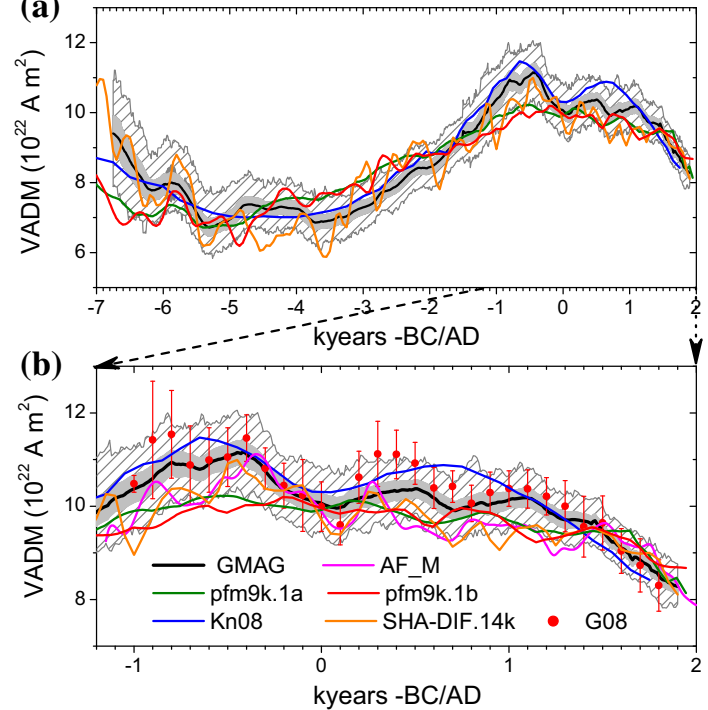

\subsubsection{Cosmic-ray-induced atmospheric cascade}

When an energetic CR particle enters the atmosphere, it first moves straight in the upper layers, suffering mostly from ionization energy losses that lead to the ionization of the ambient rarefied air and gradual deceleration of the particles. However, after traversing some amount of matter (the nuclear interaction mean-free path is on the order of $100 \mathrm{~g} / \mathrm{cm}^{2}$ for a proton in the air) the CR particle may collide with a nucleus in the atmosphere, producing a number of secondaries. These secondaries have their own fate in the atmosphere, in particular they may suffer further collisions and interactions forming an atmospheric cascade (e.g., Dorman 2004). Because of the thickness of the Earth's atmosphere $\left(1033 \mathrm{~g} / \mathrm{cm}^{2}\right.$ at sea level) the number of subsequent interactions can be large, leading to a fully-developed cascade (also called an air shower) consisting of secondary rather than primary particles. A schematic view of the atmospheric cascade is shown in Fig. 6. Three main components can be separated in the cascade:

- The "hadronic" nucleonic component is formed by the products of nuclear collisions of primary cosmic rays and their secondaries with the atmospheric nuclei, and consists mostly of superthermal protons and neutrons.

- The "soft" or electromagnetic component consists of electrons, positrons and photons.

- The "hard" or muon component consists mostly of muons; pions are short lived and decay almost immediately upon production, feeding muons and the "soft" component.

The development of the cascade depends mostly on the amount of matter traversed and is usually linked to residual atmospheric depth, which is very close to the static barometric pressure, rather than to the actual altitude, that may vary depending on the exact atmospheric density profile. 


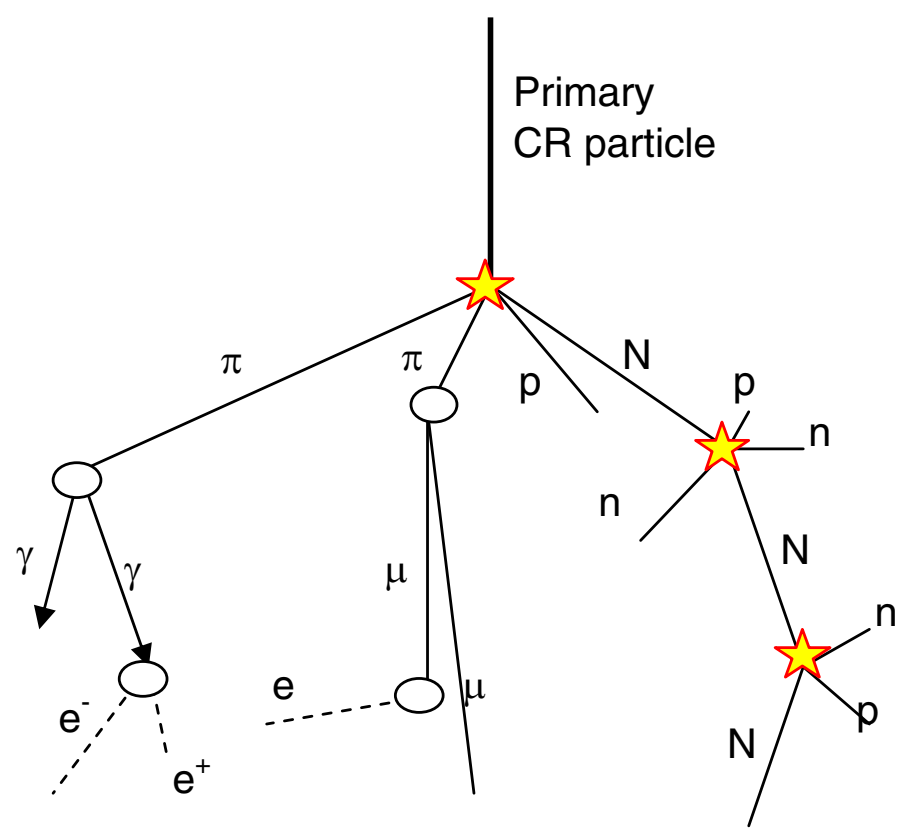

Fig. 6 Schematic view of an atmospheric cascade caused by energetic cosmic rays in the atmosphere. Leftto-right are denoted, respectively, the soft, muon and hadronic components of the cascade. Symbols "N, p, $\mathrm{n}, \mu, \pi, \mathrm{e}^{-}, \mathrm{e}^{+}$, and $\gamma$ " denote nuclei, protons, neutrons, muons, pions, electrons, positrons, and photons, respectively. Stars denote nuclear collisions, ovals-decay processes. This sketch does not represent the full development of the cascade and serves solely as an illustration for the processes discussed in the text. Image reproduced by permission from Usoskin (2011), copyright by SAIt

Cosmogenic isotopes are a by-product of the hadronic branch of the cascade (details are given below). Accordingly, in order to evaluate cosmic-ray flux from the cosmogenic isotope data, one needs to know the physics of cascade development. Several models have been developed for this cascade, in particular its hadronic branch with emphasis on the generation of cosmogenic isotope production. The first models were simplified quasi-analytical (e.g., Lingenfelter 1963; O'Brien and Burke 1973) or semiempirical models (e.g., Castagnoli and Lal 1980). With the fast advance of computing facilities it became possible to exploit the best numerical method suitable for such problems-Monte-Carlo (e.g., Masarik and Beer 1999, 2009; Webber and Higbie 2003; Webber et al. 2007; Usoskin and Kovaltsov 2008b; Kovaltsov and Usoskin 2010; Kovaltsov et al. 2012; Argento et al. 2013). The fact that models, based on different independent Monte-Carlo packages, namely, a general GEANT tool and a specific CORSIKA code, yield similar results provides additional verification of the approach.

\subsubsection{Transport and deposition}

A scheme for the transport and redistribution of the two most useful cosmogenic isotopes, ${ }^{14} \mathrm{C}$ and ${ }^{10} \mathrm{Be}$, is shown in Fig. 7. After a more-or-less similar production, the two isotopes follow quite different fates, as discussed in detail in Sects. 3.2.3 and 


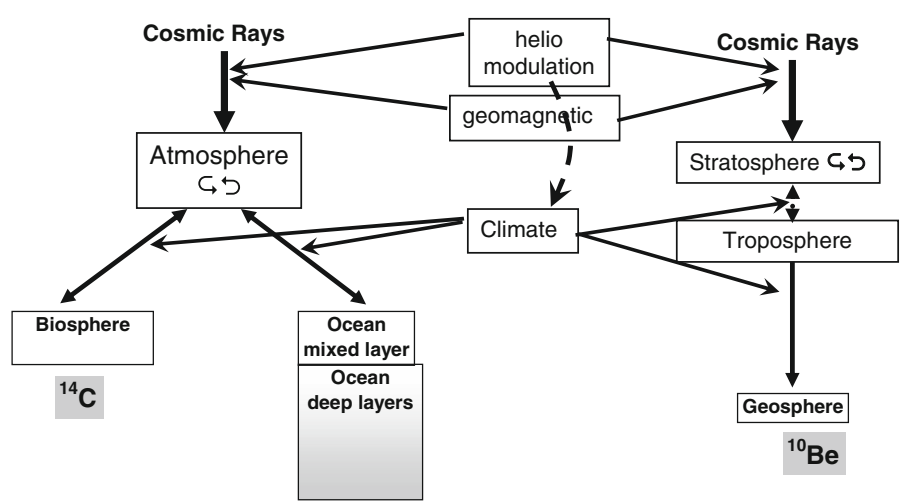

Fig. 7 Schematic representation of ${ }^{14} \mathrm{C}($ left $)$ and ${ }^{10} \mathrm{Be}(r i g h t)$ production chains. The flux of cosmic rays impinging on the Earth is affected by both heliospheric modulation and geomagnetic field changes. The climate may affect the redistribution of the isotopes between different reservoirs. Dashed line denotes a possible influence of solar activity on climate

3.3.3. Therefore, expected terrestrial effects are quite different for the isotopes and comparing them with each other can help in disentangling solar and climatic effects (see Sect. 3.7.3). A reader can find great detail also in a book by Beer et al. (2012).

\subsection{Radioisotope ${ }^{14} \mathrm{C}$}

The most commonly used cosmogenic isotope is radiocarbon ${ }^{14} \mathrm{C}$. This radionuclide is an unstable isotope of carbon with a half-life $\left(T_{1 / 2}\right)$ of about 5730 years. Since the radiocarbon method is extensively used in other science disciplines where accurate dating is a key issue (e.g., archeology, paleoclimatology, quaternary geology), it was developed primarily for this task. The solar-activity-reconstruction method, based on radiocarbon data, was initially developed as a by-product of the dating techniques used in archeology and Quaternary geology, in an effort to improve the quality of the dating by means of better information on the ${ }^{14} \mathrm{C}$ variable source function. The present-day radiocarbon calibration curve, based on a dendrochronological scale, uninterruptedly covers the whole Holocene (and extending to 50000 BP-Reimer et al. 2013) and provides a solid quantitative basis for studying solar activity variations on the multimillennial time scale.

\subsubsection{Measurements}

Radiocarbon is usually measured in tree rings, which allows an absolute dating of the samples by means of dendrochronology. Using a complicated technique, the ${ }^{14} \mathrm{C}$ activity $^{3} A$ is measured in an independently dated sample, which is then corrected for age as

\footnotetext{
${ }^{3}$ Isotope's activity quantifies (a) in the radiometric ${ }^{14} \mathrm{C}$ technique its decay rate, and is usually given in terms of disintegrations per minute per gram of carbon, and (b) in the AMS technique, the ${ }^{14} \mathrm{C} /{ }^{12} \mathrm{C}$ ratio, all normalized to the standard.
} 


$$
A^{*}=A \cdot \exp \left(\frac{0.693 t}{T_{1 / 2}}\right),
$$

where $t$ and $T_{1 / 2}$ are the age of the sample and the half-life of the isotope, respectively. Then the relative deviation from the standard activity $A_{o}$ of oxalic acid (the National Bureau of Standards) is calculated:

$$
\delta^{14} \mathrm{C}=\left(\frac{A^{*}-A_{o}}{A_{o}}\right) \cdot 1000 .
$$

After correction for the carbon isotope fractionating (account for the ${ }^{13} \mathrm{C}$ isotope) of the sample, the radiocarbon value of $\Delta^{14} \mathrm{C}$ is calculated (see details in Stuiver and Pollach 1977).

$$
\Delta^{14} \mathrm{C}=\delta^{14} \mathrm{C}-\left(2 \cdot \delta^{13} \mathrm{C}+50\right) \cdot\left(1+\delta^{14} \mathrm{C} / 1000\right),
$$

where $\delta^{13} \mathrm{C}$ is the per mille deviation of the ${ }^{13} \mathrm{C}$ content in the sample from that in the standard belemnite sample calculated similarly to Eq. (6). The value of $\Delta^{14} \mathrm{C}$ (measured in per mille $\%$ ) is further used as the index of radiocarbon relative activity. The series of $\Delta^{14} \mathrm{C}$ for the Holocene is presented in Fig. $8 \mathrm{a}$ as published by the IntCal13 collaboration of 21 dating laboratories as a result of systematic precise measurements of dated samples from around the world (Reimer et al. 2013) http://www.radiocarbon. org/IntCal13.htm. Panel b depicts the production rate $Q_{14 \mathrm{C}}$ reconstructed by Roth and Joos (2013) using the most up-to-date carbon cycle model.

A potentially interesting approach has been made by Lal et al. (2005), who measured the amount of ${ }^{14} \mathrm{C}$ directly produced by $\mathrm{CR}$ in polar ice. Although this method is free of the carbon-cycle influence, the first results, while being in general agreement with other methods, are not precise.

\subsubsection{Production}

The main source of radioisotope ${ }^{14} \mathrm{C}$ (except anthropogenic sources during the last decades) is cosmic rays in the atmosphere. It is produced as a result of the capture of a thermal neutron by atmospheric nitrogen

$$
{ }^{14} \mathrm{~N}+n \rightarrow{ }^{14} \mathrm{C}+p
$$

Neutrons are always present in the atmosphere as a product of the cosmic-ray-induced cascade (see Sect. 3.1.3) but their flux varies in time along with the modulation of cosmic-ray flux. This provides continuous source of the isotope in the atmosphere, while the sinks are isotope decay and transport into other reservoirs as described below (the carbon cycle).

The connection between the cosmogenic-isotope-production rate, $Q$, at a given location (quantified via the geomagnetic latitude $\lambda_{\mathrm{G}}$ ) and the cosmic-ray flux is given by

$$
Q=\int_{P_{\mathrm{c}}\left(\lambda_{\mathrm{G}}\right)}^{\infty} S(P, \phi) Y(P) d P,
$$




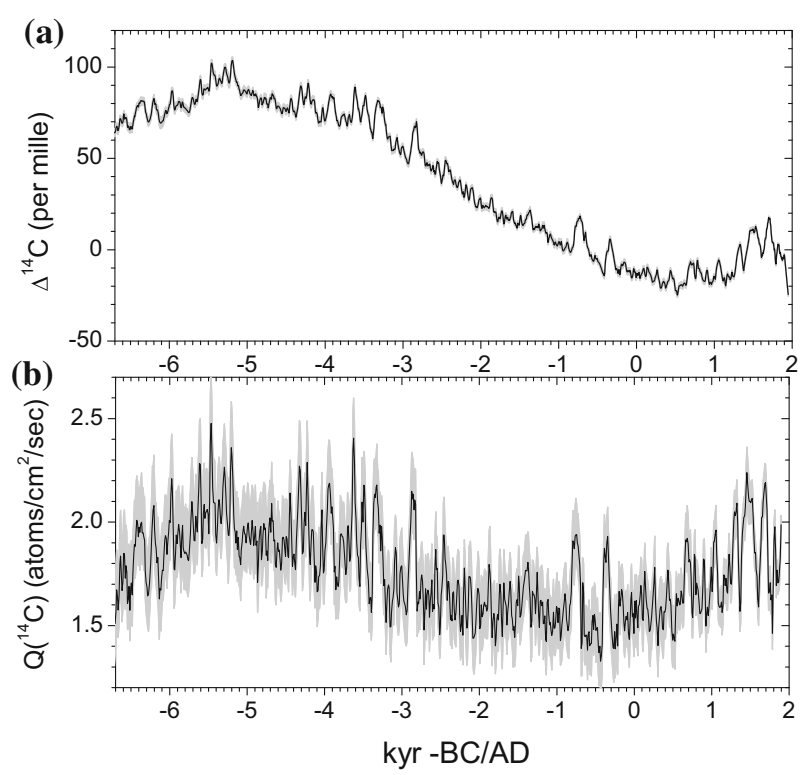

Fig. 8 Radiocarbon series for the Holocene. Upper panel Measured content of $\Delta^{14} \mathrm{C}$ in tree rings by IntCal13 collaboration (Reimer et al. 2013) (http://www.radiocarbon.org/IntCal13.htm). The long-term trend is caused by the geomagnetic field variations and the slow response of the oceans. Lower panel Production rate of ${ }^{14} \mathrm{C}$ in the atmosphere, reconstructed from the measured $\Delta{ }^{14} \mathrm{C}$, along with the $95 \%$ confidence interval (Roth and Joos 2013)

where $P_{\mathrm{c}}$ is the local cosmic-ray-rigidity cutoff (see Sect. 3.1.2), $S(P, \phi)$ is the differential energy spectrum of CR (see Sect. 3.1.1) and $Y(P)$ is the differential yield function of cosmogenic isotope production, calculated using a Monte-Carlo simulation of the cosmic-ray-induced atmospheric cascade (Kovaltsov et al. 2012; Poluianov et al. 2016). Because of the global nature of the carbon cycle and its long attenuation time, the radiocarbon is globally mixed before the final deposition, and Eq. (9) should be integrated over the globe. The yield function $Y(P)$ of the ${ }^{14} \mathrm{C}$ production is shown in Fig. 9a together with those for ${ }^{10} \mathrm{Be}$ (see Sect. 3.3.2) and for a ground-based neutron monitor (NM), which is the main instrument for studying cosmic-ray variability during the modern epoch. One can see that the yield function increases with the energy of CR. On the other hand, the energy spectrum of CR decreases with energy. Accordingly, the differential production rate (i.e., the product of the yield function and the spectrum, $F=Y \cdot S$ - the integrand of Eq. 9), shown in Fig. 9b, is more informative. The differential production rate reflects the sensitivity to cosmic rays, and the total production rate is simply an integral of $F$ over energy above the geomagnetic threshold.

Thanks to the development of atmospheric cascade models (Sect. 3.1.3), there are numerical models that allow one to compute the radiocarbon production rate as a function of the modulation potential $\phi$ and the geomagnetic dipole moment $M$. The overall production of ${ }^{14} \mathrm{C}$ is shown in Fig. 10.

The production rate of radiocarbon, $Q_{{ }^{14} \mathrm{C}}$, can vary as affected by different factors (see, e.g., Damon and Sonett 1991): 

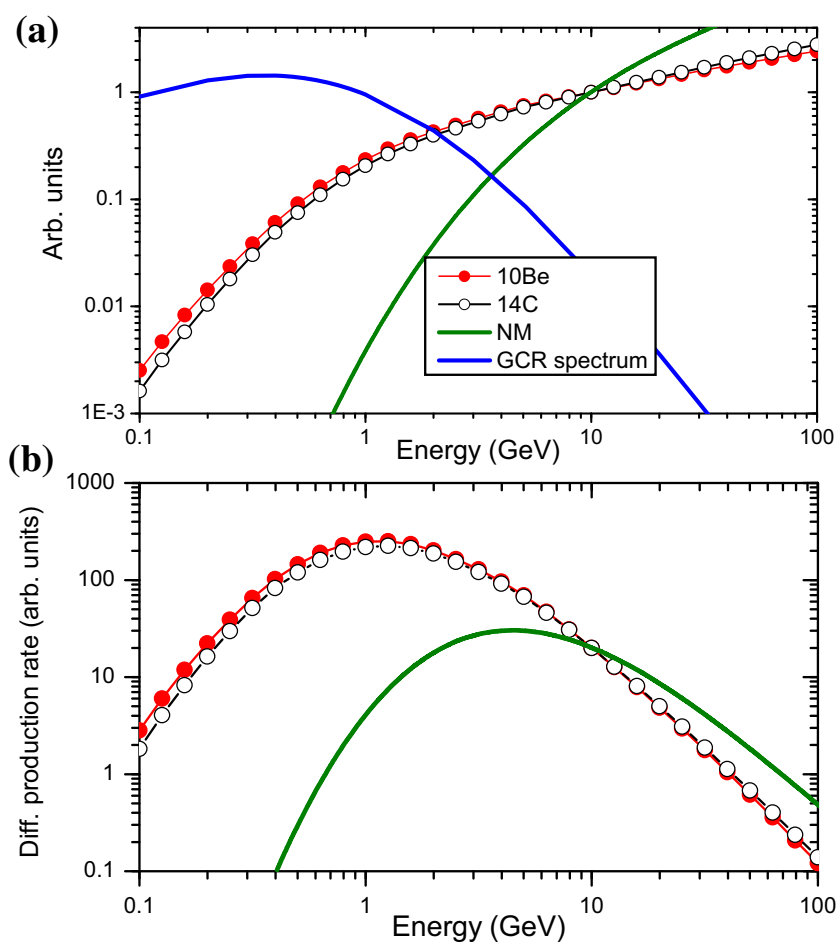

Fig. 9 Differential production yield functions of cosmogenic isotopes and ground-based neutron monitors as a function of cosmic-ray energy. a Columnar yield functions of ${ }^{10} \mathrm{Be}$ (Poluianov et al. 2016), ${ }^{14} \mathrm{C}$ (Poluianov et al. 2016), polar neutron monitor (Mishev et al. 2013) as well as the energy spectrum of galactic cosmic protons for medium modulation $(\phi=550 \mathrm{MV})$. b The differential production rate for ${ }^{10} \mathrm{Be}$ and ${ }^{14} \mathrm{C}$ cosmogenic isotopes, and the differential response function of a polar neutron monitor

- Variations of the cosmic-ray flux on a geological timescale due to the changing galactic background (e.g., a nearby supernova explosion or crossing the dense galactic arm).

- Secular-to-millennial variations are caused by the slowly-changing geomagnetic field. This is an important component of the variability, which needs to be independently evaluated from paleo and archeo-magnetic studies.

- Modulation of cosmic rays in the heliosphere by solar magnetic activity. This variation is the primary aim of the present method.

- Short-term variability of CR on a daily scale (suppression due to interplanetary transients or enhancement due to solar energetic-particle events) can be hardly resolved in radiocarbon data.

Therefore, the production rate of ${ }^{14} \mathrm{C}$ in the atmosphere can be modelled for a given time (namely, the modulation potential and geomagnetic dipole moment) and location. The global production rate $Q$ is then obtained as a result of global averaging.

Until recently there was a discrepancy between the modeled global-average ${ }^{14} \mathrm{C}$ production rates of 2.0-2.3 atoms $\mathrm{cm}^{-2} \mathrm{~s}^{-1}$ (see, e.g., O'Brien 1979; Masarik and Beer 1999; Goslar 2001; Usoskin et al. 2006b, and references therein) and the steady- 


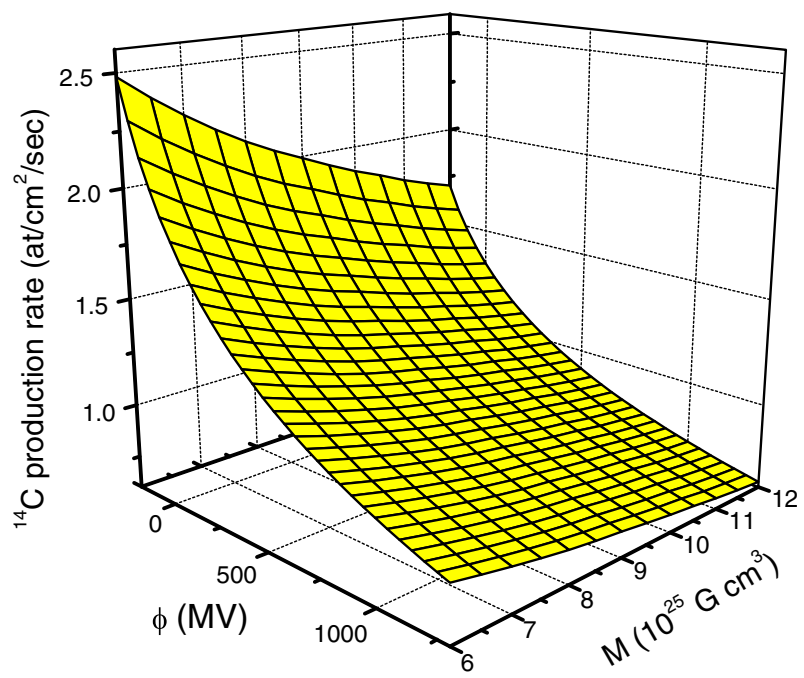

Fig. 10 Globally-averaged production rate of ${ }^{14} \mathrm{C}$ as a function of the modulation potential $\phi$ and geomagnetic dipole moment $M$, computed using the yield function by Kovaltsov et al. (2012), LIS by Burger et al. (2000) and cosmic-ray-modulation model by Usoskin et al. (2005). Other models (Masarik and Beer 2009; Poluianov et al. 2016) yield a similar result

state production calculated from the ${ }^{14} \mathrm{C}$ inventory in the carbon-cycle model (see Sect. 3.2.3) being 1.6-1.8 atoms $\mathrm{cm}^{-2} \mathrm{~s}^{-1}$ for the pre-industrial period (e.g.,Goslar 2001 , and references therein). The situation has been resolved recently with a new numerical model (Kovaltsov et al. 2012; Poluianov et al. 2016). For example, the mean global-averaged ${ }^{14} \mathrm{C}$ production rate for the period $1750-1900$ is estimated from measurements as $1.75 \pm 0.01$ atoms $\mathrm{cm}^{-2} \mathrm{~s}^{-1}$ (Roth and Joos 2013). The production model (Usoskin et al. 2016a; Poluianov et al. 2016) yields for the same period theoretical production rate $1.71-1.76$ atoms $\mathrm{cm}^{-2} \mathrm{~s}^{-1}$, depending on the solar activity and geomagnetic field reconstructions used, being thus in an excellent agreement with the data.

\subsubsection{Transport and deposition}

Upon production cosmogenic radiocarbon gets quickly oxidized to carbon dioxide $\mathrm{CO}_{2}$ and takes part in the regular carbon cycle of interrelated systems: atmospherebiosphere-ocean (Fig. 7). Because of the long residence time, radiocarbon becomes globally mixed in the atmosphere and involved in an exchange with the ocean. It is common to distinguish between an upper layer of the ocean, which can directly exchange $\mathrm{CO}_{2}$ with the air and deeper layers. The measured $\Delta^{14} \mathrm{C}$ comes from the biosphere (trees), which receives radiocarbon from the atmosphere. Therefore, the processes involved in the carbon cycle are quite complicated. The carbon cycle is usually described using a box model (Oeschger et al. 1974; Siegenthaler et al. 1980), where it is represented by fluxes between different carbon reservoirs and mixing within the ocean reservoir(s), as shown in Fig. 11. Production and radioactive decay are 


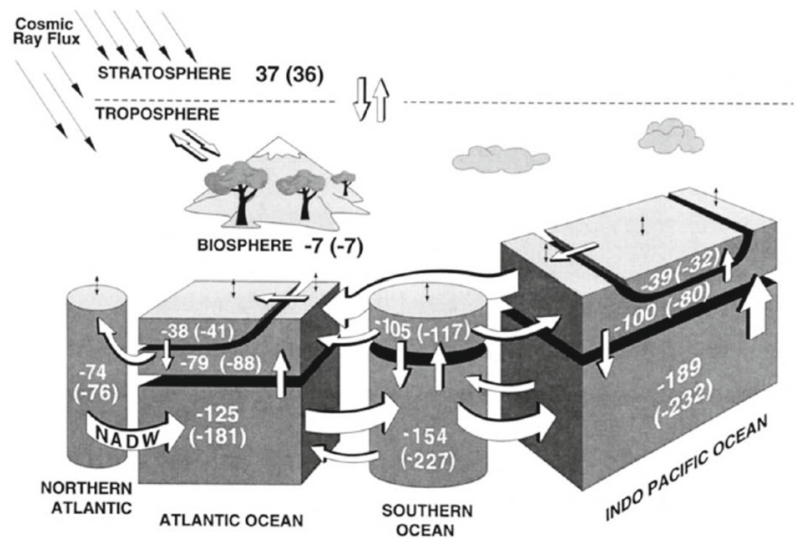

Fig. 11 A 12-box model of the carbon cycle (Broeker and Peng 1986; Siegenthaler et al. 1980). The number on each individual box is the steady-state $\Delta^{14} \mathrm{C}$ of this particular reservoir expressed in per mil. Image reproduced by permission from Bard et al. (1997), copyright by Elsevier

also included in box models. Free parameters in a typical box model are the ${ }^{14} \mathrm{C}$ production rate $Q$, the air-sea exchange rate (expressed as turnover rate $\kappa$ ), and the vertical-eddy-diffusion coefficient $K$, which quantifies ocean ventilation. Starting from the original representation (Oeschger et al. 1974), a variety of box models have been developed, which take into account subdivisions of the ocean reservoir and direct exchange between the deep ocean and the atmosphere at high latitudes. More complex models, including a diffusive approach, are able to simulate more realistic scenarios, but they require knowledge of a large number of model parameters. These parameters can be evaluated for the present time using the bomb test-studying the transport and distribution of the radiocarbon produced during the atmospheric nuclear tests. However, for long-term studies, only the production rate is considered variable, while the gas-exchange rate and ocean mixing are kept constant. Under such assumptions, there is no sense in subdividing reservoirs or processes, and a simple carbon box model is sufficient.

Using the carbon cycle model and assuming that all its parameters are constant in time, one can evaluate the production rate $Q$ from the measured $\Delta^{14} \mathrm{C}$ data. This assumption is well validated for the the Holocene (Damon et al. 1978; Stuiver et al. 1991) as there is no evidence of considerable oceanic change or other natural variability of the carbon cycle (Gerber et al. 2002), and accordingly all variations of $\Delta^{14} \mathrm{C}$ predominantly reflect the production rate. This is supported by the strong similarity of the fluctuations of the ${ }^{10} \mathrm{Be}$ data in polar ice cores (Sect. 3.3) compared to ${ }^{14} \mathrm{C}$, despite their completely different geochemical fate (Bard et al. 1997; Steinhilber et al. 2012). However, the changes in the carbon cycle during the last glaciation and deglaciation were dramatic, especially regarding ocean ventilation; this and the lack of independent information about the carbon cycle parameters, make it hardly possible to qualitatively estimate solar activity from ${ }^{14} \mathrm{C}$ before the Holocene.

A new-generation carbon cycle model has been developed moving from static box-exchange models to a dynamical model. Roth and Joos (2013) presented a fully featured model of intermediate complexity, named Bern3D-LPJ, which includes in 

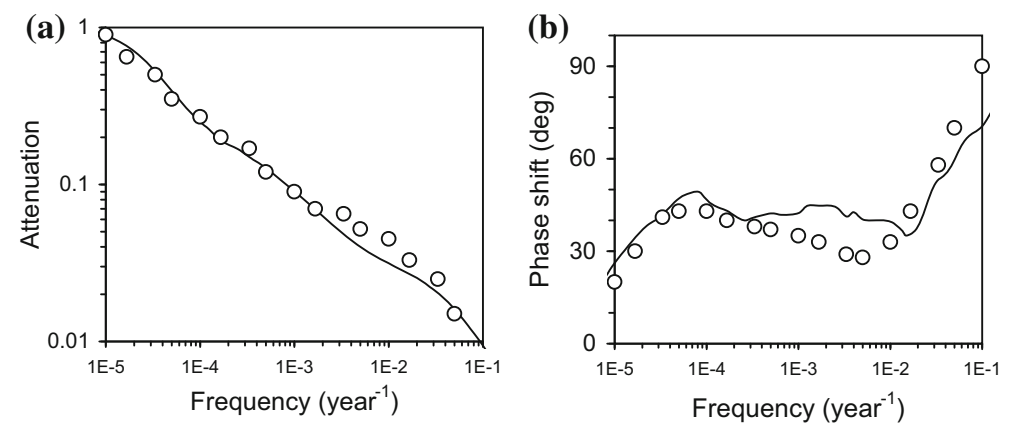

Fig. 12 The frequency characteristics of the carbon cycle: attenuation (left-hand panel) and phase shift (right-hand panel) as a function of the frequency of the ${ }^{14} \mathrm{C}$ production signal. Lines stand for a classical Oeschger-Siegenthaler box model (Siegenthaler et al. 1980), and open circles for a sophisticated PANDORA model (Bard et al. 1997)

addition to the dynamical atmosphere, a 3D dynamic ocean, ocean sediments, and a vegetation models. So far this is the most sophisticated and complete carbon cycle model. A multi-millennial reconstruction of the ${ }^{14} \mathrm{C}$ production rate, obtained as a result of application of this model to the IntCal09 radiocarbon data (Reimer et al. 2009) is shown in Fig. 8b.

First attempts to extract information on production-rate variations from measured $\Delta^{14} \mathrm{C}$ were based on simple frequency separations of the signals. All slow changes were ascribed to climatic and geomagnetic variations, while short-term fluctuations were believed to be of solar origin. This was done by removing the long-term trend from the $\Delta^{14} \mathrm{C}$ series and claiming the residual as being a series of solar variability (e.g., Peristykh and Damon 2003). This oversimplified approach was natural at earlier times, before the development of carbon cycle models, but later it was replaced by the inversion of the carbon cycle (i.e., the reconstruction of the production rate from the measured ${ }^{14} \mathrm{C}$ concentration). Although mathematically this problem can be solved correctly as a system of linear differential equations, the presence of fluctuating noise with large magnitude makes it not straightforward, since the time derivative cannot be reliably identified leading thus to possible amplification of the high-frequency noise in $\Delta^{14} \mathrm{C}$ data. One traditional approach (e.g., Stuiver and Quay 1980) is based on an iterative procedure, first assuming a constant production rate, and then fitting the calculated $\Delta^{14} \mathrm{C}$ variations to the actual measurements using a feedback scheme. A concurrent approach based on the presentation of the carbon cycle as a Fourier filter (Usoskin and Kromer 2005) produces similar results. Roughly speaking, the carbon cycle acts as an attenuating and delaying filter for the ${ }^{14} \mathrm{C}$ signal (see Fig. 12). The higher the frequency is, the greater the signal is attenuated. In particular, the large 11year solar cycle expected in the ${ }^{14} \mathrm{C}$ is attenuated by a factor of hundred in the measured $\Delta{ }^{14} \mathrm{C}$ data, making it hardly detectable. Because of the slow oceanic response, the ${ }^{14} \mathrm{C}$ data is also delayed with respect to the production signal. The production rate $Q^{14} \mathrm{C}$ for the Holocene is shown in Fig. 8 and depicts both short-term fluctuations as well as slower variations, mostly due to geomagnetic field changes (see Sect. 3.2.5). 


\subsubsection{The Suess effect and nuclear bomb tests}

Unfortunately, cosmogenic ${ }^{14} \mathrm{C}$ data cannot be easily used for the last century, primarily because of the extensive burning of fossil fuels. Since fossil fuels do not contain ${ }^{14} \mathrm{C}$, the produced $\mathrm{CO}_{2}$ dilutes the atmospheric ${ }^{14} \mathrm{CO}_{2}$ concentration with respect to the preindustrial epoch. Therefore, the measured $\Delta^{14} \mathrm{C}$ cannot be straightforwardly translated into the production rate $Q$ after the late nineteenth century, and a special correction for fossil fuel burning is needed. This effect, known as the Suess effect (e.g., Suess 1955), can be up to $-25^{\circ} \%$ in $\Delta^{14} \mathrm{C}$ in 1950 (Tans et al. 1979), which is an order of magnitude larger than the amplitude of the 11-year cycle of a few $\%$. Moreover, while the cosmogenic production of ${ }^{14} \mathrm{C}$ is roughly homogeneous over the globe and time, the use of fossil fuels is highly nonuniform (e.g., de Jong and Mook 1982) both spatially (developed countries, in the northern hemisphere) and temporarily (World Wars, Great Depression, industrialization, etc.). This makes it very difficult to perform an absolute normalization of the radiocarbon production to the direct measurements. Sophisticated numerical models (e.g., Sabine et al. 2004; Mikaloff Fletcher et al. 2006) aim to account for the Suess effect and make good progress. However, the results obtained indicate that the determination of the Suess effect does not yet reach the accuracy required for the precise modelling and reconstruction of the ${ }^{14} \mathrm{C}$ production for the industrial epoch. Note that the atmospheric concentration of another carbon isotope ${ }^{13} \mathrm{C}$ is partly affected by land use, which has also been modified during the last century.

Another anthropogenic activity greatly disturbing the natural variability of ${ }^{14} \mathrm{C}$ is related to the atmospheric nuclear bomb tests actively performed in the 1960s. For example, the radiocarbon concentration nearly doubled in the early 1960s in the northern hemisphere after nuclear tests performed by the USSR and the USA in 1961 (Damon et al. 1978). On one hand, such sources of momentary spot injections of radioactive tracers (including ${ }^{14} \mathrm{C}$ ) provide a good opportunity to verify and calibrate the exchange parameters for different carbon-cycle reservoirs and circulation models (e.g., Bard et al. 1987; Sweeney et al. 2007). Thus, the present-day carbon cycle is more-or-less known. On the other hand, the extensive additional production of isotopes during nuclear tests makes it hardly possible to use the ${ }^{14} \mathrm{C}$ as a proxy for solar activity after the 1950s (Joos 1994).

These anthropogenic effects do not allow one to make a straightforward link between pre-industrial data and direct experiments performed during more recent decades. Therefore, the question of the absolute normalization of ${ }^{14} \mathrm{C}$ model is still open (see, e.g., the discussion in Solanki et al. 2004, 2005; Muscheler et al. 2005).

\subsubsection{The effect of the geomagnetic field}

As discussed in Sect. 3.1.2, knowledge of geomagnetic shielding is an important aspect of the cosmogenic isotope method. Since radiocarbon is globally mixed in the atmosphere before deposition, its production is affected by changes in the geomagnetic dipole moment $M$, while magnetic-axis migration plays hardly any role in ${ }^{14} \mathrm{C}$ data.

The crucial role of paleomagnetic reconstructions has long been known (e.g., Elsasser et al. 1956; Kigoshi and Hasegawa 1966). Many earlier corrections for pos- 
sible geomagnetic-field changes were performed by detrending the measured $\Delta^{14} \mathrm{C}$ abundance or production rate $Q$ (Stuiver and Quay 1980; Voss et al. 1996; Peristykh and Damon 2003), under the assumption that geomagnetic and solar signals can be disentangled from the production in the frequency domain. Accordingly, the temporal series of either measured $\Delta^{14} \mathrm{C}$ or its production rate $Q$ is decomposed into the slow changing trend and faster oscillations. The trend is supposed to be entirely due to geomagnetic changes, while the oscillations are ascribed to solar variability. Such a method, however, obliterates all information on possible long-term variations of solar activity. On the other hand, this also misinterprets the short-term (centennial timescale) variations of the geomagnetic field which are essential (e.g., Licht et al. 2013). Accordingly, the frequency-domain decomposition may lead to erroneous results. A direct correction for the geomagnetic field effect should be used instead.

Simplified empirical correction factors were also often used (e.g., Stuiver and Quay 1980; Stuiver et al. 1991). The modern approach is based on a physics-based model (e.g., Solanki et al. 2004; Vonmoos et al. 2006) and allows the quantitative reconstruction of solar activity, explicitly using independent reconstructions of the geomagnetic field. In this case the major source of errors in solar activity reconstructions is related to uncertainties in the paleomagnetic data (Snowball and Muscheler 2007). These errors are insignificant for the last several millennia (Licht et al. 2013; Usoskin et al. 2016a), but become increasingly important for earlier times.

\subsection{Cosmogenic isotope ${ }^{10} \mathrm{Be}$}

\subsubsection{Measurements}

The cosmogenic isotope ${ }^{10} \mathrm{Be}$ is useful for long-term studies of solar activity because of its long half-life of around $1.5 \times 10^{6}$ years. Its concentration is usually measured in stratified ice cores allowing for independent dating. The ${ }^{10} \mathrm{Be} /{ }^{9} \mathrm{Be}$ ratio needs to be precisely measured at an accuracy better than $10^{-13}$. This can be done using AMS (Accelerator Mass Spectrometry) technique, which makes the measurements complicated and expensive. Correction for the decay is straightforward and does not include isotope fractionating. From the measured samples, first the ${ }^{10} \mathrm{Be}$ concentration is defined, usually in units of $10^{4}$ atoms/g. Sometimes, a correction for the snow precipitation amount is considered leading to the observable ${ }^{10} \mathrm{Be}$ flux, which is the number of atoms, precipitating to the surface per $\mathrm{cm}^{2}$ per second.

There exist different ${ }^{10} \mathrm{Be}$ series suitable for studies of long-term solar activity, coming from ice cores in Greenland and Antarctica. They have been obtained from different cores with different resolutions, and include data from Milcent, Greenland (Beer et al. 1983); Camp Century, Greenland (Beer et al. 1988); Dye 3, Greenland (Beer et al. 1990); Dome Concordia and South Pole, Antarctica (Raisbeck et al. 1990); GRIP, Greenland (Yiou et al. 1997); GISP2, Greenland (Finkel and Nishiizumi 1997); Dome Fuji, Antarctica (Horiuchi et al. 2007, 2008; Miyake et al. 2015); Dronning Maud Land, Antarctica (Ruth et al. 2007); NGRIP (North Greenland Ice Core Project), Greenland (Berggren et al. 2009); NEEM (North Greenland Eemian Ice Drilling), 
Greenland (Sigl et al. 2015); West Antarctic Ice Sheet Divide Ice Core (WAIS/WDC), Antarctica (Sigl et al. 2015), etc.

We note that data on ${ }^{10} \mathrm{Be}$ in other archives, e.g., lake sediments, is usually more complicated to interpret because of the potential influence of the climate (Horiuchi et al. 1999; Belmaker et al. 2008).

Details of the ${ }^{10} \mathrm{Be}$ series and their comparison with each other can be found in Beer (2000), Muscheler et al. (2007), and Beer et al. (2012).

\subsubsection{Production}

The isotope ${ }^{10} \mathrm{Be}$ is produced as a result of spallation of atmospheric nitrogen and oxygen (carbon is less abundant in the atmosphere and makes a negligible contribution) by the nucleonic component of the cosmic-ray-induced atmospheric cascade (Sect. 3.1.3).

A small contribution may also exist from photo-nuclear reactions (Bezuglov et al. 2012). The cross section (a few mb) of the spallation reactions is almost independent of the energy of impacting particles and has a threshold of about $15 \mathrm{MeV}$. Thus, the production of ${ }^{10} \mathrm{Be}$ is defined mostly by the multiplicity of the nucleonic component, which increases with the energy of primary cosmic rays (see Fig. 9). Maximum production occurs at an altitude of $10-15 \mathrm{~km}$ due to a balance between the total energy of the cascade (which increases with altitude) and the number of secondaries (decreasing with altitude). Most of the global ${ }^{10} \mathrm{Be}$ is produced in the stratosphere $(55-70 \%)$ and the rest in the troposphere (Lal and Peters 1967; Masarik and Beer 1999, 2009; Usoskin and Kovaltsov 2008b; Kovaltsov and Usoskin 2010).

Computation of ${ }^{10} \mathrm{Be}$ isotope production is straightforward, provided a model of the atmospheric cascade is available. The first consistent model was developed by D. Lal et al. (Bhandari et al. 1966; Lal and Peters 1967; Lal and Suess 1968), using an empirical approach based on fitting simplified model calculations to measurements of the isotope concentrations and "star" (inelastic nuclear collisions) formations in the atmosphere. Next was an analytical model by O'Brien (1979), who solved the problem of the GCR-induced cascade in the atmosphere using an analytical stationary approximation in the form of the Boltzmann equation. Those models were based on calculating the rate of inelastic collisions or "stars" and then applying the mean spallation yield per "star". A new step in the modelling of isotope production was made by Masarik and Beer (1999), who performed a full Monte-Carlo simulation of a GCR-initiated cascade in the atmosphere and used cross sections of spallation reactions directly instead of the average "star" efficiency. Modern models (Webber and Higbie 2003; Webber et al. 2007; Usoskin and Kovaltsov 2008b; Kovaltsov and Usoskin 2010; Poluianov et al. 2016) are based on a full Monte-Carlo simulation of the atmospheric cascade, using improved cross sections. The global production rate of ${ }^{10} \mathrm{Be}$ is about $0.02-0.04$ atoms cm${ }^{-2} \mathrm{~s}^{-1}$ (Masarik and Beer 1999; Webber et al. 2007; Kovaltsov and Usoskin 2010; Poluianov et al. 2016), which is lower than that for ${ }^{14} \mathrm{C}$ (about 2 atoms $\mathrm{cm}^{-2} \mathrm{~s}^{-1}$; see Sect. 3.2.2) by two orders of magnitude. The yield function of ${ }^{10} \mathrm{Be}$ production is shown in Fig. 9a and the differential production rate in Fig. 9b. One can see that the peak of ${ }^{10} \mathrm{Be}$ sensitivity, especially in polar regions, is shifted towards lower energies (below $1 \mathrm{GeV}$ ) compared with a neutron monitor. 
This implies that the ${ }^{10} \mathrm{Be}$ isotope is relatively more sensitive to less energetic $\mathrm{CR}$ and is, therefore, more affected by solar energetic particles (Usoskin et al. 2006b). Comparison of model computations with direct beryllium production experiments (Usoskin and Kovaltsov 2008b; Kovaltsov and Usoskin 2010), and also the results of modelling of the short-living ${ }^{7} \mathrm{Be}$ isotope (Usoskin et al. 2009a) suggest that some numerical models (Masarik and Beer 1999; Webber and Higbie 2003; Webber et al. 2007) tend to underestimate the production.

Although the production of ${ }^{10} \mathrm{Be}$ can be more-or-less precisely modelled, a simple normalization "surface", similar to that shown in Fig. 10 for ${ }^{14} \mathrm{C}$, is not easy to produce because of partial mixing in the atmosphere (see Sect. 3.3.3). Simplified models, assuming either only global (e.g., Beer 2000) or polar production (Bard et al. 1997; Usoskin et al. 2004), have been used until recently. However, it has been recognized that a more realistic model of the limited atmospheric mixing should be used. Without detailed knowledge of ${ }^{10} \mathrm{Be}$ transport in the atmosphere, it is impossible to relate the quantitatively-measured concentration to the production (as done for ${ }^{14} \mathrm{C}$ using the carbon cycle), and one has to assume that the measured abundance is proportional (with an unknown coefficient) to the production rate in a specific geographical region (see Sect. 3.3.3).

\subsubsection{Atmospheric transport}

After production, the ${ }^{10} \mathrm{Be}$ isotope has a seemingly simple (Fig. 7) but difficultto-account-for fate in the atmosphere. Its atmospheric residence time depends on scavenging, stratosphere-troposphere exchange and inter-tropospheric mixing (e.g., McHargue and Damon 1991). Soon after production, the isotope is thought to become attached to atmospheric aerosols and follows their fate (Beer et al. 2012). In addition, it may be removed from the lower troposphere by wet deposition (rain and snow). The mean residence time of the aerosol-bound radionuclide in the atmosphere is quite different for the troposphere, being a few weeks, and stratosphere, where it is one to two years (Raisbeck et al. 1981). Accordingly, ${ }^{10} \mathrm{Be}$ produced in the troposphere is deposited mostly locally, i.e., in the polar regions, while stratospheric ${ }^{10} \mathrm{Be}$ can be partly or totally mixed. In addition, because of the seasonal (usually Spring) intrusion of stratospheric air into the troposphere at mid-latitudes, there is an additional contribution of stratospheric ${ }^{10} \mathrm{Be}$. Therefore, the measured ${ }^{10} \mathrm{Be}$ concentration (or flux) in polar ice is modulated not only by production but also by climate/precipitation effects (e.g., Steig et al. 1996; Bard et al. 1997). This led Lal (1987) to the extreme conclusion that variations of polar ${ }^{10} \mathrm{Be}$ reflect a meteorological, rather than solar, signal. However, comparison between Greenland and Antarctic ${ }^{10} \mathrm{Be}$ series and between ${ }^{10} \mathrm{Be}$ and ${ }^{14} \mathrm{C}$ data (e.g., Bard et al. 1997; Horiuchi et al. 2008; Beer et al. 2012; Steinhilber et al. 2012) suggests that the beryllium data mostly depicts production variations (i.e., solar signal) on top of which some meteorological effects can be superposed (see also Sect. 3.7.3).

Since both assumptions of the global and purely-local polar production of ${ }^{10} \mathrm{Be}$ archived in polar ice are over-simplified, several attempts have been made to overcome this problem. For instance, McCracken (2004) proposed several simple mathematical models of partial atmospheric mixing (without division in the troposphere and strato- 
sphere) and compared them with observed data. From this semi-empirical approach McCracken concluded that M2 (full mixing above $60^{\circ}$ latitude and a limited mixing between $40^{\circ}$ and $60^{\circ}$ latitude) is a reasonable model for Antarctica. Vonmoos et al. (2006) assumed that the production of ${ }^{10} \mathrm{Be}$ recorded in Greenland is related to the entire hemisphere in the stratosphere (i.e, global stratospheric mixing) but is limited to latitudes above $40^{\circ}$ latitude in the troposphere (partial tropospheric mixing). This approach uses either semi-empirical or indirect arguments in choosing the unknown degree of mixing.

Recent efforts in employing modern atmospheric 3D circulation models for simulations of ${ }^{10} \mathrm{Be}$ transport and deposition, including realistic air-mass transport and dry-vs-wet deposition (Field et al. 2006; Heikkilä et al. 2008, 2009), look more promising. An example of ${ }^{10} \mathrm{Be}$ deposition computed on the world grid using the NASA GISS model (Field et al. 2006) is shown in Fig. 13. Precision of the models allows one to distinguish local effects, e.g., for Greenland (Heikkilä et al. 2008). A simulation performed by combining a detailed ${ }^{10} \mathrm{Be}$-production model with an air-dynamics model can result in an absolute model relating production and deposition of the radionuclide. The validity and usefulness of this approach has been demonstrated by Usoskin et al. (2009a), who directly modeled production (using the CRAC model-Usoskin and Kovaltsov 2008b) and transport (using the GISS ModelE-Koch et al. 2006) of a short-living beryllium isotope ${ }^{7} \mathrm{Be}$ and showed that such a combined model is able to

Fig. 13 Wet (a) and dry (b) deposition of ${ }^{10} \mathrm{Be}$, computed using the NASA GISS model (Field et al. 2006) for a fixed sea-surface temperature (a) Annual mean wet ${ }^{10}$ Be deposition $\left(10^{-27} \mathrm{~kg} / \mathrm{m}^{2} / \mathrm{s}\right)$

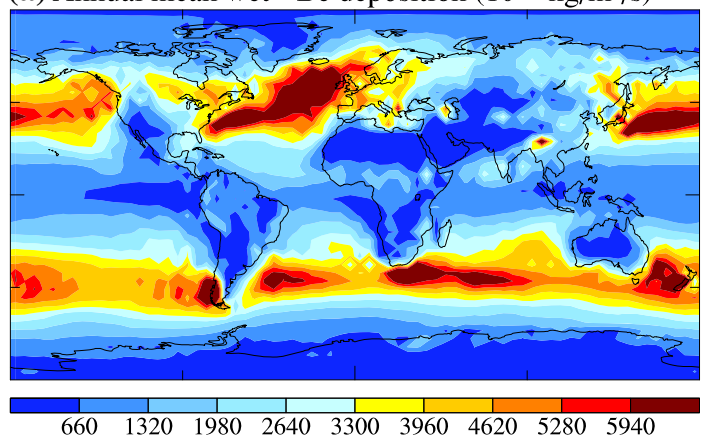

(b) Annual mean dry ${ }^{10} \mathrm{Be}$ deposition $\left(10^{-27} \mathrm{~kg} / \mathrm{m}^{2} / \mathrm{s}\right)$

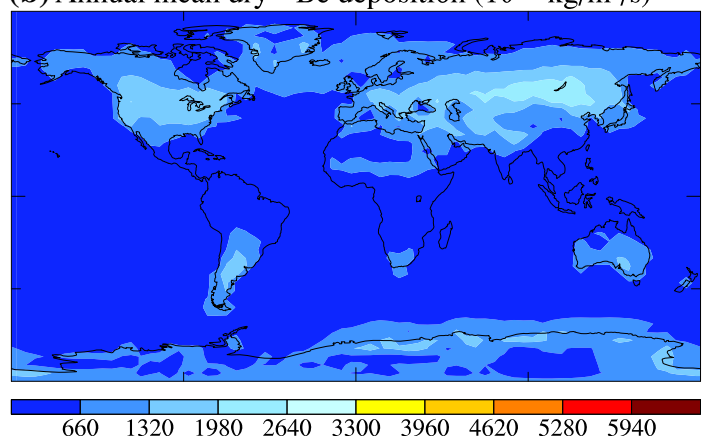


correctly reproduce both the absolute level and temporal variations of the ${ }^{7} \mathrm{Be}$ concentration measured in near ground air around the globe. Keeping in mind the similarity between production and transport of the two beryllium isotopes, ${ }^{7} \mathrm{Be}$ and ${ }^{10} \mathrm{Be}$, this serves as support for the advanced modelling of ${ }^{10} \mathrm{Be}$ transport. A similar general agreement between measured and modelled seasonal variability has been recently found for ${ }^{10} \mathrm{Be}$ in an Antarctic ice core (Pedro et al. 2011).

\subsubsection{Effect of the geomagnetic field}

In order to properly account for geomagnetic changes (Sect. 3.1.2), one needs to know the effective region in which the radionuclide is produced before being stored in the archive analyzed. For instance, if the concentration of ${ }^{10} \mathrm{Be}$ measured in polar ice reflects mainly the isotope's production in the polar atmosphere (as, e.g., assumed by Usoskin et al. 2003c), no strong geomagnetic signal is expected to be observed, since the geographical poles are mostly related to high geomagnetic latitudes. On the other hand, assuming global mixing of atmospheric ${ }^{10} \mathrm{Be}$ before deposition in polar ice (e.g., Masarik and Beer 1999), one expects that only changes in the geomagnetic dipole moment affect will the signal. However, because of partial mixing, which can be different in the stratosphere and troposphere, taking into account migration and displacement of the geomagnetic dipole axis may be essential for a reliable reconstruction of solar variability from ${ }^{10} \mathrm{Be}$ data (McCracken 2004). Therefore, only a full combination of the transport and production models, the latter explicitly including geomagnetic effects estimated from paleomagnetic reconstructions, can adequately account for geomagnetic changes and separate the solar signal. These forms a new generation of physics-based models for the cosmogenic-isotope proxy method. We note that paleomagnetic data should ideally not only provide the dipole moment (VADM or VDM) but should also provide estimates of the geomagnetic axis attitude and displacement of the dipole center (Korte et al. 2011).

\subsection{Other potential proxy}

An interesting new potential proxy for solar activity (or cosmic ray) variability on the long-term centennial-to-millennial time scale has been proposed recently by Traversi et al. (2012). This is the nitrate content in a polar ice core Talos Dome in Antarctica, which has a favorite location in the sense of snow accumulation and conservation of such volatile specie as nitrate. Nitrate-related species are partly produced in the stratosphere/troposphere as a result of the ionization of the atmospheric air by cosmic rays and, partly, via terrestrial sources (e.g., lightnings) and are subject to air transport (Rozanov et al. 2012). As shown by Traversi et al. (2012), the nitrate concentration/flux measured in the Talos Dome ice core for the Holocene period agrees well with the cosmogenic data of ${ }^{14} \mathrm{C}$ in tree rings and ${ }^{10} \mathrm{Be}$ in both Antarctic and Greenland ice cores, on the time scales from centennia to millennia. Due to the large errors of the ice core dating, 200-300 years (Schüpbach et al. 2011), shorter time scales cannot be considered. The level of the nitrate variability is generally consistent with that predicted by theoretical models assuming its production by GCR in the atmosphere 
(Semeniuk et al. 2011; Rozanov et al. 2012). The ability of nitrate to catch up longterm cosmic ray variability has been confirmed also by an analysis of nitrate and ${ }^{10} \mathrm{Be}$ data for the Laschamp event ca. 40 kyears ago (Traversi et al. 2016). Thus, the nitrate in an ice core provides a potential to become a new proxy of long-term solar activity, with independent atmospheric fate, which would strengthen the robustness of the reconstructions. However, an independent confirmation of the result and a more detailed model are needed before it can serve as a new quantitative proxy. Note that the mechanism of the nitrate production and transport is not related to the possible nitrate peaks claimed to be caused by strong solar energetic-particle events (see Sect. 5.3).

\subsection{Towards a quantitative physical model}

Several methods have been developed historically to convert measured cosmogenicisotope data into a solar activity index, ranging from very simple regressions to physics-based models. A new step in long-term solar-activity reconstruction has been made recently, which is the development of the proxy method in which physicsbased models are used, instead of a phenomenological regression, to link SN with cosmogenic-isotope production (Usoskin et al. 2003c, 2007, 2014, 2016a; Solanki et al. 2004; Vonmoos et al. 2006; Muscheler et al. 2007; Steinhilber et al. 2012). Due to recent theoretical developments, it is now possible to construct a chain of physical models to model the entire relationship between solar activity and cosmogenic data. A multi-proxy approach based on different cosmogenic proxy data combined in a joint reconstruction is progressive (Steinhilber et al. 2012).

The physics-based reconstruction of solar activity (in terms of sunspot numbers) from cosmogenic proxy data includes several steps:

- Computation of the isotope's production rate in the atmosphere from the measured concentration in the archive (Sects. 3.2.2 and 3.3.2);

- Computation, considering independently-known secular geomagnetic changes (see Sect. 3.2.5) and a model of the CR-induced atmospheric cascade, of the GCR spectrum parameter quantified via the modulation potential $\phi$ (Sect. 3.5.2), some reconstructions being terminated at this point;

- Computation of a heliospheric index, whether of the open solar magnetic flux or of the average HMF intensity at the Earth's orbit (Sect. 3.5.2)

- Computation of a solar index (sunspot number series), corresponding to the abovederived heliospheric parameter (Sect. 3.5.3).

Presently, all these steps can be completed using appropriate models. Some models stop after computations of the modulation potential as its translation into the solar index may include additional uncertainties. Although the uncertainties of the models may be considerable, the models allow a full basic quantitative reconstruction of solar activity in the past. However, much needs to be done, both theoretically and experimentally, to obtain an improved reconstruction. 
(a)

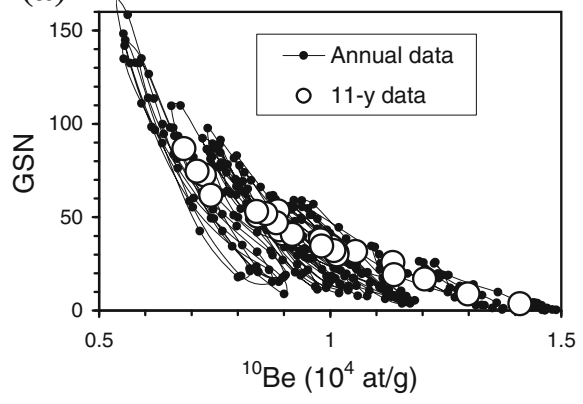

(b)

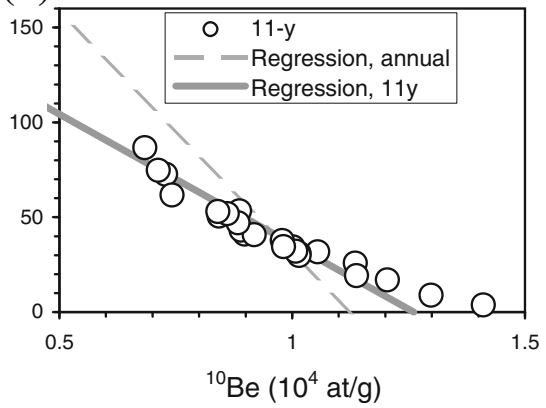

Fig. 14 Scatter plot of smoothed group sunspot numbers versus (2-year delayed) ${ }^{10} \mathrm{Be}$ concentration. a Annual (connected small dots) and 11-year averaged (big open dots) values. b Best-fit linear regressions between the annual (dashed line) and 11-year averaged values (solid line). The dots are the same as in panel (a). (After Usoskin and Kovaltsov 2004)

\subsubsection{Regression models}

Mathematical regression is the most apparent and often used (even recently) method of solar-activity reconstruction from proxy data (see, e.g., Stuiver and Quay 1980; Ogurtsov 2004). The reconstruction of solar activity is performed in two consecutive steps. First, a phenomenological regression (either linear or nonlinear) is built between a proxy data set and a direct solar-activity index for the available "training" period (e.g., since 1750 for WSN or since 1610 for GSN). Then this regression is extrapolated backwards to evaluate $\mathrm{SN}$ from the proxy data. The main shortcoming of the regression method is that it depends on the time resolution and choice of the "training" period. The former is illustrated by Fig. 14, which shows the scatter plot of the ${ }^{10} \mathrm{Be}$ concentration versus GSN for the annual and 11-year smoothed data. One can see that the slope of the ${ }^{10} \mathrm{Be}-\mathrm{vs}-\mathrm{GSN}$ relation (about $-500 \mathrm{~g} / \mathrm{atom}$ ) within individual cycles is significantly different from the slope of the long-term relation (about $-100 \mathrm{~g} / \mathrm{atom}$ ), i.e., individual cycles do not lie on the line of the 11-year averaged cycles. Moreover, the slope of the regression for individual 11-year cycles varies essentially depending on the solar activity level. Therefore, a formal regression built using the annual data for 1610-1985 yields a much stronger GSN-vs- ${ }^{10}$ Be dependence than for the cycle-averaged data (see Fig. 14b), leading to a potentially-erroneous evaluation of the sunspot number from the ${ }^{10}$ Be proxy data.

It is equally dangerous to evaluate other solar/heliospheric/terrestrial indices from sunspot numbers by extrapolating an empirical relation obtained for the last few decades back in time. This is because the last decades (after the 1950s), which are well covered by direct observations of solar, terrestrial and heliospheric parameters, corresponded to a very high level of solar activity. After a steep rise in activity level between the late 19th and mid 20th centuries, the activity remained at a roughly constant high level, being totally dominated by the 11-year cycle without a long-term trends. Accordingly, all empirical relations built based on data for this period are focused on the 11-year variability and can overlook possible long-term trends (Mursula et al. 2003). This may affect all regression-based reconstructions, whose results 


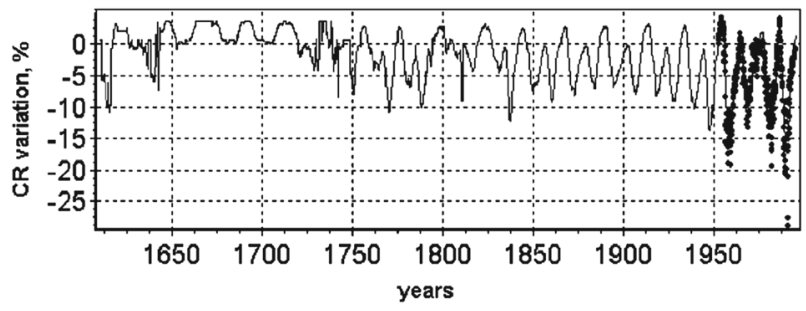

Fig. 15 An unsuccessful attempt of the reconstruction of cosmic-ray intensity in the past using a regression with sunspot numbers. Dots represent the observed cosmic-ray intensity since 1951 . Note the absence of a long-term trend. Image reproduced by permission from Belov et al. (2006), copyright by Elsevier

cannot be independently (directly or indirectly) tested. In particular, this may be related to solar irradiance reconstructions, which are often based on regression-like models, built and verified using data for the last three solar cycles, when there was no strong trend in solar activity.

As an example let us consider an attempt (Belov et al. 2006) to reconstruct cosmicray intensity since 1610 from sunspot numbers using a (nonlinear) regression. The regression between the count rate of a neutron monitor and sunspot numbers (Fig. 4), established for the last 50 years is highly significant. Based on that, Belov et al. (2006) extrapolated the regression back in time to produce a reconstruction of cosmic-ray intensity (quantified in NM count rate) to 1560 (see Fig. 15). One can see that there is no notable long-term trend in the reconstruction, and the fact that all CR maxima essentially lie at the same level, from the Maunder minimum to modern times, is noteworthy. It would be difficult to dispute such a result if there was no direct test for $\mathrm{CR}$ levels in the past. Independent reconstructions based on cosmogenic isotopes or theoretical considerations (e.g., Usoskin et al. 2002a; Scherer et al. 2004; Scherer and Fichtner 2004; McCracken and Beer 2007) provide clear evidence that cosmic-ray intensity was essentially higher during the Maunder minimum than nowadays. This example shows how easy it is to overlook an essential feature in a reconstruction based on a regression extrapolated far beyond the period it is based on. Fortunately, for this particular case we do have independent information that can prevent us from making big errors. In many other cases, however, such information does not exist (e.g., for total or spectral solar irradiance), and those who make such unverifiable reconstructions should be careful about the validity of their models beyond the range of the established relations.

\subsubsection{Reconstruction of heliospheric parameters}

The modulation potential $\phi$ (see Sect. 3.1.1) is directly related to cosmogenic isotope production in the atmosphere. It is a parameter describing the spectrum of galactic cosmic rays (see the definition and full description of this index in Usoskin et al. 2005) in the force-field approximation and is sometimes used as a stand-alone index of solar (or, actually, heliospheric) activity. We note that, provided the isotope production rate $Q$ is estimated and geomagnetic changes can be properly accounted for, it is 


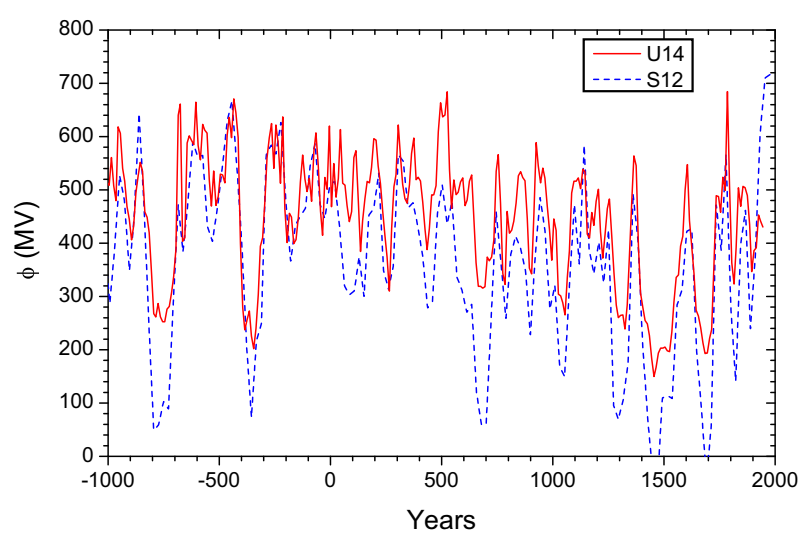

Fig. 16 Two reconstructions of the smoothed modulation potential $\phi$ for the last few decades: 22-year smoothed reconstruction (Steinhilber et al. 2012) based on a composition of ${ }^{14} \mathrm{C}$ and ${ }^{10} \mathrm{Be}$ data (blue dotted curve) and a 10-year averaged reconstruction (Usoskin et al. 2014) based on ${ }^{14} \mathrm{C}$ data (red curve). Error bars (roughly 50-100 MV for each curve) are not shown

straightforward to obtain a time series of the modulation potential, using, e.g., the relation shown in Fig. 10.

Several reconstructions of modulation potential for the last few millenia are shown in Fig. 16. While being quite consistent in the relative changes, they differ in the absolute level and fine details, mostly because of the ambiguity of the exact value of the modulation potential (see discussion in Sect. 3.1.1).

Reconstructions of solar activity often end at this point, representing solar activity by the modulation potential, as some authors (e.g., Beer et al. 2003; Vonmoos et al. 2006; Muscheler et al. 2007) believe that further steps (see Sect. 3.5.3) may introduce additional uncertainties. However, since $\phi$ is a heliospheric, rather than solar, index, the same uncertainties remain when using it as an index of solar activity. Moreover, the modulation potential is a model-dependent quantity (see discussion in Sect. 3.1.1) and therefore does not provide an unambiguous measure of heliospheric activity. In addition, the modulation potential is not a physical index but rather a formal fitting parameter to describe the GCR spectrum near Earth and, thus, is not a universal solaractivity index.

Modulation of GCR in the heliosphere (see Sect. 3.1.1) is mostly defined by the turbulent heliospheric magnetic field (HMF), which ultimately originates from the sun and is thus related to solar activity. It has been shown, using a theoretical model of the heliospheric transport of cosmic rays (e.g., Usoskin et al. 2002a), that on the long-term scale (beyond the 11-year solar cycle) the modulation potential $\phi$ is closely related to the open solar magnetic flux $F_{o}$, which is a physical quantity describing the solar magnetic variability (e.g., Solanki et al. 2000; Krivova et al. 2007).

Sometimes, instead of the open magnetic flux, the mean HMF intensity at Earth orbit, $B$, is used as a heliospheric index (Caballero-Lopez and Moraal 2004; McCracken 2007; Steinhilber et al. 2010). Note that $B$ is linearly related to $F_{o}$ assuming constant solar-wind speed, which is valid on long-term scales. An example of HMF reconstruction for the last 600 years is shown in Fig. 17. In addition, the count rate of 


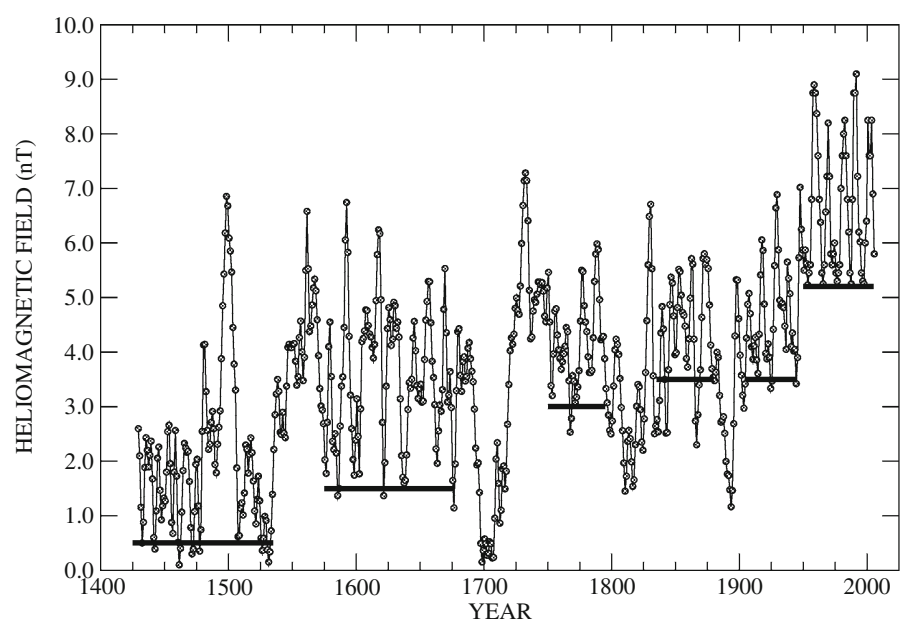

Fig. 17 An example of reconstruction of the heliospheric magnetic field at Earth orbit for the last 600 years from ${ }^{10} \mathrm{Be}$ data. Image reproduced by permission from McCracken (2007), copyright by AGU

a "pseudo" neutron monitor (i.e., a count rate of a neutron monitor if it was operated in the past) is considered as a solar/heliospheric index (e.g., Beer 2000; McCracken and Beer 2007).

\subsubsection{A link to sunspot numbers}

The open solar magnetic flux $F_{o}$ described above is related to the solar surface magnetic phenomena such as sunspots or faculae. Modern physics-based models allow one to calculate the open solar magnetic flux from data of solar observation, in particular sunspots (Solanki et al. 2000, 2002; Krivova et al. 2007; Owens et al. 2012) or geomagnetic activity indices (Lockwood et al. 2014b). Besides the solar active regions, the model includes ephemeral regions. Although these models are based on physical principals, they contain some unknowns like the decay time of the open flux, which cannot be measured or theoretically calculated and has to be found by means of fitting the model to data. This free parameter has been determined by requiring the model output to reproduce the best available data sets for the last 30 years with the help of a genetic algorithm. Inversion of the model, i.e., the computation of sunspot numbers for given $F_{o}$ values is formally a straightforward solution of a system of linear differential equations, however, the presence of noise in the real data makes it only possible in a numerical-statistical way (see, e.g., Usoskin et al. 2004, 2007). By inverting this model one can compute the sunspot-number series corresponding to the reconstructed open flux, thus forging the final link in a chain quantitatively connecting solar activity to the measured cosmogenic isotope abundance. A sunspot-number series reconstructed for the Holocene using ${ }^{14} \mathrm{C}$ isotope data is shown in Fig. 18.

As very important for the climate research, the variations of the total solar irradiance (TSI) are sometimes reconstructed from the solar proxy data (Steinhilber et al. 2009; 


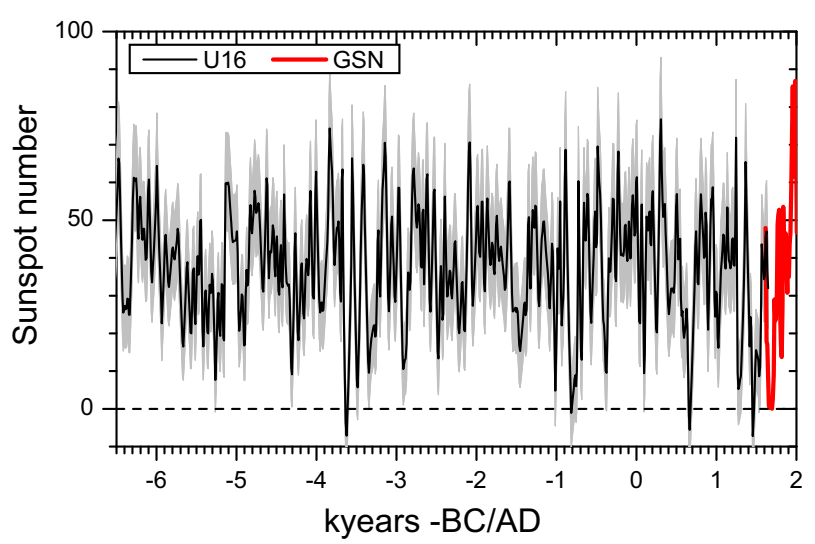

Fig. 18 Long-term sunspot-number reconstruction from ${ }^{14} \mathrm{C}$ data with error bars (after Usoskin et al. 2016a). Group sunspot numbers (Hoyt and Schatten 1998) are shown after 1610

Vieira et al. 2011). However, the absolute range of the TSI variability on the centennialmillennial time scales still remains unknown (Schmidt et al. 2012).

\subsection{Solar activity reconstructions}

Detailed computational models of cosmogenic isotope production in the atmosphere (e.g., Masarik and Beer 1999) have opened up a new possibility for long-term solaractivity reconstruction (e.g., Beer 2000). The first quantitative reconstructions of solar activity from cosmogenic proxy appeared in the early 2000 s based on ${ }^{10} \mathrm{Be}$ deposited in polar ice (Beer et al. 2003; Usoskin et al. 2003c).

Beer et al. (2003) reconstructed the modulation potential on a multi-millennial timescale using the model computations by Masarik and Beer (1999) and the ${ }^{10} \mathrm{Be}$ data from the GISP2 core in Greenland. This result has been extended, even including the ${ }^{14} \mathrm{C}$ data set, and presently covers the whole Holocene (Vonmoos et al. 2006; Steinhilber et al. 2010, 2012). Usoskin et al. (2003c) presented a reconstruction of sunspot activity over the last millennium, based on ${ }^{10} \mathrm{Be}$ data from both Greenland and Antarctica, using a physics-based model described in detail in Usoskin et al. (2004). This result reproduces the four known grand minima of solar activity-Maunder, Spörer, Wolf and Oort minima (see Sect. 4.2). Later Solanki et al. (2004) reconstructed 10 -year-averaged sunspot numbers from the ${ }^{14} \mathrm{C}$ content in tree rings throughout the Holocene and estimated its uncertainties. This result was disputed by Muscheler et al. (2005), whose concurrent model, however, rested on an erroneous normalization, as argued in Solanki et al. (2005). A full revision of the benchmark reconstruction (Solanki et al. 2004), using newer paleomagnetic data, an updated open solar flux model Krivova et al. (2007), and a revised radiocarbon production model (Kovaltsov et al. 2012), has made recently (Usoskin et al. 2014, 2016a). The most recent ${ }^{14} \mathrm{C}$-based reconstruction of solar activity is shown in Fig. 18.

Reconstruction of the HMF from ${ }^{10} \mathrm{Be}$ data has been performed by Caballero-Lopez and Moraal (2004), using a model of CR modulation in the heliosphere and a ${ }^{10} \mathrm{Be}$ 
production model by Webber and Higbie (2003). It was revised (McCracken 2007) to present a detailed reconstruction of HMF intensity since 1428. A recent reconstruction of the heliospheric modulation potential was done by Steinhilber et al. (2012) using the combined principal component analysis of several data sets.

The obtained results are discussed in Sect. 4.

\subsection{Verification of reconstructions}

Because of the diversity of the methods and results of solar-activity reconstruction, it is vitally important to verify them. Even though a full verification is not possible, there are different means of indirect or partial verification, as discussed below. Several solar-activity reconstructions on the millennium timescale, which differ from each other to some degree and are based on terrestrial cosmogenic isotope data, have been published by various groups. Also, they may suffer from systematic effects. Therefore, there is a need for an independent method to verify/calibrate these results in order to provide a reliable quantitative estimate of the level of solar activity in the past, prior to the era of direct observations.

\subsubsection{Comparison with direct data}

The most direct verification of solar-activity reconstruction is a comparison with the actual GSN sunspot data for the last few centuries. However, regression-based models (see Sect. 3.5.1) cannot be tested in this way, since it would require a long set of independent direct data outside the "training" interval. It is usual to include all available data into the "training" period to increase the statistics of the regression, which rules out the possibility of testing the model. On the other hand, such a comparison to the actual GSN since 1610 can be regarded as a direct test for a physics-based model since it does not include phenomenological links over the same time interval. The period of the last four centuries is pretty good for testing purposes since it includes the whole range of solar activity levels from the nearly spotless Maunder minimum to the modern period of a very active sun. However, because of the uncertainties in the sunspot number series (see Sect. 2.2.1), this method shows only an approximate agreement, and direct sunspot numbers cannot serve as the ultimate basis to verify the cosmogenic-based reconstructions. On the contrary, the latter can be used to verify the sunspot number data.

Models focused on the reconstruction of heliospheric parameters (HMF or the modulation potential $\phi$ ) cannot be verified in this manner since no heliospheric data exists before the middle of the twentieth century. Comparison to direct cosmic-ray data after the 1950s (or, with caveats, after the 1930s-McCracken and Beer 2007) is less conclusive, since the latter are of shorter length and correspond to a period of high solar activity, leading to larger uncertainties during grand minima. Moreover, ${ }^{14} \mathrm{C}$ data cannot be tested in this way because of the anthropogenic (Suess) effect and nuclear tests (Sect. 3.2.4).

It is important that some (semi)empirical relations forming the basis for the proxy method are established for the recent decades of high solar activity. The end of the 
Modern grand maximum of activity and the current moderate level of activity, characterized by the highest ever observed cosmic ray flux as recorded by ground-based neutron monitors, the very low level of the HMF and geomagnetic activity, should help to verify the connections between solar activity, cosmic ray fluxes, geomagnetic activity, the heliospheric magnetic field, and open field. Since some of these connections are somewhat controversial, these extreme conditions should help to quantify them better.

\subsubsection{Meteorites and lunar rocks: A direct probe of the galactic cosmic-ray flux}

Another more-or-less direct test of solar/heliospheric activity in the past comes from cosmogenic isotopes measured in lunar rock or meteorites. Cosmogenic isotopes, produced in meteoritic or lunar rocks during their exposure to CR in interplanetary space, provide a direct measure of cosmic-ray flux. Uncertainties due to imprecisely known terrestrial processes, including the geomagnetic shielding and redistribution process, are naturally avoided in this case, since the nuclides are directly produced by cosmic rays in the body of the rock, where they remain until they are measured, without any transport or redistribution. The activity of a cosmogenic isotope in meteorite/lunar rock corresponds to an integral of the balance between the isotope's production and decay, thus representing the time-integrated CR flux over a period determined by the mean life of the radioisotope. The results of different analyses of measurements of cosmogenic isotopes in meteoritic and lunar rocks show that the average GCR flux remained roughly constant-within $10 \%$ over the last million years and within a factor of 1.5 for longer periods of up to $10^{9}$ years (e.g., Vogt et al. 1990; Grieder 2001).

By means of measuring the abundance of relatively short-lived cosmogenic isotopes in meteorites, which fell through the ages, one can evaluate the variability of the CR flux, since the production of cosmogenic isotopes ceases after the fall of the meteorite. A nearly ideal isotope for studying centurial-scale variability is ${ }^{44} \mathrm{Ti}$ with a half-life of $59.2 \pm 0.6$ years (a lifetime of about 85 years). The isotope is produced in nuclear interactions of energetic CR with nuclei of iron and nickel in the body of a meteorite (Bonino et al. 1995; Taricco et al. 2006). Because of its mean life, ${ }^{44} \mathrm{Ti}$ is relatively insensitive to variations in cosmic-ray flux on decadal (11-year Schwabe cycle) or shorter timescales, but is very sensitive to the level of CR flux and its variations on a centurial scale. Using a full model of ${ }^{44} \mathrm{Ti}$ production in a stony meteorite (Michel and Neumann 1998) and data on the measured activity of cosmogenic isotope ${ }^{44} \mathrm{Ti}$ in meteorites, which fell during the past 235 years (Taricco et al. 2006), provides a method to test, in a straightforward manner, reconstructions of solar activity after the Maunder minimum. First, the expected ${ }^{44} \mathrm{Ti}$ activity needs to be calculated from the reconstructed series using the modulation potential, and then compared with the results of actual measurements (see Fig. 19). Since the life-time of the ${ }^{44} \mathrm{Ti}$ is much longer than the 11-year cycle, this method does not allow for the reconstruction of solar/heliospheric activity, but it serves as a direct way to test existing reconstructions independently. As shown by Usoskin et al. (2006c), the ${ }^{44} \mathrm{Ti}$ data confirms significant secular variations of the solar magnetic flux during the last century (cf. Lockwood et al. 1999; Solanki et al. 2000; Wang et al. 2005). Moreover, the recent sunspot 


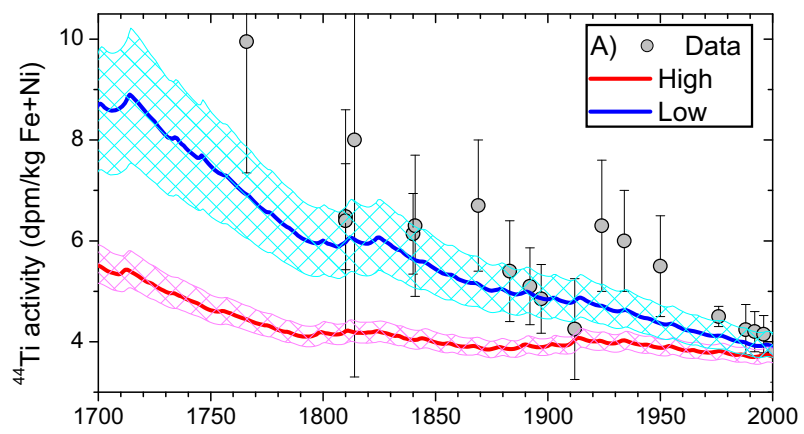

Fig. 19 Time profile of the ${ }^{44}$ Ti activity measured (grey dots with error bars) in the meteorites fallen during the last 250 years (Taricco et al. 2006). The red and blue colored curves with the $1 \sigma$ model uncertainties (hatched areas) depict the modelled ${ }^{44} \mathrm{Ti}$ activity computed for "high" (e.g., Svalgaard and Schatten 2016) and "low" (e.g., Hoyt and Schatten 1998; Usoskin et al. 2016b) reconstructions of solar activity, respectively. Modified after Asvestari et al. (2017)

number reconstructions yielding high solar activity during the 17th and 18th appear inconsistent with the data of ${ }^{44} \mathrm{Ti}$ in meteorites (Fig. 19).

\subsubsection{Comparison between isotopes}

As an indirect test of the solar-activity reconstruction, one can compare different isotopes. The idea behind this test is that two isotopes, ${ }^{14} \mathrm{C}$ and ${ }^{10} \mathrm{Be}$, have essentially different terrestrial fates, so that only the production signal, namely, solar modulation of cosmic rays, can be regarded as common in the two series. Processes of transport/deposition are different (moreover, the ${ }^{14} \mathrm{C}$ series is obtained as an average of the world-wide-distributed samples). The effect of changing geomagnetic fields is also different (although not completely) for the two isotopes, since radiocarbon is globally mixed, while ${ }^{10} \mathrm{Be}$ is only partly mixed before being stored in an archive. Even comparison between data of the same ${ }^{10} \mathrm{Be}$ isotope, but measured in far-spaced ice cores (e.g., Greenland and Antarctica), may help in separating climatic and extraterrestrial factors, since meteorology in the two opposite polar areas is quite different.

The first thorough consistent comparison between ${ }^{10} \mathrm{Be}$ and ${ }^{14} \mathrm{C}$ records for the last millennium was performed by Bard et al. (1997). They assumed that the measured ${ }^{10} \mathrm{Be}$ concentration in Antarctica is directly related to $\mathrm{CR}$ variations. Accordingly, ${ }^{14} \mathrm{C}$ production was considered as proportional to ${ }^{10} \mathrm{Be}$ data. Then, applying a $12-$ box carbon-cycle model, Bard et al. (1997) computed the expected $\Delta^{14} \mathrm{C}$ synthetic record. Finally, these ${ }^{10} \mathrm{Be}$-based $\Delta^{14} \mathrm{C}$ variations were compared with the actual measurements of $\Delta^{14} \mathrm{C}$ in tree rings, which depicted a close agreement in the profile of temporal variation (coefficient of linear correlation $r=0.81$ with exact phasing). Despite some fine discrepancies, which can indicate periods of climatic influence in either (or both) of the series, that result has clearly proven the dominance of solar modulation of cosmogenic nuclide production variations during the last millennium. This conclusion has been confirmed (e.g., Usoskin et al. 2003c; Muscheler et al. 2007) in the sense that quantitative solar-activity reconstructions, based on ${ }^{10} \mathrm{Be}$ and ${ }^{14} \mathrm{C}$ data 
series for the last millennium, yield very similar results, which differ only in small details. However, a longer comparison over the entire Holocene timescale suggests that, while centennial variations of solar activity reconstructed from the two isotopes are very close to each other, there might be a discrepancy in the very long-term trend (Vonmoos et al. 2006; Inceoglu et al. 2015; Usoskin et al. 2016a), whose nature is not clear (climate changes, geomagnetic effects or model uncertainties).

Recently, Usoskin et al. (2009b) studied the dominance of the solar signal in different cosmogenic isotope data on different time scales. They compared the expected ${ }^{10} \mathrm{Be}$ variations computed from ${ }^{14} \mathrm{C}$-based reconstruction of cosmic ray intensity with the actually measured ${ }^{10} \mathrm{Be}$ abundance at the sites and found that: (1) There is good agreement between the ${ }^{14} \mathrm{C}$ and ${ }^{10} \mathrm{Be}$ data sets, on different timescales and at different locations, confirming the existence of a common solar signal in both isotope data; (2) The ${ }^{10} \mathrm{Be}$ data are driven by the solar signal on timescales from about centennial to millennial time scales; (3) The synchronization is lost on short $(<100$ years) timescales, either due to local climate or chronological uncertainties (Delaygue and Bard 2011) but the solar signal becomes important even at short scales during periods of Grand minima of solar activity, (4) There is an indication of a possible systematic uncertainty in the early Holocene (cf. Vonmoos et al. 2006; Inceoglu et al. 2015; Usoskin et al. 2016a), likely due to a not-perfectly-stable thermohaline circulation. Overall, both ${ }^{14} \mathrm{C}$ - and ${ }^{10} \mathrm{Be}$-based records are consistent with each other over a wide range of timescales and time intervals.

Thus, comparison of the results obtained from different sources implies that the variations of cosmogenic nuclides on the long-term scale (centuries to millennia) during the Holocene are primarily defined by the solar modulation of CR.

\subsection{Composite reconstruction}

Most of the earlier solar activity reconstructions are based on single proxy records, either ${ }^{14} \mathrm{C}$ or ${ }^{10} \mathrm{Be}$. Although they are dominated by the same production signal, viz. solar activity, (see Sect. 3.7.3), they still contain essential fractions of noise.

A promising first step in the direction of extracting the common solar signal from different proxy records was made by Steinhilber et al. (2012) who combined, in a composite reconstruction, different ${ }^{10} \mathrm{Be}$ ice core records from Greenland and Antarctica with the global ${ }^{14} \mathrm{C}$ tree ring record. The composite was made in a mathematical way, using the principal component analysis as a numerical tool. This analysis formally finds the common variability in different series, that is assumed to be the solar signal. However, since the used mathematical tool can only work with the relative variability, the reconstruction also yields the relative values rather than absolute values, and it is not available in the terms of sunspot numbers. A particular problem with the composite series is related to the dating uncertainty of ${ }^{10} \mathrm{Be}$. While ${ }^{14} \mathrm{C}$ data are 'absolutely' dated via dendrochronology, the uncertainties in the ice core dating make the ${ }^{10} \mathrm{Be}$ series loose by up to 80 years in the earlier Holocene (Adolphi and Muscheler 2016). Accordingly, the series should be either heavily smoothed, as done by Steinhilber et al. (2012) or corrected for the dating errors, before applying a composite analysis. 
A full physics-based multi-proxy composite reconstruction of the solar activity on the millennial time scale is still pending.

\subsection{Summary}

In this section, a proxy method of past-solar-activity reconstruction is described in detail.

This method is based on the use of indirect proxies of solar activity, i.e., quantitative parameters, which can be measured now, but represent signatures, stored in natural archives, of the different effects of solar magnetic activity in the past. Such traceable signatures can be related to nuclear or chemical effects caused by cosmic rays in the Earth's atmosphere, lunar rocks or meteorites. This approach allows one to obtain homogeneous data sets with stable quality and to improve the quality of data when new measurement techniques become available. It provides the only possible regular indicator of solar activity on a very long-term scale.

The most common proxy of solar activity is formed by data of the cosmogenic radionuclides, ${ }^{10} \mathrm{Be}$ and ${ }^{14} \mathrm{C}$, produced by cosmic rays in the Earth's atmosphere. After a complicated transport in the atmosphere, these cosmogenic isotopes are stored in natural archives such as polar ice, trees, marine sediments, from where they can now be measured. This process is also affected by changes in the geomagnetic field and the climate.

Radioisotope ${ }^{14} \mathrm{C}$, measured in independently-dated tree rings, forms a very useful proxy for long-term solar-activity variability. It participates in the complicated carbon cycle, which smoothes out spatial and short-term variability of isotope production. For the Holocene period, with its stable climate, it provides a useful tool for studying solar activity in the past. Existing models allow the quantitative conversion between the measured relative abundance of ${ }^{14} \mathrm{C}$ and the production rate in the atmosphere. The use of radiocarbon for earlier periods, the glacial and deglaciation epochs, is limited by severe climate and ocean ventilation changes. Radiocarbon data cannot be used after the end of the nineteenth century because of the Suess effect and atmospheric nuclear tests.

Another solar activity proxy is the cosmogenic ${ }^{10} \mathrm{Be}$ isotope measured in stratified polar ice cores. Atmospheric transport of ${ }^{10} \mathrm{Be}$ is relatively straightforward, but its details are as of yet unresolved, leading to the lack of a reliable quantitative model relating the measured isotope concentration in ice to the atmospheric production. Presently, it is common to assume that the production rate is proportional, with an unknown coefficient, to the measured concentration. However, a newly-developed generation of models, which include 3D atmospheric-circulation models, will hopefully solve this problem soon.

Recently, a new proxy, nitrate concentration measured in an Antarctic ice core, has been proposed for long-term solar activity reconstructions, but it still needs verification and model support.

Modern physics-based models make it possible to build a chain, which quantitatively connects isotope production rate and sunspot activity, including subsequently the GCR flux quantified via the modulation potential, the heliospheric index, quantified via the open solar magnetic flux or the average HMF intensity at the Earth's 
orbit, and finally the sunspot-number series. Presently, all these steps can be made using appropriate models allowing for a full basic quantitative reconstruction of solar activity in the past. The main uncertainties in the solar-activity reconstruction arise from paleo-magnetic models and the overall normalization.

An independent verification of the reconstructions, including direct comparison with sunspot numbers, cosmogenic isotopes in meteorites and the comparison of different models with each other, confirms their veracity in both relative variations and absolute level. It also implies that the variations in cosmogenic nuclides on the longterm scale (centuries to millennia) during the Holocene are primarily defined by the solar modulation of $\mathrm{CR}$.

\section{Variability of solar activity over millennia}

Several reconstructions of solar activity on multi-millennial timescales have been performed recently using physics-based models (see Sect. 3) from measurements of ${ }^{14} \mathrm{C}$ in tree rings and ${ }^{10} \mathrm{Be}$ in polar ice. In this section we discuss the temporal variability of thus-reconstructed solar activity on a longer scale.

Here we consider the ${ }^{14} \mathrm{C}$-based decade reconstruction (Usoskin et al. 2016a) of sunspot numbers (shown in Fig. 20). It is identical to that shown in Fig. 18, but is split into two panels for better visibility and highlights the identified grand minima and maxima of solar activity. This series forms the basis for the forthcoming analysis, while differences related to the use of other reconstructions are discussed.

\subsection{Quasi-periodicities and characteristic times}

Although the variability of solar activity in Fig. 20 looks random at first look, some weak quasi-periodicities can be suggested, as discussed in Sect. 2.4. A simple period-

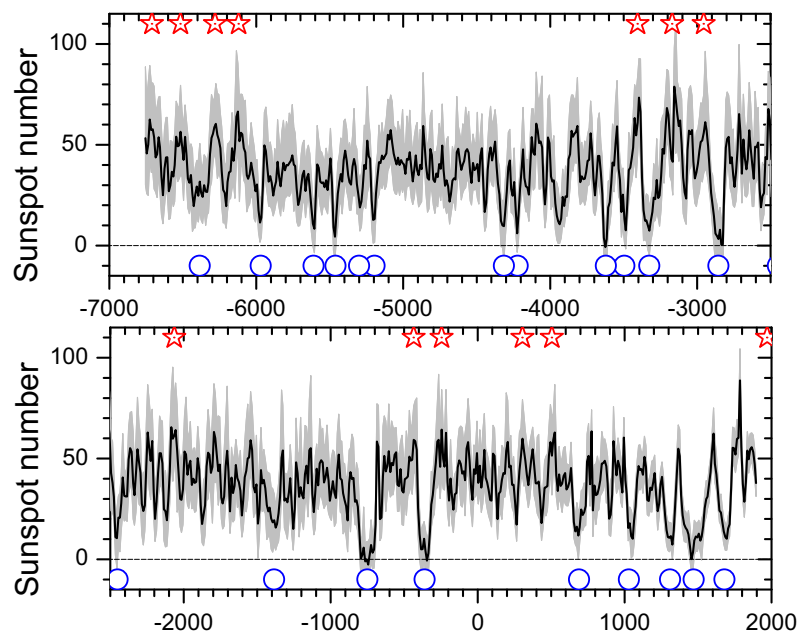

Fig. 20 Sunspot activity (decadal data) throughout the Holocene, reconstructed from ${ }^{14} \mathrm{C}$ by Usoskin et al. (2016a). Blue circles and red stars denote grand minima and maxima, respectively 
icity analysis yields several peaks in the range of periods between 80 and 150 years (cf., Peristykh and Damon 2003), corresponding to the frequency band of the Gleissberg cycle (Ogurtsov et al. 2002). The de Vries/Suess cycle, with a period of about 210 years, forms a prominent in the power spectrum, but it is intermittent and tends to become strong with around 2400 clustering time (Usoskin and Kovaltsov 2004). Another variation with a period of around 350 years can be observed after $6000 \mathrm{BC}$ (cf. Steinhilber et al. 2012). Variations with a characteristic time of 600-700 years are intermittent and can be hardly regarded as a typical feature of solar activity. There is also a weak millennial quasi-periodicity with the period of 1000-1200 years called Eddy cycle (Steinhilber et al. 2012). Of special interest is the 2000-2400 year Hallstatt cycle (see, e.g., Vitinsky et al. 1986; Damon and Sonett 1991; Vasiliev and Dergachev 2002), which is relatively stable and mostly manifests itself as a modulation of longterm solar activity, leading to the clustering of grand minima (Usoskin et al. 2016a). Although its possible terrestrial origin (geomagnetic or climate) was discussed earlier (Vasiliev and Dergachev 2002; Vasiliev et al. 2012), it is shown by Usoskin et al. (2016a) to have solar origin. In particular, grand minima and maxima tend to cluster around highs and lows of the Hallstatt cycle.

\subsection{Grand minima of solar activity}

A very particular type of solar activity is the grand minimum, when solar activity is greatly reduced. The most famous is the Maunder minimum in the late seventeenth century, which is discussed below in some detail (for details see Soon and Yaskell 2003; Usoskin et al. 2015). Grand minima are believed to correspond to a special state of the dynamo (Sokoloff 2004; Miyahara et al. 2006b; Moss et al. 2008; Käpylä et al. 2016), and its very existence poses a challenge for the solar-dynamo theory. It is noteworthy that dynamo models do not agree on how often such episodes occur in the sun's history and whether their appearance is regular or random. For example, the commonly used mean-field dynamo yields a fairly-regular 11-year cycle (Charbonneau 2010), while dynamo models including a stochastic driver predict the intermittency of solar magnetic activity (Choudhuri 1992; Schüssler et al. 1994; Schmitt et al. 1996; Ossendrijver 2000; Weiss and Tobias 2000; Mininni et al. 2001; Charbonneau 2001). Most of the models predict purely random occurrence of the grand minima, without any intrinsic long-term memory (Moss et al. 2008). Although cosmogenic isotope data suggest the possible existence of such memory (Usoskin et al. 2007), statistics is not sufficient to distinguish between the two cases (Usoskin et al. 2009d).

\subsubsection{The Maunder minimum}

The Maunder minimum (MM) is a representative of grand minima in solar activity (e.g., Eddy 1976), when sunspots have almost completely vanished from the solar surface, while the solar wind kept blowing, although at a reduced pace (Cliver et al. 1998; Usoskin et al. 2001b). As proposed by Lockwood and Owens (2014), the solar wind was uniform and slow, 250-275 km/s, nearly half of the modern time velocity. There is some uncertainty in the definition of the duration of MM: the "formal" duration 
is 1645-1715 (Eddy 1976), while its deep phase with the absence of apparent sunspot cyclic activity is often considered as 1645-1700, with the low, but very clear, solar cycle of 1700-1712 being ascribed to a recovery or transition phase (Usoskin et al. 2000). MM was amazingly well covered by direct sunspot observations (Hoyt and Schatten 1996) - more than $95 \%$ of days have formal records (however many of them are generic) and 30-50\% of days have explicit data (Vaquero et al. 2015). The late part of MM after the 1680s is particularly weel covered with direct data from the French school of astronomy (Ribes and Nesme-Ribes 1993). On the other hand, sunspots appeared rarely (during $\sim 2 \%$ of the days) and seemingly sporadically, without an indication of the 11-year cycle.

Some recent studies suggested that the sunspot activity level might have been underestimated during MM: Zolotova and Ponyavin (2015) proposed that the annual number of sunspot groups was as high as 3-8 (the sunspot number 50-100) during MM, Svalgaard and Schatten (2016) proposed much more modest peak annual number of sunspot groups as 2-3 (25-40 in sunspot number) but still too high. These claims were based on the fact that original data include many generic statements of the absence of sunspots for long periods of time, and should be dismissed. However, these statements made by professional astronomers in a dedicated monitoring of the sun, should be considered seriously. A thorough analysis of all the available sunspot data made by applying 'filters' of different degrees of strictness was made by Vaquero et al. (2015) who concluded that the level of sunspot activity was indeed very low during MM, even if considering only explicit records. The low level of activity during MM was confirmed also by an aggregate study of other indirect data for that period (Usoskin et al. 2015): while there are known auroral observations during MM, they all are limited to high latitudes (close to the auroral oval), where polar lights occur even without strong geomagnetic storms; data of cosmogenic isotopes ${ }^{14} \mathrm{C}$ measured in tree trunks and ${ }^{44} \mathrm{Ti}$ in fallen meteorites clearly indicate a very high flux of cosmic rays (low solar activity) during MM.

Such low level of activity makes it almost impossible to apply standard methods of time-series analysis to sunspot data during MM (e.g., Frick et al. 1997). Therefore, special methods such as the distribution of spotless days versus days with sunspots (e.g., Harvey and White 1999; Kovaltsov et al. 2004; Vaquero et al. 2014) or an analysis of sparsely-occurring events (Usoskin et al. 2000) should be applied in this case. Using these methods, Usoskin et al. (2001b) have shown that sunspot occurrence during the Maunder minimum was gathered into two large clusters (1652-1662 and 1672-1689), with the mass centers of these clusters being in 1658 and 1679-1680. Together with the sunspot maxima before (1640) and after (1705) the deep Maunder minimum, this implies a dominant 22-year periodicity in sunspot activity throughout the Maunder minimum (Mursula et al. 2001), with a subdominant 11-year cycle emerging towards the end of the Maunder minimum (Ribes and Nesme-Ribes 1993; Mendoza 1997; Usoskin et al. 2000; Vaquero et al. 2015) and becoming dominant again after 1700. Similar behavior of a dominant 22-year cycle and a weak subdominant Schwabe cycle during the Maunder minimum has been found in other indirect solar proxy data: auroral occurrence (Křivský and Pejml 1988; Schlamminger 1990; Silverman 1992) and ${ }^{14} \mathrm{C}$ data (Stuiver and Braziunas 1993; Kocharov et al. 1995; Peristykh and Damon 1998; Miyahara et al. 2006b). This is in general agreement with the concept of "immersion" 
of 11-year cycles during the Maunder minimum (Vitinsky et al. 1986, and references therein). This concept means that full cycles cannot be resolved and sunspot activity only appears as pulses around cycle-maximum times.

An analysis of ${ }^{10} \mathrm{Be}$ data (Beer et al. 1998) implied that the 11-year cycle was weak but fairly regular during the Maunder minimum, but its phase was inverted (Usoskin et al. 2001b). A recent theoretical study (Owens et al. 2012; Wang and Sheeley 2012) confirms that such a phase change between cosmic rays and solar activity can indeed appear for very weak cycles.

Until recently, it was believed (Vitinsky et al. 1986; Ribes and Nesme-Ribes 1993; Sokoloff and Nesme-Ribes 1994; Usoskin et al. 2000, 2001b; Miyahara et al. 2006b) that transition from the normal high activity to the deep minimum did not have any apparent precursor before MM. However, newly recovered data suggest that the start of the Maunder minimum might had been not very sudden but via a regular cycle of reduced height (Vaquero et al. 2011). A 22-year cycle was dominant in sunspot occurrence during the deep minimum (1645-1700), with the subdominant 11-year cycle, which became visible only in the late phase of the Maunder minimum. There is an indication that the length of solar cycle may slightly extend during and already slightly before a grand minimum (Miyahara et al. 2004; Nagaya et al. 2012), which is in agreement (note that the possible cycle maximum in 1650 discussed there was based on an erroneous data point and should be dismissed) with the results by Vaquero et al. (2015).

The 11-year Schwabe cycle started dominating solar activity after 1700. Recovery of sunspot activity from the deep minimum to normal activity was gradual, passing through a period of nearly-linear amplification of the 11-year cycle.

Although the Maunder minimum is the only one with available direct sunspot observations, its predecessor, the Spörer minimum from 1450-1550, is covered by precise bi-annual measurements of ${ }^{14} \mathrm{C}$ (Miyahara et al. 2006a). An analysis of this data (Miyahara et al. 2006a, b) reveals a similar pattern with the dominant 22-year cycle and suppressed 11-year cycle, thus supporting the idea that the above general scenario may be typical for a grand minimum. A similar pattern has been recently also for an un-named grand minima in the fourth century BC (Nagaya et al. 2012).

A very important feature of sunspot activity during the Maunder minimum was its strong north-south asymmetry, as sunspots were only observed in the southern solar hemisphere during the end of the Maunder minimum (Ribes and Nesme-Ribes 1993; Sokoloff and Nesme-Ribes 1994). This observational fact has led to intensive theoretical efforts to explain a significant asymmetry of the sun's surface magnetic field in the framework of the dynamo concept (see the review by Sokoloff 2004, and references therein). Note that a recent discovery (Arlt 2008, 2009) of the Staudacher's original drawings of sunspots in late eighteenth century shows that similarly asymmetric sunspot occurrence existed also in the beginning of the Dalton minimum in 1790s (Usoskin et al. 2009c). However, the northern hemisphere dominated at that period contrary to the situation during the Maunder minimum.

\subsubsection{Grand minima on a multi-millennial timescale}

The presence of grand minima in solar activity on the long-term scale has been mentioned numerously (e.g., Eddy 1977b; Solanki et al. 2004; Usoskin et al. 2007; 
Steinhilber et al. 2012; Inceoglu et al. 2015), using the radioisotope data of ${ }^{14} \mathrm{C}$ in tree rings and ${ }^{10} \mathrm{Be}$ in ice cores. For example, Eddy (1977a) identified major excursions in the detrended ${ }^{14} \mathrm{C}$ record as grand minima and maxima of solar activity and presented a list of six grand minima and five grand maxima for the last 5000 years (see Table 2). Stuiver and Braziunas (1989) and Stuiver et al. (1991) also studied grand minima as systematic excesses of the high-pass filtered ${ }^{14} \mathrm{C}$ data and suggested that the minima are generally of two distinct types: short minima of duration 50-80 years (called Maunder-type) and longer minima collectively called Spörer-like minima. Using the same method of identifying grand minima as significant peaks in high-pass filtered $\Delta^{14} \mathrm{C}$ series, Voss et al. (1996) provided a list of 29 such events for the past 8000 years. A similar analysis of bumps in the ${ }^{14} \mathrm{C}$ production rate was presented recently by Goslar (2003). However, such studies retained a qualitative element, since they are based on high-pass-filtere ${ }^{14} \mathrm{C}$ data and thus implicitly assume that ${ }^{14} \mathrm{C}$ variability can be divided into short-term solar variations and long-term changes attributed solely to the slowly-changing geomagnetic field. This method ignores any possible long-term changes in solar activity on timescales longer than 500 years (Voss et al. 1996). The modern approach, based on physics-based modelling (Sect. 3), allows for the quantitative reconstruction of the solar activity level in the past, and thus, for a more realistic definition of the periods of grand minima or maxima.

A list of 25 grand minima, identified in the quantitative solar-activity reconstruction of the last 11000 years, shown in Fig. 20, is presented in Table 2 (after Usoskin et al. 2007, 2016a). The cumulative duration of the grand minima is about 1900 years, indicating that the sun in its present evolutionary stage spends $\sim^{1 / 6}(17 \%)$ of its time in a quiet state, corresponding to grand minima. Note that the definition of grand minima is quite robust.

It was shown by Usoskin et al. (2014), using ${ }^{14} \mathrm{C}$ data for the last three millennia, that grand minima correspond to a special mode of the solar dynamo which is clearly separated from the main mode of moderate activity. The probability density function (PDF) of the occurrence of decadal sunspot numbers in the reconstruction based on ${ }^{14} \mathrm{C}$ for the last three millennia (Usoskin et al. 2014) is shown in Fig. 21. One can see that the PDF has a clear bimodal structure, where the main mode corresponds to the general mode of moderate activity (20-60 in decadal sunspot numbers), while the secondary maximum represents a statistically different mode of low activity (decadal sunspot numbers below 20) corresponding to Grand minima.

The question of whether the occurrence of grand minima in solar activity is a regular or chaotic process is important for understanding the action of the solar-dynamo machine. Even a simple deterministic numerical dynamo model can produce events comparable with grand minima (Brandenburg et al. 1989; Käpylä et al. 2016). Such models can also simulate a sequence of grand minima occurrences, which are irregular and seemingly chaotic (e.g., Jennings and Weiss 1991; Tobias et al. 1995; Covas et al. 1998). The presence of long-term dynamics in the dynamo process is often explained in terms of the $\alpha$-effect, which, being a result of the electromotive force averaged over turbulent vortices, can contain a fluctuating part (e.g., Hoyng 1993; Ossendrijver et al. 1996) leading to irregularly occurring grand minima (e.g., Brandenburg and Spiegel 2008). The present dynamo models can reproduce almost all the observed 
Table 2 Conservative list with approximate dates (in -BC/AD) of grand minima in reconstructed solar activity [1-listed in Usoskin et al. (2007); 2-listed in Inceoglu et al. (2015); 3-listed in Usoskin et al. (2016a)]

\begin{tabular}{|c|c|c|}
\hline Center (-BC/AD) & Duration (years) & Comment \\
\hline 1680 & 80 & Maunder $^{a}$ \\
\hline 1470 & 160 & Spörer \\
\hline 1310 & 80 & Wolf \\
\hline 1030 & 80 & Oort \\
\hline 690 & 80 & $1-3$ \\
\hline-360 & 80 & $1-3$ \\
\hline-750 & 120 & $1-3$ \\
\hline-1385 & 70 & $1-3$ \\
\hline-2450 & 40 & 2,3 \\
\hline-2855 & 90 & $1-3$ \\
\hline-3325 & 90 & $1-3$ \\
\hline-3495 & 50 & $1-3$ \\
\hline-3620 & 50 & $1-3$ \\
\hline-4220 & 30 & $1-3$ \\
\hline-4315 & 50 & $1-3$ \\
\hline-5195 & 50 & 2,3 \\
\hline-5300 & 50 & $1-3$ \\
\hline-5460 & 40 & $1-3$ \\
\hline-5610 & 40 & $1-3$ \\
\hline-6385 & 130 & $1-3$ \\
\hline-7035 & 50 & 1 \\
\hline-7305 & 30 & 1 \\
\hline-7515 & 150 & 1 \\
\hline-8215 & 110 & 1 \\
\hline-9165 & 150 & 1 \\
\hline
\end{tabular}

$a$ independently know

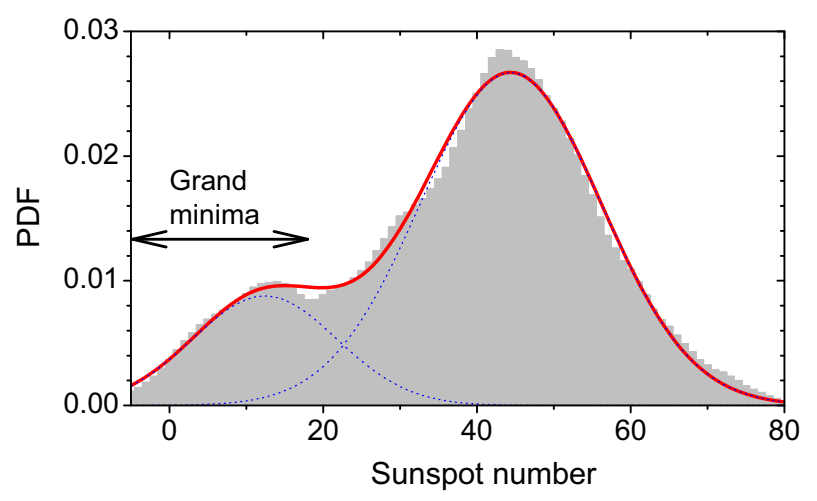

Fig. 21 Probability density function of the reconstructed decadal sunspot numbers for the last three millennia (grey histogram). Shown is also the best-fit bimodal Gaussian distribution (red curve with the two modes shown by dotted blue lines). The Grand minimum mode is indicated by the arrow. (Modified after Usoskin et al. 2014) 
features of the solar cycle under ad hoc assumptions (e.g., Pipin et al. 2012), although it is still unclear what leads to the observed variability. Most of these models predict that the occurrence of grand minima is a purely random "memoryless" Poisson-like process, with the probability of a grand minimum occurring being constant at any given time. This unambiguously leads to the exponential shape of the waiting-time distribution (waiting time is the time interval between subsequent events) for grand minima.

Usoskin et al. (2007) performed a statistical analysis of grand minima occurrence time (Table 2) and concluded that their occurrence is not a result of long-term cyclic variations, but is defined by stochastic/chaotic processes. Moreover, waiting-time distribution deviates from the exponential law. This implies that the event occurrence is still random, but the probability is nonuniform in time and depends on the previous history. In the time series it is observed as a tendency of the events to cluster together with a relatively-short waiting time, while the clusters are separated by long eventfree intervals (cf. Sect. 4.1). Such behavior can be interpreted in different ways, e.g., self-organized criticality or processes related to accumulation and release of energy. This poses a strong observational constraint on theoretical models aiming to explain the long-term evolution of solar activity (Sect. 4.4.1). However, as discussed by Moss et al. (2008) and Usoskin et al. (2009d), the observed feature can be an artefact of the small statistics (only 27 grand minima are identified during the Holocene), making this result only indicative and waiting for a more detailed investigation.

A histogram of the duration of grand minima from Table 2 is shown in Fig. 22. The mean duration is 70 year but the distribution is bimodal. The minima tend to be either of a short (30-90 years) duration similar to the Maunder minimum, or rather long (>100 years), similar to the Spörer minimum, in agreement with earlier conclusions (Stuiver and Braziunas 1989). This suggests that grand minima correspond to a special state of the dynamo. Once falling into a grand minimum as a result of a stochastic/chaotic, but non-Poisson process, the dynamo is "trapped" in this state and its behavior is driven by deterministic intrinsic features.

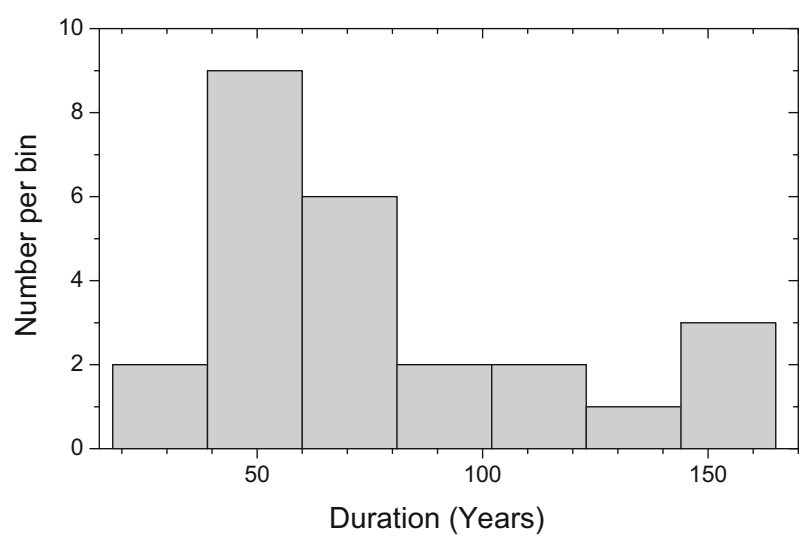

Fig. 22 Histogram of the duration of grand minima from Table 2 


\subsection{Grand maxima of solar activity}

\subsubsection{The modern episode of active sun}

In the last decades we were living in a period of a very active sun with a level of activity that is very high for the last few centuries covered by direct solar observation. The sunspot number was growing rapidly between 1900 and 1940, with more than a doubling average group sunspot number, and has remained at that high level until recently (see Fig. 1). Note that growth comes mostly from raising the cycle maximum amplitude, while sunspot activity always returns to a very low level around solar cycle minima. While the average group sunspot number (using GSN) for the period 17501900 was $35 \pm 9(39 \pm 6$, if the Dalton minimum in 1797-1828 is not counted), it stands high at the level of $75 \pm 3$ for 1950-2000. Therefore, the modern active sun episode, which started in the 1940s, can be regarded as the modern grand maximum of solar activity, as opposed to a grand minimum (Wilson 1988b). As discussed by Clette et al. (2014, see their Figure 65), the number of spotless days during cycles 1222 was half of that for another relatively high activity period ca. 1850. This again suggests the uniqueness of the modern grand maximum on the centennial time scale. The reality of the Modern grand maximum was independently confirmed by Zięba and Nieckarz (2014) who have shown, by studying active versus passive (spotless) days that cycles 17-23 were more active, compared to cycles 8-15.

Although uncertainties in sunspot numbers during the 18th and 19th centuries (see discussion in Sect. 2.2.1) make it a bit unclear on the centennial time scale, data on cosmogenic isotopes (Usoskin et al. 2003c; Solanki et al. 2004; Inceoglu et al. 2015) imply that such high activity episodes occur quite seldom.

However, as we can securely say now, after the very weak solar minimum in 20082009 (e.g., Gibson et al. 2011), solar activity returns to its normal moderate level in cycle \# 24. Thus, the high activity episode known as the Modern grand maximum is over.

Is such high solar activity typical or is it something extraordinary? While it is broadly agreed that the modern active sun episode is a special phenomenon, the question of how (a)typical such upward bumps are from "normal" activity is a topic of hot debate.

\subsubsection{Grand maxima on a multi-millennial timescale}

The question of how often grand maxima occur and how strong they are, cannot be studied using the 400-year-long series of direct observations. An increase in solar activity around $1200 \mathrm{AD}$, also related to the Medieval temperature optimum, is sometimes qualitatively regarded as a grand maximum (Wilson 1988b; de Meyer 1998), but its magnitude is lower than the modern maximum (e.g., Usoskin et al. 2003c). Accordingly, it was not included in a list of grand maxima by Eddy (1977b, a).

A quantitative analysis is only possible using proxy data, especially cosmogenic isotope records. Using a physics-based analysis of solar-activity series reconstructed from ${ }^{10} \mathrm{Be}$ data from polar (Greenland and Antarctica) archives, Usoskin et al. (2003c, 2004) stated that the modern maximum is unique in the last millennium. Then, using a similar analysis of the ${ }^{14} \mathrm{C}$ calibrated series, Solanki et al. (2004) found that the modern 
activity burst is not unique, but a very rare event, with the previous burst occurring about 8 millennia ago. An update (Usoskin et al. 2006a) of this result, using a more precise paleo-magnetic reconstruction by Korte and Constable (2005) since 5000 BC, suggests that an increase of solar activity comparable with the modern episode might have taken place around $2000 \mathrm{BC}$, i.e., around 4 millennia ago, in agreement with more recent studies by Steinhilber et al. (2012) and Inceoglu et al. (2015). On the other hand, the definition of grand maxima is less robust than grand minima and is sensitive to other parameters such as geomagnetic field data or overall normalization (Usoskin et al. 2016a).

Keeping possible uncertainties in mind, let us consider a list of the largest grand maxima (the 50 year smoothed sunspot number stably exceeding 50), identified for the last eleven millennia using cosmogenic isotope data, as shown in Table 3. A total of 23 grand maxima have been identified with a total duration of around 1400 years, suggesting that the sun spends around $12 \%$ of its time in an active state. A statistical analysis of grand-maxima-occurrence time suggests that they do not follow longterm cyclic variations, but a clustering near highs of the Hallstatt cycle is observed (Usoskin et al. 2016a). The distribution of the waiting time between consecutive grand

Table 3 Conservative list with approximate dates (in $-\mathrm{BC} / \mathrm{AD}$ ) of grand maxima in reconstructed solar activity [1-listed in Usoskin et al. (2007); 2-listed in Inceoglu et al. (2015); 3-listed in Usoskin et al. (2016a)]

\begin{tabular}{lcl}
\hline No. & Center & Duration \\
\hline 1970 & 80 & Modern \\
505 & 50 & 2,3 \\
305 & 30 & 2,3 \\
-245 & 70 & 2,3 \\
-435 & 50 & $1-3$ \\
-2065 & 50 & $1-3$ \\
-2955 & 30 & 2,3 \\
-3170 & 100 & $1-3$ \\
-3405 & 50 & 2,3 \\
-3860 & 50 & $1-3$ \\
-6120 & 40 & $1-3$ \\
-6280 & 40 & 2,3 \\
-6515 & 70 & 1 \\
-6710 & 40 & 1 \\
-6865 & 50 & 1 \\
-7215 & 30 & 1 \\
-7660 & 80 & 1 \\
-7780 & 20 & 1 \\
-7850 & 20 & 1 \\
-8030 & 50 & 1 \\
-8350 & 70 & 1 \\
-8915 & 190 & 1 \\
-9375 & 130 & \\
\hline
\end{tabular}


maxima is not unambiguously clear, but also hints at a deviation from exponential law. The duration of grand maxima has a smooth distribution, which nearly exponentially decreases towards longer intervals. Most of the reconstructed grand maxima (about $70 \%$ ) were not longer than 50 years, and only five grand minima (including the modern one) have been longer than 70 years (cf. Barnard et al. 2011). Note, that the Modern grand maximum is over now and we are living during the epoch of moderate or even weak solar activity.

It is still unclear wether grand maxima correspond to a special state of solar dynamo or rather to a tail of the regular mode. Although there are some indications for the former (Usoskin et al. 2016a), they are inconclusive.

\subsection{Related implications}

Reconstructions of long-term solar activity have different implications in related areas of science. The results, discussed in this overview, can be used in such diverse research disciplines as theoretical astrophysics, solar-terrestrial studies, paleo-climatology, and even archeology and geology. We will not discuss all possible implications of longterm solar activity in great detail but only briefly mention them here.

\subsubsection{Theoretical constrains}

The basic principles of the occurrence of the 11-year Schwabe cycle are more-orless understood in terms of the solar dynamo, which acts, in its classical form (e.g., Parker 1955), as follows (see detail in Charbonneau 2010). Differential rotation $\Omega$ produces a toroidal magnetic field from a poloidal one, while the " $\alpha$-effect", associated with the helicity of the velocity field or Joy's Law tilt of active regions, produces a poloidal magnetic field from a toroidal one. This classical model results in a periodic process in the form of propagation of a toroidal field pattern in the latitudinal direction (the "butterfly diagram"). As evident from observation, the solar cycle is far from being a strictly periodic phenomenon, with essential variations in the cycle length and especially in the amplitude, varying dramatically between nearly spotless grand minima and very large values during grand maxima. The mere fact of such great variability, known from sunspot data, forced solar physicists to develop dynamo models further. Simple deterministic numerical dynamo models, developed on the basis of Parker's migratory dynamo, can simulate events, which are seemingly comparable with grand minima/maxima occurrence (e.g., Brandenburg et al. 1989). However, since variations in the solar-activity level, as deduced from cosmogenic isotopes, appear essentially nonperiodic and irregular, appropriate models have been developed to reproduce irregularly-occurring grand minima (e.g., Jennings and Weiss 1991; Tobias et al. 1995; Covas et al. 1998). Models, including an ad hoc stochastic driver (Choudhuri 1992; Schmitt et al. 1996; Ossendrijver 2000; Weiss and Tobias 2000; Mininni et al. 2001; Charbonneau 2001; Charbonneau et al. 2004; Käpylä et al. 2016), are able to reproduce the great variability and intermittency found in the solar cycle (see the review by Charbonneau 2010). A recent statistical result of grand minima occurrence (Sect. 4.3.2) shows disagreement between observational data, depicting a 
degree of self-organization or "memory", and the above dynamo model, which predicts a pure Poisson occurrence rate for grand minima (see Sect. 4.2). This poses a new constraint on the dynamo theory, responsible for long-term solar-activity variations (Sokoloff 2004; Moss et al. 2008).

In general, the following additional constraints can be posed on dynamo models aiming to describe the long-term (during the past 11000 years) evolution of solar magnetic activity.

- The sun spends about $3 / 4$ of its time at moderate magnetic-activity levels, about $1 / 6$ of its time in a grand minimum and about $1 / 5^{-1} / 10$ in a grand maximum. Recent solar activity corresponds to a grand maximum, which has ceased after solar cycle 23.

- Grand minima form a special, statistically significant, mode of solar dynamo. The existence of the Grand maximum mode is hinted but not conclusive.

- Occurrence of grand minima and maxima is not a result of long-term cyclic variations but is defined by stochastic/chaotic processes.

- Observed statistics of the occurrence of grand minima and maxima display deviation from a "memory-less" Poisson-like process, but tend to either cluster events together or produce long event-free periods.

Grand minima and maxima tend to cluster around lows and highs of the $\approx 2400$ year Hallstatt cycle, respectively. This can be interpreted in different ways, such as self-organized criticality (e.g., Carvalho and Prado 2000), a time-dependent Poisson process (e.g., Wheatland 2003), or some memory in the driving process (e.g., Mega et al. 2003).

- Grand minima tend to be of two different types: short minima of Maunder type and long minima of Spörer type. This suggests that a grand minimum is a special state of the dynamo.

- Duration of grand maxima resemble a random Possion-like process, in contrast to grand minima.

\subsubsection{Solar-terrestrial relations}

The sun ultimately defines the climate on Earth supplying it with energy via radiation received by the terrestrial system, but the role of solar variability in climate variations is far from being clear. Solar variability can affect the Earth's environment and climate in different ways (see, e.g., reviews by Haigh 2007; Gray et al. 2010). Variability of total solar irradiance (TSI) measured during recent decades is known to be too small to explain observed climate variations (e.g., Foukal et al. 2006; Fröhlich 2006; Yeo et al. 2014). On the other hand, there are other ways solar variability may affect the climate, e.g., an unknown long-term trend in TSI (Solanki and Krivova 2004; Wang et al. 2005) or a terrestrial amplifier of spectral irradiance variations (Shindell et al. 1999; Haigh et al. 2010). Uncertainties in the TSI/SSI reconstructions remain large (Schmidt et al. 2012; Yeo et al. 2014), making it difficult to assess climate models on the long-term scale. Alternatively, an indirect mechanism also driven by solar activity, such as ionization of the atmosphere by CR (Usoskin and Kovaltsov 2006) or the global terrestrial current system (Tinsley and Zhou 2006) can modify atmospheric properties, 
in particular cloud cover (Ney 1959; Svensmark 1998; Usoskin and Kovaltsov 2008a). Although the role of this direct mechanism is found to be small (Mironova et al. 2015), indirect effects of energetic particles may be still notable (e.g., Gray et al. 2010; Calisto et al. 2011; Martin-Puertas et al. 2012).

Accordingly, improved knowledge of the solar driver's variability may help in disentangling various effects in the very complicated system that is the terrestrial climate (e.g., Gray et al. 2010). It is of particular importance to know the driving forces in the pre-industrial era, when all climate changes were natural. Knowledge of the natural variability can lead to an improved understanding of anthropogenic effects upon the Earth's climate.

Studies of the long-term solar-terrestrial relations are mostly phenomenological, lacking a clear quantitative physical mechanism. Therefore, more precise knowledge of past solar activity, especially since it is accompanied by continuous efforts of the paleo-climatic community on improving climatic data sets, is crucial for improved understanding of the natural (including solar) variability of the terrestrial environment.

\subsection{Summary}

In this section, solar activity on a longer scale is discussed, based on recent reconstructions.

According to these reconstructions, the sun has spent about $70 \%$ of its time during the Holocene, which is ongoing, in a normal state characterized by medium solar activity. About $15-20 \%$ of the time the sun has experienced a grand minimum, while $10-15 \%$ of the time has been taken up by periods of very high activity.

One of the main features of long-term solar activity is its irregular behavior, which cannot be described by a combination of quasi-periodic processes as it includes an essentially random component.

Grand minima, whose representative is the Maunder minimum of the late seventeenth century, are typical solar phenomena. Approximately 25 grand minima can be robustly identified in solar activity reconstructions for the Holocene period. Their occurrence suggests that they appear not periodically, but tend to appear in clusters separated by 2000-2500 years (the Hallstatt cycle), and having a recurrence period of $\approx 210$ years (Suess/de Vries cycle) within the clusters. Grand minima tend to be of two distinct types: short (Maunder-like) and longer (Spörer-like). The appearance of grand minima can be reproduced by modern stochastic-driven dynamo models to some extent, but some problems still remain to be resolved.

The recent level of solar activity (after the 1940s) was very high, corresponding to a grand maximum, which are typical but rare events in solar behavior. However, this grand maximum has ceased after solar cycle 23. The duration of grand maxima resembles a random Possion-like process, in contrast to grand minima.

These observational features of the long-term behavior of solar activity have important implications, especially for the development of theoretical solar-dynamo models and for solar-terrestrial studies. 


\section{Solar energetic particles in the past}

In addition to galactic cosmic rays, which are always present in the Earth's vicinity, sometimes sporadic solar energetic particle (SEP) events with a greatly enhanced flux of less energetic particles in the interplanetary medium also occur (e.g., Klecker et al. 2006). Strong SEP events mostly originate from CME-related shocks propagating in the solar corona and interplanetary medium, that lead to effective bulk acceleration of charged particles (e.g., Cane and Lario 2006). Although these particles are significantly less energetic than GCRs, they can occasionally be accelerated to an energy reaching up to several $\mathrm{GeV}$ or more, which is enough to initiate the atmospheric cascade. Peak intensity of SEP flux can be very high, up to $10^{4}$ particles (with energy $>30 \mathrm{MeV}$ ) per $\mathrm{cm}^{2}$ per second. In fact, the long-term average flux (or fluence) of SEP is mostly defined by rare major events, which occur a few times per solar cycle, with only minor contributions from a large number of weak events (Shea and Smart 1990, 2002). As an example, energy spectra of GCR and SEP are shown in Fig. 23 for the day of January 20, 2005, when an extreme SEP event took place. Such SEPs dominate the low-energy section of cosmic rays (below hundreds of $\mathrm{MeV}$ of a particle's kinetic energy), which is crucial for the radiation environment, and play an important role in solar-terrestrial relations. For many reasons it is important to know the variations of SEPs on long-term scales.

It is not straightforward to evaluate the average SEP flux even for the modern instrumental epoch of direct space-borne measurements (e.g., Mewaldt et al. 2007). For example, estimates for the average flux of SEPs with an energy above $30 \mathrm{MeV}$ (called $F_{30}$ henceforth) for individual cycles may vary by an order of magnitude, from

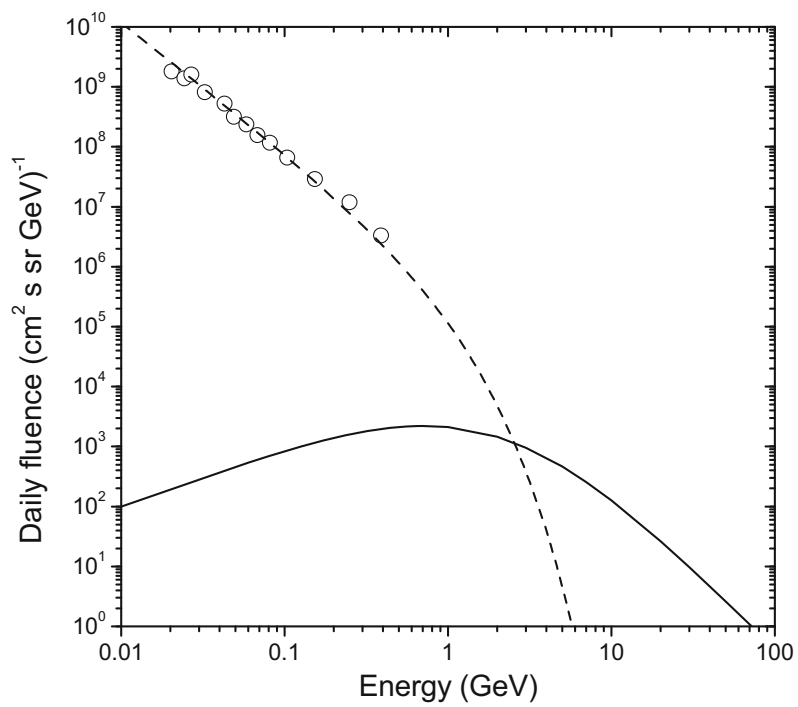

Fig. 23 Daily fluence of solar energetic particles (dashed curve-Tylka and Dietrich 2009) and galactic cosmic rays (solid curve) for the day of January 20, 2005. Open circles represent space-borne measurements (Mewaldt 2006; Mewaldt et al. 2012) 
$10 \mathrm{~cm}^{-2} \mathrm{~s}^{-1}$ for cycle 21 up to $70 \mathrm{~cm}^{-2} \mathrm{~s}^{-1}$ for cycle 19 (Reedy 2012). Moreover, estimates of the SEP flux were quite uncertain during the earlier years of space-borne measurements because of two effects, which are hard to account for (e.g., Reeves et al. 1992; Tylka et al. 1997). One is related to the very high flux intensities of SEPs during the peak phase of events, when a detector can be saturated because of the dead-time effect (the maximum trigger rate of the detector is exceeded). The other is related to events with high energy solar particles, which can penetrate into the detector through the walls of the collimator, leading to an enhanced effective acceptance cone with respect to the "expected" one. Since the SEP fluence is defined by major events, these effects may lead to an underestimate of the average flux of SEPs. The modern generation of detectors are better suited for measuring high fluxes. The average $F_{30}$ flux for the last five solar cycles (1954-2006) is estimated at about $35 \mathrm{~cm}^{-2} \mathrm{~s}^{-1}$ (Smart and Shea 2002; Shea et al. 2006).

\subsection{Cosmogenic isotopes}

The development of the method of cosmogenic isotopes makes it possible to estimate occurrence of extreme SEP events in the past. Some earlier attempts were inconclusive. For example, Usoskin et al. (2006b) found that a typical strong SEP event leaves no distinguishable signature in ${ }^{14} \mathrm{C}$ but may be observed from ice core ${ }^{10} \mathrm{Be}$ records. However, the question of the possible rare occurrence of extreme SEP events on the millennial time scale is important not only from the theoretical point of view, but also for assessment of radiation risks for space-borne missions, especially manned ones. What can be the strongest SEP event originated from the sun, how often they can occur? These questions need to be answered. Several attempts have been made to evaluate that from the cosmogenic isotope data (Lingenfelter and Hudson 1980; Usoskin et al. 2006b; Webber et al. 2007), but the result was grossly uncertain (Hudson 2010; Schrijver et al. 2012), mostly because of the large model uncertainties of the radionuclide production.

A new step forward has been done recently by Usoskin and Kovaltsov (2012), who analyzed two ${ }^{14} \mathrm{C}$ and five ${ }^{10} \mathrm{Be}$ records over the last millennia and searched for possible signatures of extreme SEP events.

While the response of ${ }^{10} \mathrm{Be}$ to an SEP event is simply a 1-2-years long peak, because of the simple atmospheric transport/deposition (see Sect. 3.3.3), the response of ${ }^{14} \mathrm{C}$ has a typical shape shown in Fig. 24-with a sharp peak and exponential decay of the length of several decades, due to the carbon cycle (see Sect. 3.2.3). A search for the available cosmogenic isotope data has been performed through millennia (Usoskin and Kovaltsov 2012; Miyake et al. 2016), looking for a potential SEP signatures, and came up with a list of candidates of extreme SEP events and assessments of their strength (Table 4).

The list includes 23 candidates for extreme SEP events with the fluence $F_{30}$ exceeding $10^{10} \mathrm{~cm}^{-2}$, viz. the greatest fluence observed for the space era in 1960 (Shea and Smart 1990). Note that only two of these candidates appear in more than one seriesthe events of ca. $1460 \mathrm{AD}$ and ca. $780 \mathrm{AD}$. The former had signatures in two annual ${ }^{10} \mathrm{Be}$ series, NGRIP and Dye 3. The later was observed in two ${ }^{14} \mathrm{C}$ series, biennial M12 


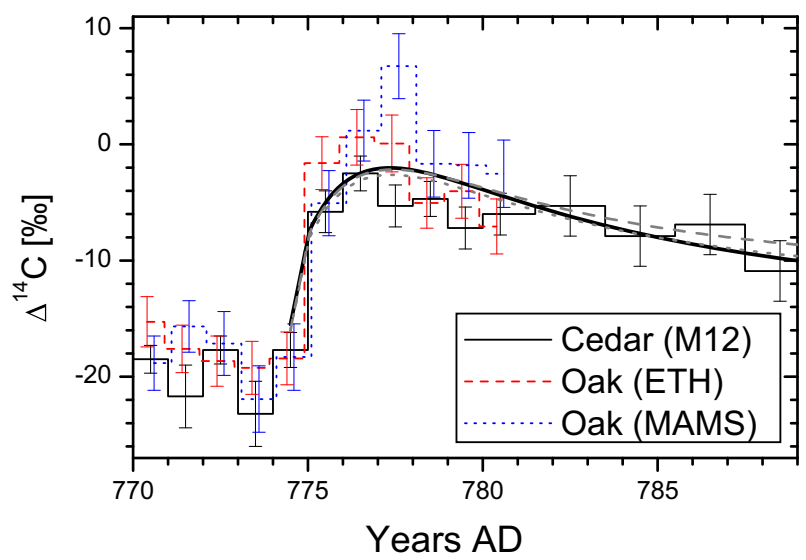

Fig. 24 Time profiles of the measured $\Delta^{14} \mathrm{C}$ content in Japanese cedar (M12-Miyake et al. 2012) and German oak (ETH Zürich \& Mannheim AMS_-Usoskin et al. 2013) trees for the period around 775 AD. Smooth black and grey lines depict a family of best fit $\Delta^{14} \mathrm{C}$ profiles, calculated using a family of realistic carbon cycle models for an instantaneous injection of ${ }^{14} \mathrm{C}$ into the stratosphere (Usoskin and Kovaltsov 2012). Image after Usoskin et al. (2013)

and 5-years IntCal13, and in quasi-decadal Dome Fuji ${ }^{10} \mathrm{Be}$ series. The quasi-decadal South Pole ${ }^{10} \mathrm{Be}$ series does not show an increase ca. $780 \mathrm{AD}$ placing an upper limit on the strength of the event.

\subsubsection{The event of 775 AD: the worst case scenario?}

The event of ca. $775 \mathrm{AD}$ was analyzed using biennial ${ }^{14} \mathrm{C}$ data by Miyake et al. (2012), who suggested that the event was probably caused by $\gamma$-rays from an unknown nearby supernova. This event is confirmed by annual ${ }^{14} \mathrm{C}$ data from a German oak tree (Usoskin et al. 2013), Russian and American tree samples (Jull et al. 2014), New Zealand trees (Güttler et al. 2013), etc., and corals from the Chinese Sea (Liu et al. 2014) According to model simulations, the production of ${ }^{14} \mathrm{C}$ appears in agreement with that of ${ }^{10} \mathrm{Be}$ (Usoskin et al. 2013; Melott and Thomas 2012; Pavlov et al. 2013). Although some exotic scenarios were proposed for the event: an unidentified nearby supernova (Miyake et al. 2012); a gamma-ray burst (Hambaryan and Neuhäuser 2013; Pavlov et al. 2013); or even a cometary impact on Earth (Liu et al. 2014), it is generally accepted now that it was a signature of a (probably, consequence of) extreme SEP event (Usoskin and Kovaltsov 2012; Eichler and Mordecai 2012; Usoskin et al. 2013; Melott and Thomas 2012; Thomas et al. 2013; Cliver et al. 2014). A detailed analysis performed by Mekhaldi et al. (2015) not only confirmed its solar origin but also made it possible to assess, based on data from different cosmogenic isotopes, the reconstructed integral spectrum as shown in Fig. 25 along with the fluence spectra of two extreme SEP events of the space era: the hardest event of 23-Feb-1956 (GLE \# 5) with the greatest fluence of high energy SEPs; and the event of 04-Aug-1972 with the greatest measured fluence of lower energy SEPs. One can see that the energy spectrum of the 775 AD event was very hard, close to that of the 23-Feb-1956 event, but scaled up by 
Table 4 A list of candidates for extreme SEP events found in different cosmogenic isotope records throughout the

Holocene: approximate year, dataset used

(Dye3-McCracken et al.

(2004); NGRIP_-Berggren et al. (2009); IntCal09_-Reimer et al. (2009); GRIP_Yiou et al.

(1997); Dome Fuji-Horiuchi et al. (2008); South

Pole-Raisbeck et al. (1990); M12-Miyake et al. (2012)), and the $F_{30}$ fluence $\left(\mathrm{cm}^{-2}\right)$

Table after Usoskin and Kovaltsov (2012)

a Upper bound

\begin{tabular}{|c|c|c|}
\hline SPE year & Series & $F_{30}$ \\
\hline \multirow[t]{2}{*}{$1460-1462$ AD } & NGRIP(1460) & $1.5 \times 10^{10}$ \\
\hline & Dye3 (1462) & $9.7 \times 10^{9}$ \\
\hline $1505 \mathrm{AD}$ & Dye3 & $1.3 \times 10^{10}$ \\
\hline 1719 AD & NGRIP & $1 \times 10^{10}$ \\
\hline $1810 \mathrm{AD}$ & NGRIP & $1 \times 10^{10}$ \\
\hline 8910 BC & IntCa109 & $2.0 \times 10^{10}$ \\
\hline 8155 BC & IntCal09 & $1.3 \times 10^{10}$ \\
\hline 8085 BC & IntCal09 & $1.5 \times 10^{10}$ \\
\hline 7930 BC & IntCal09 & $1.3 \times 10^{10}$ \\
\hline 7570 BC & IntCal09 & $2.0 \times 10^{10}$ \\
\hline 7455 BC & IntCa109 & $1.5 \times 10^{10}$ \\
\hline 6940 BC & IntCal09 & $1.1 \times 10^{10}$ \\
\hline $6585 \mathrm{BC}$ & IntCal09 & $1.7 \times 10^{10}$ \\
\hline 5835 BC & IntCal09 & $1.5 \times 10^{10}$ \\
\hline 5165 BC & GRIP & $2.4 \times 10^{10}$ \\
\hline $4680 \mathrm{BC}$ & IntCal09 & $1.6 \times 10^{10}$ \\
\hline 3260 BC & IntCal09 & $2.4 \times 10^{10}$ \\
\hline 2615 BC & IntCal09 & $1.2 \times 10^{10}$ \\
\hline 2225 BC & IntCal09 & $1.2 \times 10^{10}$ \\
\hline 1485 BC & IntCal09 & $2.0 \times 10^{10}$ \\
\hline $95 \mathrm{AD}$ & GRIP & $2.6 \times 10^{10}$ \\
\hline $265 \mathrm{AD}$ & IntCa109 & $2.0 \times 10^{10}$ \\
\hline \multirow[t]{3}{*}{$785 \mathrm{AD}$} & IntCal09 & $2.4 \times 10^{10}$ \\
\hline & Dome Fuji & $5.3 \times 10^{10 a}$ \\
\hline & M12 & $4 \times 10^{10 a}$ \\
\hline $1455 \mathrm{AD}$ & South Pole & $7.0 \times 10^{10 a}$ \\
\hline
\end{tabular}

a factor $\approx 40$. This implies that the fluence of high energy particles was much greater (factor of $\approx 40$ ) in $775 \mathrm{AD}$ than ever observed during the space era. On the other hand, the low-energy fluence (energy range of several tens of $\mathrm{MeV}$ ) for the $775 \mathrm{AD}$ event was only a factor of two greater than that of the event of Aug-1972, suggesting that the fluence of low-energy SEP might have a natural limit (Asvestari et al. 2016), for example related to the "streaming limit" (Reames and Ng 2010). Thus, the high fluence of high energy particles does not necessarily mean a very high fluence of the lower-energy particles.

The signal of the $775 \mathrm{AD}$ event was so strong and clearly visible in the ice-core ${ }^{10} \mathrm{Be}$ data that it is now used as a tie point (the point with independently known date, e.g., volcanic eruption or, as in this case, the SEP event) for more precise dating of ice cores (Sigl et al. 2015). 


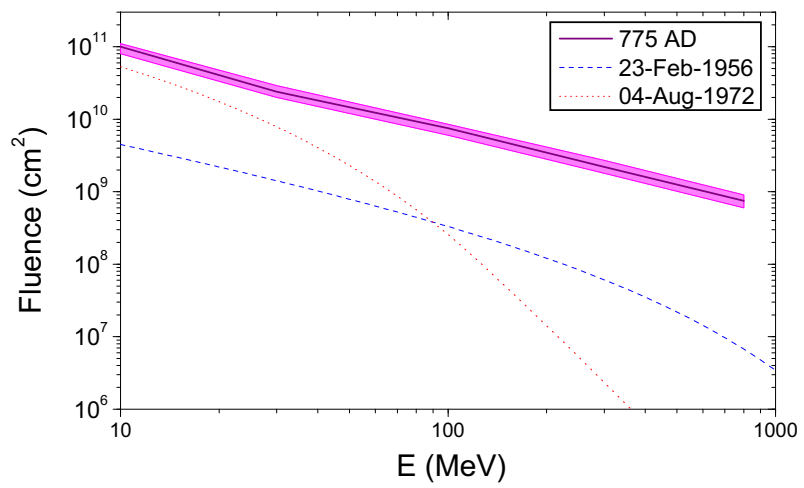

Fig. 25 Even-integrated SEP fluence spectra for the 775 AD event (Mekhaldi et al. 2015), the hardest known and the greatest soft-spectrum SEP events of 23-Feb-1956 and 04-Aug-1972, respectively (Tylka and Dietrich 2009)

Another similar event was found to occur in 994 AD (Miyake et al. 2013), that was roughly half of that of $775 \mathrm{AD}$ and also had a very hard spectrum (Mekhaldi et al. 2015).

Do we expect that even stronger SEP events took place in the past? The $775 \mathrm{AD}$ event was observed as the sharpest peak $(\approx 0.4 \%$ years $)$ in the decadal ${ }^{14} \mathrm{C}$ IntCal dataset, while the smaller peak $(\approx 0.35 \% /$ years $)$ of the 994 AD was only barely seen in the IntCal data. Recently, Miyake et al. (2016) measured, with (bi)annual resolution, ${ }^{14} \mathrm{C}$ around four smaller peaks $(\approx 0.3 \%$ /years) since $4800 \mathrm{BC}$ and found no other events, suggesting for uniqueness of the $775 \mathrm{AD}$ one for the last six millennia. We note that other remaining peaks in the IntCal data are smaller than the one of 775 AD. Accordingly, an event stronger than that of 775 AD could be found only in a case of an unlikely random coincidence of the event itself with an incidental drop of ${ }^{14} \mathrm{C}$ caused by other reasons, masking the spike. In particular, the event twice as strong as the $775 \mathrm{AD}$ one is hardly possible to occur since it would have produced a large spike in the IntCal data which could not be missed (see Fig. 26). Thus, the 775 AD event can securely serve as the worst case scenario of the SEP event during the entire Holocene.

\subsubsection{Occurrence rate}

The integral probability distribution of the occurrence of strong SEP events, as revealed from the cosmogenic isotope data, is shown in Fig. 27.

One can see that the break in the distribution marginally hinted in the directly observed SEP events at around $F_{30}=(5-7) \times 10^{9} \mathrm{~cm}^{-2}$ (nonproportionally fewer strong events observed) is confidently confirmed by the cosmogenic isotope data. In particular, no event with $F_{30}>2 \times 10^{10} \mathrm{~cm}^{-2}$ was found over the last 600 years using annually resolved ${ }^{10} \mathrm{Be}$ data. It is noteworthy that the idea of a possible extreme Carrington SPE of 1859 AD (McCracken et al. 2001) is discarded (see also Wolff et al. 2012). On the longer time scale of 11 millennia, no event with $F_{30}>5 \times 10^{10} \mathrm{~cm}^{-2}$ 


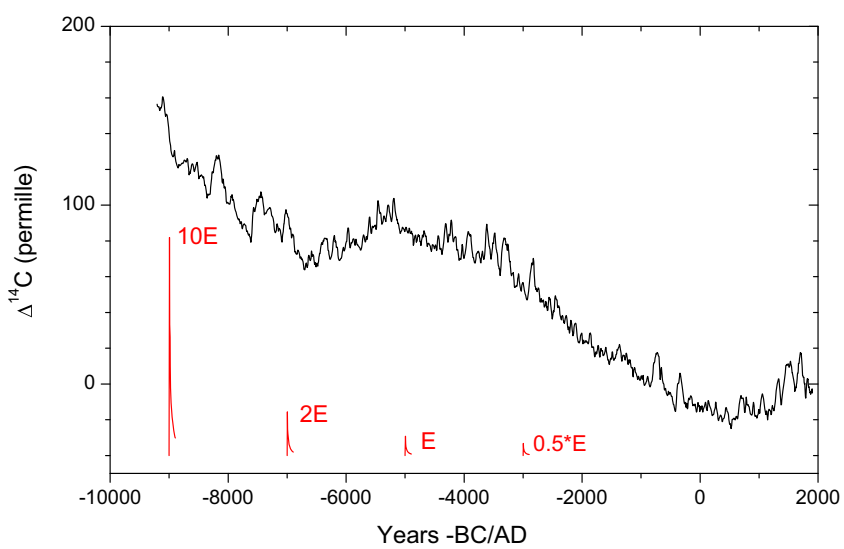

Fig. 26 The record (black) of $\Delta^{14} \mathrm{C}$ (IntCal09 Reimer et al. 2009) throughout the Holocene, along with the expected signal (red) in the decadal $\Delta^{14} \mathrm{C}$ data, from a scaled $775 \mathrm{AD}$ event (denoted as " $\mathrm{E}$ ")

Fig. 27 Cumulative probability (with the $90 \%$ confidence interval) of occurrence of a SEP event with fluence $(>30 \mathrm{MeV})$ exceeding the given value $F_{30}$, as assessed from the data for the space era 1956-2008 (black triangles), cosmogenic isotope data (blue circles), and cosmogenic data from lunar rocks (red shaded area). Modified after Kovaltsov and Usoskin (2014)

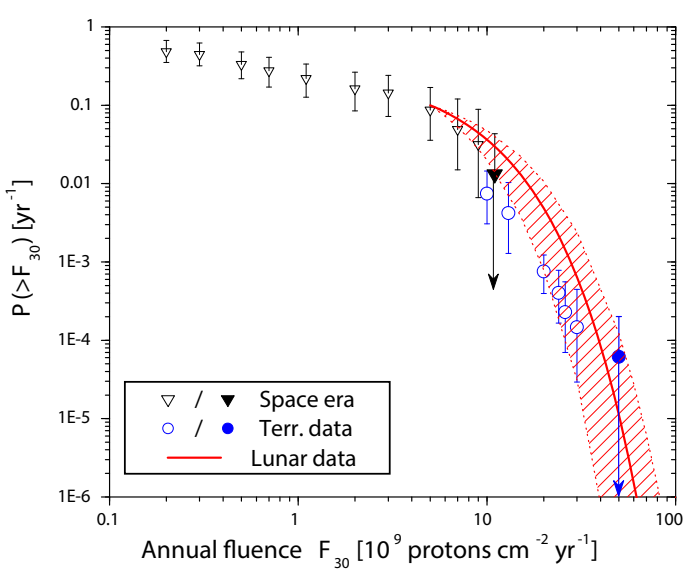

has been found. This gives a new strict observational constraint on the occurrence probability of extreme SPEs.

According to Usoskin and Kovaltsov (2012) practical limits can be set as $F_{30} \approx 1$, 2-3 and $5 \times 10^{10} \mathrm{~cm}^{-2}(10,20-30$ and 50 times greater than the SEP event of February 23,1956$)$, for the occurrence probability of $10^{-2}, 10^{-3}$, and $10^{-4}$ years $^{-1}$, respectively. The mean SEP flux is found as $\approx 40\left(\mathrm{~cm}^{2} \mathrm{~s}\right)^{-1}$ in agreement with estimates from the lunar rocks. On average, extreme SPEs contribute about $10 \%$ to the total SEP fluence.

\subsection{Lunar and meteoritic rocks}

Since energy spectra of SEP and GCR are dramatically different, one may think of a natural spectrometer to separate their effects and thus evaluate their fluxes indepen- 


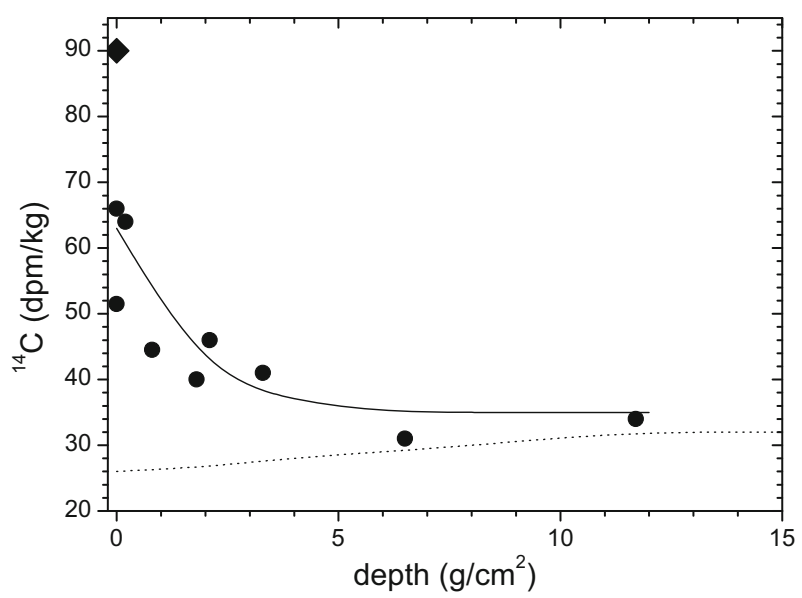

Fig. 28 Measured (dots) and calculated (curves) ${ }^{14} \mathrm{C}$ activity in a lunar sample 68815 (Jull et al. 1998). The big diamond implies contamination of a thin surface layer by ${ }^{14} \mathrm{C}$ implanted from solar wind. The dotted curve represents the expected production due to GCR, while the solid curve is the best fit SEP+GCR model production

dently. A spectrometer that is able to separate cosmic rays is lunar (or meteoritic) rocks.

Figure 28 depicts an example of ${ }^{14} \mathrm{C}$ measured in a lunar sample (Jull et al. 1998). The dotted line shows the expected production of radiocarbon by GCR. The production increases with depth due to the development of a nucleonic cascade in the matter, initiated by energetic GCR particles, similar to the atmospheric cascade. Less energetic particles of solar origin produce the isotope only in upper layers of the rock, since their low energy does not allow them to initiate a cascade. On the other hand, thanks to their high flux in the lower energy range, the production of ${ }^{14} \mathrm{C}$ in the upper layers is much higher than that from GCR. Thus, by first measuring the isotope activity in deep layers one can evaluate the average GCR flux, and then the measured excess in the upper level yields an estimate for the SEP flux in both integral intensity and spectral shape. The result is based on model computations and therefore is slightly model dependent but makes it possible to give a robust estimate of the GCR and SEP in the past.

A disadvantage of this approach is that lunar samples are not stratified and do not allow for temporal separation. The measured isotope activity is a balance between production and decay and, therefore, represents the production (and the ensuing flux) integrated over the life-time of the isotope before the sample has been measured. However, using different isotopes with different life times, one can evaluate the cosmicray flux integrated over different timescales.

Estimates of the average SEP flux $F_{30}$ on different timescales, as obtained from various isotopes measured in lunar samples, are collected in Table 5. Based on isotopes with different life-times (see Table 5) one can evaluate the average flux of SEP on different time scale (see Fig. 29). The average $F_{30}$ flux for the last five solar cycles (1954-2008) is consistent with the average flux estimated in the past for longer timescales from $10^{3}$ to $10^{7}$ years (cf. Reedy 2002, 2012). 
Table 5 Estimates of $4 \pi$ omni-directional integral (above $30 \mathrm{MeV}$ ) flux, $F_{30}$ in $\left(\mathrm{cm}^{2} \mathrm{~s}\right)^{-1}$, of solar energetic particles, obtained from different sources

\begin{tabular}{lllll}
\hline Timescale & Method & Source & References & $F_{30}\left(\mathrm{~cm}^{-2} \mathrm{~s}^{-1}\right)$ \\
\hline $1954-2008$ & Measurements & Space-borne & Reedy (2012) & 35 \\
$10^{4}$ years & ${ }^{14} \mathrm{C}$ & Lunar rock & Jull et al. (1998) & 42 \\
$10^{5}$ years & ${ }^{41} \mathrm{Ca}$ & Lunar rock & Fink et al. (1998) & 56 \\
$5 \times 10^{5}$ years & ${ }^{36} \mathrm{Cl}$ & Lunar rock & Nishiizumi et al. (2009) & 46 \\
$10^{6}$ years & ${ }^{26} \mathrm{Al}$ & Lunar rock & Kohl et al. (1978) & 25 \\
$10^{6}$ years & ${ }^{26} \mathrm{Al}$ & Lunar rock & Grismore et al. (2001) & 55 \\
$10^{6}$ years & ${ }^{10} \mathrm{Be},{ }^{26} \mathrm{Al}$ & Lunar rock & Michel et al. (1996) & 24 \\
$10^{6}$ years & ${ }^{10} \mathrm{Be},{ }^{26} \mathrm{Al}$ & Lunar rock & Fink et al. (1998) & 32 \\
$10^{6}$ years & ${ }^{10} \mathrm{Be},{ }^{26} \mathrm{Al}$ & Lunar rock & Nishiizumi et al. (2009) & 24 \\
$2 \times 10^{6}$ years & ${ }^{10} \mathrm{Be},{ }^{26} \mathrm{Al}$ & Lunar rock & Nishizumi et al. (1997) & $\sim 35$ \\
$5 \times 10^{6}$ years & ${ }^{53} \mathrm{Mn}$ & Lunar rock & Kohl et al. (1978) & 25 \\
$2 \times 10^{6}$ years & ${ }^{21} \mathrm{Ne},{ }^{22} \mathrm{Ne},{ }^{38} \mathrm{Ar}$ & Lunar rock & Rao et al. (1994) & 22 \\
\hline
\end{tabular}

However, this method is not able to provide an estimate of the occurrence rate of extreme SEP events. If one assumes that the entire average SEP flux is produced within one extreme event occurring at half of the isotope's life-time ago (Reedy 1996), an upper limit for the occurrence of extreme SEP events can be placed. This is an unrealistically extreme assumption, which may lead to an overestimate by many orders of magnitude, but it sets the very conservative upper limit which cannot be exceeded.

A more realistic assumption is based on a distribution of SEP events in strength, for example exponential. If applying this, the distribution of the SEP events estimated from lunar rocks appears fully consistent with the assessments based on terrestrial cosmogenic isotope data and direct data on SEP fluences for the space era (Fig. 27).

\subsection{Nitrates in polar ice}

It has been discussed until recently that another quantitative index of strong SEP events (with $F_{30}>10^{9} \mathrm{~cm}^{-2}$ ) might be related to nitrate $\left(\mathrm{NO}_{3}^{-}\right)$records measured in polar ice cores. The concentration of nitrates has been measured in polar ice from both the Southern (South Pole, e.g., Dreschhoff and Zeller 1990) and Northern (Greenland, e.g., Zeller and Dreschhoff 1995; Dreschhoff and Zeller 1998) polar caps, depicting pronounced spikes associated with strong SEP events (McCracken et al. 2001). As a result of the analysis a list of large SEP events since 1560 and their fluences have been published (see Table 1 in McCracken et al. 2001) and widely used.

However, as shown by several independent recent studies (Wolff et al. 2012; Usoskin and Kovaltsov 2012) on the example of the Carrington event (September 1859), the nitrate spikes are not related to SEP events. According to McCracken et al. (2001), the nitrate spike and the associated SEP event was the strongest in the entire record $\left(F_{30} \approx 2 \times 10^{10} \mathrm{~cm}^{-2}\right)$. Wolff et al. (2012) have measured, with high resolution, nitrate 
Fig. 29 Cumulative frequency distribution of SEP events with fluences greater than $F_{10}$ (for particles with energies above $10 \mathrm{MeV})$. Red histogram: satellite-based direct observations; Blue diamonds: conservative upper limits derived from lunar isotopes (see Sect. 5.2); Blue dashed line: upper limit based on ${ }^{14} \mathrm{C}$ record (Hudson 2010); Image reproduced by permission from Schrijver et al. (2012), copyright by AGU

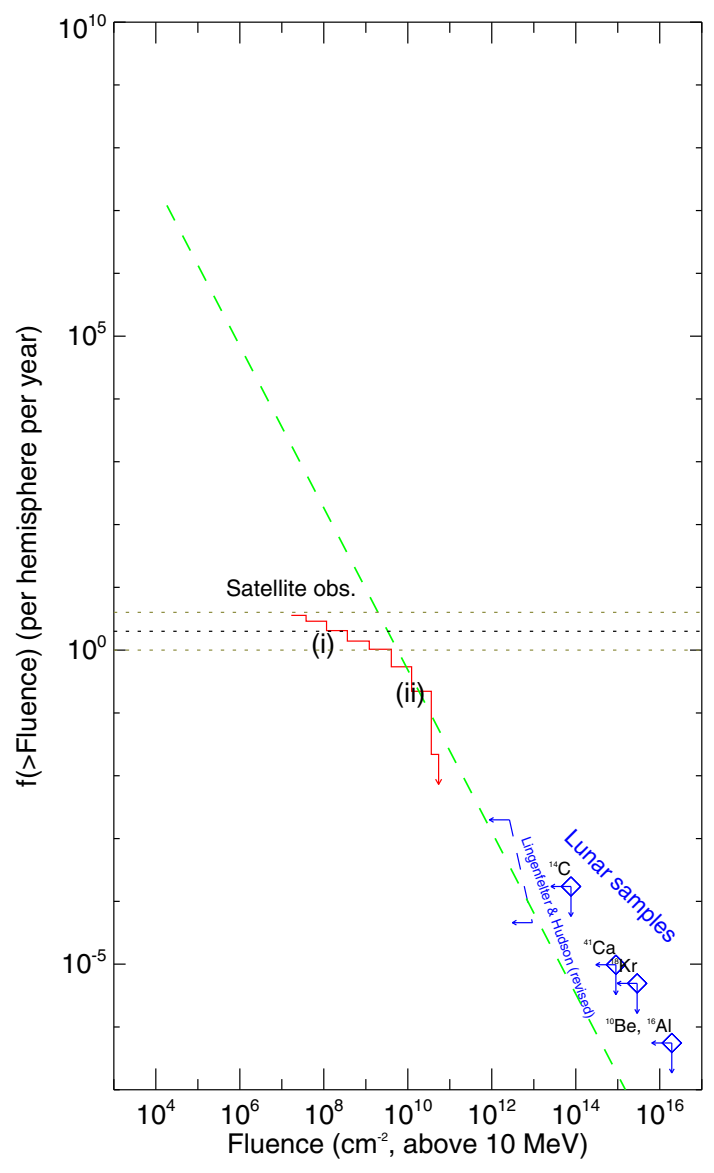

content in 14 ice cores from Antarctic and Greenland for a few decades around 1859. Only one Greenland series depicts a spike which can be associated with the event, all other series have no signatures. Moreover, all similar spikes found in Greenland datasets are accompanied by chemical tracers (ammonium, formate, black carbon, etc.) clearly pointing to the anthropogenic source of nitrates-biomass burning plumes. No significant spikes have been found in the Antarctic records. Wolff et al. (2012) concluded that "Nitrate spikes cannot be used to derive the statistics of SEPs".

This work was criticized by Smart et al. (2014), who stated that the resolution of the analyzed series was not high enough and could have led to missing the spike, which was counter-argued by Wolff et al. (2016) that even a fine time resolution does not reveal the peaks. It was also discussed theoretically (Duderstadt et al. 2016) that a realistic SEP event can hardly produce sufficient amount of nitrate to leave a strong pulse-like signature in an ice core. Usoskin and Kovaltsov (2012) calculated, from the $F_{30}$ fluence proposed by McCracken et al. (2001) for the Carrington event, the ${ }^{10} \mathrm{Be}$ production and showed that, if the Carrington SEP event was so strong, it would 
have necessarily produce a spike in the annually resolved ${ }^{10} \mathrm{Be}$ record, which however contradicts to the real data from NGRIP and Dye 3 ice cores.

Thus, the nitrate record in polar ice cannot serve as an index of SEP events. On the other hand, it may be used to study long-term variability of GCR (see Sect. 3.4).

\subsection{Summary}

In this section, estimates of the averaged long-term flux of SEPs are discussed.

Measurements of cosmogenic isotopes with different life times in lunar and meteoritic rocks allow one to make rough estimates of the SEP flux over different timescales. The directly space-borne-measured SEP flux for past decades is broadly consistent with estimates on longer timescales - up to millions of years. The same measurements can provide a very conservative upper estimate for the occurrence rate of extreme SEP events. Terrestrial cosmogenic isotope data in dated archives (tree trunks, ice cores) give a possibility to assess the occurrence rate of strong SEP events on the time scales up to ten of millennia. Measurements of nitrates in polar ice have been shown to be an invalid index of strong SEP events in the past.

The strongest known SEP event has occurred in $775 \mathrm{AD}$ as found in terrestrial cosmogenic isotope data. It can server as the worst case scenario for an extreme SEP event on the multi-millennial time scale. A smaller, but still extreme, event took place in 994 AD.

Different estimates of the extreme (quantified as the fluence of SEP with energy above $10 \mathrm{MeV}$ ) SEP event occurrence probability are summarized in Fig. 29.

An analysis of various kinds of data suggests that the distribution of the intensity of SEP events has a break, and the occurrence of extra-strong events (with the $F_{30}$ fluence exceeding $5 \times 10^{10} \mathrm{~cm}^{-2}$ ) is unlikely on the multi-millennial time scale.

\section{Conclusions}

In this review the present knowledge of long-term solar activity on a multi-millennial timescale, as reconstructed using the indirect proxy method, is discussed.

Although the concept of solar activity is intuitively understandable as a deviation from the "quiet" sun concept, there is no clear definition for it, and different indices have been proposed to quantify different aspects of variable solar activity. One of the most common and practical indices is sunspot number, which forms the longest available series of direct scientific observations. While all other indices have a high correlation with sunspot numbers, dominated by the 11-year cycle, the relationship between them at other timescales (short- and long-term trends) may vary to a great extent.

On longer timescales, quantitative information of past solar activity can only be obtained using the method based upon indirect proxy, i.e., quantitative parameters, which can be measured nowadays but represent the signatures, stored in natural archives, of the different effects of solar magnetic activity in the past. Such traceable signatures can be related to nuclear or chemical effects caused by cosmic rays in the Earth's atmosphere, lunar rocks or meteorites. The most common proxy of 
solar activity is formed by data from the cosmogenic radionuclides, ${ }^{10} \mathrm{Be}$ and ${ }^{14} \mathrm{C}$, produced by cosmic rays in the Earth's atmosphere and stored in independently-dated stratified natural archives, such as tree rings or ice cores. Using a recently-developed physics-based model it is now possible to reconstruct the temporal behavior of solar activity in the past, over many millennia. The most robust results can be obtained for the Holocene epoch, which started more than 11000 years ago, whose stable climate minimizes possible uncertainties in the reconstruction. An indirect verification of long-term solar-activity reconstructions supports their veracity and confirms that variations of cosmogenic nuclides on the long-term scale (centuries to millennia) during the Holocene make a solid basis for studies of solar variability in the past. However, such reconstructions may still contain systematic uncertainties related to unknown changes in the geomagnetic field or climate of the past, especially in the early part of the Holocene.

Measurements of the concentration of different cosmogenic isotopes in lunar and meteoritic rocks make it possible to estimate the SEP flux on different timescales. Directly space-borne-measured SEP flux for recent decades is broadly consistent with estimates on longer timescales - up to millions of years. The occurrence of extrastrong events, with the fluence of SEP (with energy greater than $30 \mathrm{MeV}$ ) exceeding $5 \times 10^{10} \mathrm{~cm}^{-2}$ is unlikely on the multi-millennial time scale. The SEP event of $775 \mathrm{AD}$, discovered using data of cosmogenic isotopes, was the strongest known events, which can serve as the worst case scenario for the entire Holocene.

In general, the following main features are observed in the long-term evolution of solar magnetic activity.

- Solar activity is dominated by the 11-year Schwabe cycle on an interannual timescale. Some additional longer characteristic times can be found, including the Gleissberg secular cycle, de Vries/Suess cycle, and a quasi-cycle of 20002400 years (Hallstatt cycle). However, all these longer cycles are intermittent and cannot be regarded as strict phase-locked periodicities.

- One of the main features of long-term solar activity is that it contains an essential chaotic/ stochastic component, which leads to irregular variations and makes solaractivity predictions impossible for a scale exceeding one solar cycle.

- The sun spends about $70 \%$ of its time at moderate magnetic activity levels, about $15-20 \%$ of its time in a grand minimum and about $10-15 \%$ in a grand maximum.

- Grand minima are a typical but rare phenomena in solar behavior. They form a distinct mode of solar dynamo. Their occurrence appears not periodically, but rather as the result of a chaotic process within clusters separated by the 20002500 years (around the lows of the Hallstatt cycle). Grand minima tend to be of two distinct types: short (Maunder-like) and longer (Spörer-like).

- The recent level of solar activity (after the 1940s) was very high, corresponding to a prolonged grand maximum, but it has ceased to the normal moderate level. Grand maxima are also rare and irregularly occurring events, though the exact rate of their occurrence is still a subject of debates.

These observational features of the long-term behavior of solar activity have important implications, especially for the development of theoretical solar-dynamo models and for solar-terrestrial studies. 
Acknowledgements I thank the editorial board of the Living Reviews in Solar Physics for the invitation to prepare this review and organizational aid. This work would be impossible without encouraging discussions, as well as direct and indirect help, from my colleagues Gennady Kovaltsov, Kalevi Mursula and Sami Solanki, whose invaluable support is acknowledged with my greatest gratitude. I have also greatly benefited from lively and open discussions and debates with Rainer Arlt, Edouard Bard, Jürg Beer, Narendra Bhandari, late Giuliana Cini Castagnoli, Paul Charbonneau, Ed Cliver, Vincent Courtillot, Cornelis de Jager, Valentin Dergachev, Gisella Dreschhoff, Erwin Flückiger, Peter Foukal, Yves Gallet, Agnés Genevey, David Hathaway, Bernd Heber, Fadil Inceoglu, Mads Knudsen, Monika Korte, Natalia Krivova, Bernd Kromer, Maarit Käpylä, Devendra Lal, Michael Lockwood, Ken McCracken, David Moss, late Harm Moraal, Kalevi Mursula, Raimund Muscheler, Hiroko Miyahara, Heikki Nevanlinna, Keran O’Brien, Valery Ostryakov, Anatoly Pavlov, Alexander Ruzmaikin, late Dieter Schmitt, Manfred Schüssler, Margaret Shea, Don Smart, Ian Snowball, Dmitry Sokoloff, Willie Soon, Leif Svalgaard, Carla Taricco, Rita Traversi, Jóse Vaquero, and many others. I am happy to acknowledge those colleagues who work in this field and make it living and vibrant. This work was supported by the Center of Excellence ReSoLVE (Project 272157) of the Academy of Finland.

Open Access This article is distributed under the terms of the Creative Commons Attribution 4.0 International License (http://creativecommons.org/licenses/by/4.0/), which permits unrestricted use, distribution, and reproduction in any medium, provided you give appropriate credit to the original author(s) and the source, provide a link to the Creative Commons license, and indicate if changes were made.

\section{References}

Abreu JA, Beer J, Steinhilber F, Tobias SM, Weiss NO (2008) For how long will the current grand maximum of solar activity persist? Geophys Res Lett 35:L20109. doi:10.1029/2008GL035442

Abreu JA, Beer J, Ferriz-Mas A, McCracken KG, Steinhilber F (2012) Is there a planetary influence on solar activity? Astron Astrophys 548:A88. doi:10.1051/0004-6361/201219997

Adolphi F, Muscheler R (2016) Synchronizing the Greenland ice core and radiocarbon timescales over the Holocene-Bayesian wiggle-matching of cosmogenic radionuclide records. Clim Past 12:15-30. doi:10.5194/cp-12-15-2016

Aparicio AJP, Vaquero JM, Carrasco VMS, Gallego MC (2014) Sunspot numbers and areas from the madrid astronomical observatory (1876-1986). Solar Phys 289:4335-4349. doi:10.1007/s11207-014-0567-x. arXiv: 1407.3104

Argento DC, Reedy RC, Stone JO (2013) Modeling the Earth's cosmic radiation. Nucl Instrum Methods B 294:464-469. doi:10.1016/j.nimb.2012.05.022

Arlt R (2008) Digitization of sunspot drawings by Staudacher in 1749-1796. Solar Phys 247:399-410. doi:10.1007/s11207-007-9113-4

Arlt R (2009) The butterfly diagram in the eighteenth century. Solar Phys 255:143-153. doi:10.1007/ s11207-008-9306-5. arXiv:0812.2233

Arlt R, Abdolvand A (2011) First solar butterfly diagram from Schwabe's observations in 1825-1867. In: Choudhary DP, Strassmeier KG (eds) Physics of sun and star spots, IAU symposium, vol S273. Cambridge University Press, Cambridge, pp 286-289. doi:10.1017/S1743921311015390. arxiv.org/abs/1010.3131

Arlt R, Leussu R, Giese N, Mursula K, Usoskin I (2013) Sunspot positions and sizes for 1825-1867 from the observations by Samuel Heinrich Schwabe. Mon Not R Astron Soc 433:3165-3172. doi:10.1093/ mnras/stt961. arXiv:1305.7400

Asvestari E, Willamo T, Gil A, Usoskin I, Kovaltsov G, Mikhailov V, Mayorov A (2016) Analysis of ground level enhancements (GLE): extreme solar energetic particle events have hard spectra. Adv Space Res (in press), doi:10.1016/j.asr.2016.08.043

Asvestari E, Usoskin IG, Kovaltsov GA, Owens MJ, Krivova NA, Rubinetti S, Taricco C (2017) Assessment of different sunspot number series using the cosmogenic isotope 44Ti in meteorites. Mon Not Royal Astron Soc 467:1608-1613. doi:10.1093/mnras/stx190

Aulanier G, Démoulin P, Schrijver CJ, Janvier M, Pariat E, Schmieder B (2013) The standard flare model in three dimensions. II. Upper limit on solar flare energy. Astron Astrophys 549:A66. doi:10.1051/ 0004-6361/201220406. arXiv:1212.2086 
Balmaceda LA, Solanki SK, Krivova NA (2005) A cross-calibrated sunspot areas time series since 1874. Mem Soc Astron Ital 76:929-932

Baranyi T, Gyõri L, Ludmány A, Coffey HE (2001) Comparison of sunspot area data bases. Mon Not R Astron Soc 323:223-230. doi:10.1046/j.1365-8711.2001.04195.x

Bard E, Arnold M, Maurice P, Duplessy JC (1987) Measurements of bomb radiocarbon in the ocean by means of accelerator mass spectrometry: Technical aspects. Nucl Instrum Methods B 29:297-301. doi:10.1016/0168-583X(87)90253-9

Bard E, Raisbeck GM, Yiou F, Jouzel J (1997) Solar modulation of cosmogenic nuclide production over the last millenium: comparison between ${ }^{14} \mathrm{C}$ and ${ }^{10} \mathrm{Be}$ records. Earth Planet Sci Lett 150:453-462. doi:10.1016/S0012-821X(97)00082-4

Barnard L, Lockwood M, Hapgood MA, Owens MJ, Davis CJ, Steinhilber F (2011) Predicting space climate change. Geophys Res Lett 38:L16103. doi:10.1029/2011GL048489

Basurah HM (2004) Auroral evidence for early high solar activities. Solar Phys 225:209-212. doi:10.1007/ s11207-004-1372-8

Beer J (2000) Long-term indirect indices of solar variability. Space Sci Rev 94(1/2):53-66. doi:10.1023/A: 1026778013901

Beer J, Siegenthaler U, Oeschger H, Andrée M, Bonani G, Suter M, Wolfli W, Finkel RC, Langway CC (1983) Temporal ${ }^{10} \mathrm{Be}$ variations. In: Durgaprasad D (ed) 18th International cosmic ray conference, vol 9. TIFR, Bombay, pp 317-320

Beer J, Siegenthaler U, Oeschger H, Bonani G, Finkel RC (1988) Information on past solar activity and geomagnetism from ${ }^{10} \mathrm{Be}$ in the Camp Century ice core. Nature 331:675-679. doi:10.1038/331675a0

Beer J, Blinov A, Bonani G, Finkel RC, Hofmann HJ, Lehmann B, Oeschger H, Sigg A, Schwander J, Staffelbach T, Stauffer B, Suter M, Wötfli W (1990) Use of ${ }^{10}$ Be in polar ice to trace the 11-year cycle of solar activity. Nature 347:164-166. doi:10.1038/347164a0

Beer J, Tobias S, Weiss N (1998) An active Sun throughout the Maunder minimum. Solar Phys 181:237-249. doi:10.1023/A:1005026001784

Beer J, Vonmoos MV, Muscheler R, McCracken KG, Mende W (2003) Heliospheric modulation over the past 10,000 years as derived from cosmogenic nuclides. In: Kajita T, Asaoka Y, Kawachi A, Matsubara Y, Sasaki M (eds) 28th International cosmic ray conference, vol 7. Universal Academy Press, Tokyo, pp 4147-4150

Beer J, McCracken KG, von Steiger R (2012) Cosmogenic radionuclides: theory and applications in the terrestrial and space environments, physics of earth and space environments, vol 26. Springer, Berlin. doi:10.1007/978-3-642-14651-0

Belmaker R, Lazar B, Tepelyakov N, Stein M, Beer J (2008) ${ }^{10}$ Be in Lake Lisan sediments: A proxy for production or climate? Earth Planet Sci Lett 269:447-456. doi:10.1016/j.eps1.2008.02.032

Belov AV, Gushchina RT, Obridko VN, Shelting BD, Yanke VG (2006) Long-term variations of galactic cosmic rays in the past and future from observations of various solar activity characteristics. J Atmos Terr Phys 68:1161-1166. doi:10.1016/j.jastp.2006.01.001

Berggren AM, Beer J, Possnert G, Aldahan A, Kubik P, Christl M, Johnsen SJ, Abreu JA, Vinther BM (2009) A 600-year annual ${ }^{10}$ Be record from the NGRIP ice core, Greenland. Geophys Res Lett 36:L11801. doi:10.1029/2009GL038004

Bezuglov MV, Malyshevsky VS, Malykhina TV, Torgovkin AV, Fomin GV, Shramenko BI (2012) Photonuclear channel of ${ }^{7}$ Be production in the Earth's atmosphere. Phys Atom Nucl 75:393-397. doi:10. 1134/S1063778812030040

Bhandari PA, Lal D, Rama (1966) Stratospheric circulation studies based on natural and artificail radioactive tracer elements. Tellus 18:391-406. doi:10.3402/tellusa.v18i2-3.9390

Bisschoff D, Potgieter MS (2016) New local interstellar spectra for protons, helium and carbon derived from PAMELA and Voyager 1 observations. Astrophys Space Sci 361:48. doi:10.1007/s10509-015-2633-8. arXiv: 1512.04836

Bonino G, Cini-Castagnoli G, Bhandari N, Taricco C (1995) Behavior of the heliosphere over prolonged solar quiet periods by ${ }^{44} \mathrm{Ti}$ measurements in meteorites. Science 270:1648-1650. doi:10.1126/science. 270.5242 .1648

Bracewell RN (1986) Simulating the sunspot cycle. Nature 323:516-519. doi:10.1038/323516a0

Brandenburg A, Sokoloff D (2002) Local and nonlocal magnetic diffusion and alpha-effect tensors in shear flow turbulence. Geophys Astrophys Fluid Dyn 96:319-344. doi:10.1080/03091920290032974. arXiv:astro-ph/0111568 
Brandenburg A, Spiegel EA (2008) Modeling a Maunder minimum. Astron Nachr 329:351-358. doi:10. 1002/asna.200810973. arXiv:0801.2156

Brandenburg A, Krause F, Meinel R, Moss D, Tuominen I (1989) The stability of nonlinear dynamos and the limited role of kinematic growth rates. Astron Astrophys 213:411-422

Bray RJ, Loughhead RE (1964) Sunspots, international astrophysics series, vol 7. Chapman and Hall, London

Broeker WS, Peng TH (1986) Carbon cycle: 1985 Glacial to interglacial changes in the operation of the global carbon cycle. Radiocarbon 28:309-327. doi:10.1017/S0033822200007414

Brooke JM, Moss D (1994) Non-linear dynamos in torus geometry: transition to chaos. Mon Not R Astron Soc 266:733-739. doi:10.1093/mnras/266.3.733

Burger RA, Potgieter MS, Heber B (2000) Rigidity dependence of cosmic ray proton latitudinal gradients measured by Ulysses spacecraft: Implications for the diffusion tensor. J Geophys Res 105:27,44727,455. doi:10.1029/2000JA000153

Bushby PJ, Tobias SM (2007) On predicting the solar cycle using mean-field models. Astrophys J 661:12891296. doi:10.1086/516628. arXiv:0704.2345

Caballero-Lopez RA, Moraal H (2004) Limitations of the force field equation to describe cosmic ray modulation. J Geophys Res 109:A01101. doi:10.1029/2003JA010098

Calisto M, Usoskin I, Rozanov E, Peter T (2011) Influence of Galactic Cosmic Rays on atmospheric composition and dynamics. Atmos Chem Phys 11:4547-4556. doi:10.5194/acp-11-4547-2011

Cane HV, Lario D (2006) An introduction to CMEs and energetic particles. Space Sci Rev 123:45-56. doi:10.1007/s11214-006-9011-3

Carbonell M, Oliver R, Ballester JL (1993) On the asymmetry of solar activity. Astron Astrophys 274:497504

Carbonell M, Oliver R, Ballester JL (1994) A search for chaotic behaviour in solar activity. Astron Astrophys 290:983-994

Carrasco VMS, Vaquero JM, Gallego MC, Trigo RM (2013) Forty two years counting spots: solar observations by D.E. Hadden during 1890-1931 revisited. New Astron 25:95-102. doi:10.1016/j.newast. 2013.05.002

Casas R, Vaquero JM, Vázquez M (2006) Solar rotation in the 17th century. Solar Phys 234:379-392. doi:10.1007/s11207-006-0036-2

Castagnoli G, Lal D (1980) Solar modulation effects in terrestrial production of carbon-14. Radiocarbon 22:133-158. doi:10.1017/S0033822200009413

Cauquoin A, Raisbeck GM, Jouzel J, Bard E (2014) No evidence for planetary influence on solar activity 330,000 years ago. Astron Astrophys 561:A132. doi:10.1051/0004-6361/201322879

Charbonneau P (2001) Multiperiodicity, chaos, and intermittency in a reduced model of the solar cycle. Solar Phys 199:385-404

Charbonneau P (2010) Dynamo models of the solar cycle. Living Rev Solar Phys 7:Irsp-12010-3. doi:10. 12942/lrsp-2010-3. http://www.livingreviews.org/lrsp-2010-3

Charbonneau P, Dikpati M (2000) Stochastic fluctuations in a Babcock-Leighton model of the solar cycle. Astrophys J 543:1027-1043. doi:10.1086/317142

Charbonneau P, Blais-Laurier G, St-Jean C (2004) Intermittency and phase persistence in a BabcockLeighton model of the solar cycle. Astrophys J Lett 616:L183-L186. doi:10.1086/426897

Choudhuri AR (1992) Stochastic fluctuations of the solar dynamo. Astron Astrophys 253:277-285

Choudhuri AR, Chatterjee P, Jiang J (2007) Predicting solar cycle 24 with a solar dynamo model. Phys Rev Lett 98:131103. doi:10.1103/PhysRevLett.98.131103. arXiv:astro-ph/0701527

Clark DH, Stephenson FR (1978) An interpretation of the pre-telescopic sunspot records from the orient. Q J R Astron Soc 19:387-410

Clette F, Berghmans D, Vanlommel P, van der Linden RAM, Koeckelenbergh A, Wauters L (2007) From the Wolf number to the International Sunspot Index: 25 years of SIDC. Adv Space Res 40:919-928. doi:10.1016/j.asr.2006.12.045

Clette F, Svalgaard L, Vaquero JM, Cliver EW (2014) Revisiting the Sunspot number. A 400-year perspective on the solar cycle. Space Sci Rev 186:35-103. doi:10.1007/s11214-014-0074-2. arXiv:1407.3231

Cliver EW, Ling AG (2016) The discontinuity circa 1885 in the group sunspot number. Solar Phys 291:27632784. doi:10.1007/s11207-015-0841-6

Cliver EW, Boriakoff V, Bounar KH (1998) Geomagnetic activity and the solar wind during the Maunder Minimum. Geophys Res Lett 25:897-900. doi:10.1029/98GL00500 
Cliver EW, Tylka AJ, Dietrich WF, Ling AG (2014) On a solar origin for the cosmogenic nuclide event of 775 AD. Astrophys J 781:32. doi:10.1088/0004-637X/781/1/32

Conway AJ (1998) Time series, neural networks and the future of the Sun. New Astron Rev 42:343-394. doi:10.1016/S1387-6473(98)00041-4

Cooke DJ, Humble JE, Shea MA, Smart DF, Lund N, Rasmussen IL, Byrnak B, Goret P, Petrou N (1991) On cosmic-ray cut-off terminology. Nuovo Cimento C 14:213-234. doi:10.1007/BF02509357

Covas E, Tavakol R, Tworkowski A, Brandenburg A (1998) Axisymmetric mean field dynamos with dynamic and algebraic $\alpha$-quenching. Astron Astrophys 329:350-360 arXiv:astro-ph/9709062

Damon PE, Sonett CP (1991) Solar and terrestrial components of the atmospheric ${ }^{14} \mathrm{C}$ variation spectrum. In: Sonett CP, Giampapa MS, Matthews MS (eds) The sun in time. University of Arizona Press, Tucson, pp 360-388

Damon PE, Lerman JC, Long A (1978) Temporal fluctuations of atmospheric ${ }^{14} \mathrm{C}$ : causal factors and implications. Annu Rev Earth Planet Sci 6:457-494. doi:10.1146/annurev.ea.06.050178.002325

Delaygue G, Bard E (2011) An Antarctic view of Beryllium-10 and solar activity for the past millennium. Clim Dyn 36:2201-2218. doi:10.1007/s00382-010-0795-1

de Carvalho JX, Prado CPC (2000) Self-organized criticality in the Olami-Feder-Christensen model. Phys Rev Lett 84:4006-4009. doi:10.1103/PhysRevLett.84.4006

de Dudok Wit T, Lefèvre L, Clette F (2016) Uncertainties in the sunspot numbers: estimation and implications. Solar Phys 291:2709-2731. doi:10.1007/s11207-016-0970-6. arXiv:1608.05261

de Jong AFM, Mook WG (1982) An anomalous Suess effect above Europe. Nature 298:641-644. doi:10. $1038 / 298641 \mathrm{a} 0$

de Meyer F (1998) Modulation of the solar magnetic cycle. Solar Phys 181:201-219. doi:10.1023/A: 1005079132329

Diercke A, Arlt R, Denker C (2015) Digitization of sunspot drawings by Spörer made in 1861-1894. Astron Nachr 336:53-62. doi:10.1002/asna.201412138. arXiv:1411.7790

Dikpati M, Gilman PA (2006) Simulating and predicting solar cycles using a flux-transport dynamo. Astrophys J 649:498-514. doi:10.1086/506314

Dikpati M, de Toma G, Gilman PA (2008) Polar flux, cross-equatorial flux, and dynamo-generated Tachocline Toroidal flux as predictors of solar cycles. Astrophys J 675:920-930. doi:10.1086/524656

Donadini F, Korte M, Constable C (2010) Millennial variations of the geomagnetic field: from data recovery to field reconstruction. Space Sci Rev 155:219-246. doi:10.1007/s11214-010-9662-y

Donnelly RF, White OR, Livingston WC (1994) The solar Ca II K index and the Mg II core-to-wing ratio. Solar Phys 152:69-76. doi:10.1007/BF01473185

Dorman LI (2004) Cosmic rays in the earth's atmosphere and underground. Kluwer Academic Publishers, Dordrecht

Dreschhoff GAM, Zeller EJ (1990) Evidence of individual solar proton events in Antarctic snow. Solar Phys 127:333-346. doi:10.1007/BF00152172

Dreschhoff GAM, Zeller EJ (1998) Ultra-high resolution nitrate in polar ice as indicator of past solar activity. Solar Phys 177:365-374. doi:10.1023/A:1004932530313

Duderstadt KA, Dibb JE, Schwadron NA, Spence HE, Solomon SC, Yudin VA, Jackman CH, Randall CE (2016) Nitrate ion spikes in ice cores not suitable as proxies for solar proton events. J Geophys Res 121:2994-3016. doi:10.1002/2015JD023805. arXiv:1511.03358

Eddy JA (1976) The Maunder minimum. Science 192:1189-1202. doi:10.1126/science.192.4245.1189

Eddy JA (1977a) Climate and the changing sun. Clim Change 1:173-190. doi:10.1007/BF01884410

Eddy JA (1977b) The case of the missing sunspots. Sci Am 236:80-88. doi:10.1038/ scientificamerican0577-80

Eddy JA (1983) The Maunder minimum: a reappraisal. Solar Phys 89:195-207. doi:10.1007/BF00211962

Eddy JA, Stephenson FR, Yau KKC (1989) On pre-telescopic sunspot records. Q J R Astron Soc 30:65-73

Eichler D, Mordecai D (2012) Comet encounters and carbon 14. Astrophys J Lett 761:L27. doi:10.1088/ 2041-8205/761/2/L27

Elsasser W, Nay EP, Winkler JR (1956) Cosmic-ray intensity and geomagnetism. Nature 178:1226-1227. doi:10.1038/1781226a0

Feynman J, Gabriel SB (1990) Period and phase of the 88-year solar cycle and the Maunder minimum: evidence for a chaotic Sun. Solar Phys 127:393-403. doi:10.1007/BF00152176

Field CV, Schmidt GA, Koch D, Salyk C (2006) Modeling production and climate-related impacts on ${ }^{10} \mathrm{Be}$ concentration in ice cores. J Geophys Res 111:D15107. doi:10.1029/2005JD006410 
Fink D, Klein J, Middleton R, Vogt S, Herzog GF, Reedy RC $(1998){ }^{41} \mathrm{Ca},{ }^{26} \mathrm{Al}$, and ${ }^{10} \mathrm{Be}$ in lunar basalt 74275 and ${ }^{10} \mathrm{Be}$ in the double drive tube 74002/74001. Geochim Cosmochim Acta 62:2389-2402. doi:10.1016/S0016-7037(98)00134-3

Finkel RC, Nishiizumi K (1997) Beryllium 10 concentrations in the Greenland Ice Sheet Project 2 ice core from 3-40 ka. J Geophys Res 102:26,699-26,706. doi:10.1029/97JC01282

Foukal P (1996) The behavior of solar magnetic plages measured from Mt. Wilson observations between 1915-1984. Geophys Res Lett 23:2169-2172. doi:10.1029/96GL01356

Foukal P, Fröhlich C, Spruit H, Wigley TML (2006) Variations in solar luminosity and their effect on the Earth's climate. Nature 443:161-166. doi:10.1038/nature05072

Fraser-Smith AC (1987) Centered and eccentric geomagnetic dipoles and their poles, 1600-1985. Rev Geophys 25:1-16. doi:10.1029/RG025i001p00001

Frick P, Galyagin D, Hoyt DV, Nesme-Ribes E, Schatten KH, Sokoloff D, Zakharov V (1997) Wavelet analysis of solar activity recorded by sunspot groups. Astron Astrophys 328:670-681

Friedli TK (2016) Sunspot observations of Rudolf Wolf from 1849-1893. Solar Phys 291:2505-2517. doi:10.1007/s11207-016-0907-0

Fröhlich C (2006) Solar irradiance variability since 1978. Space Sci Rev 125:53-65

Fröhlich C (2012) Total solar irradiance observations. Surv Geophys 33:453-473. doi:10.1007/ s10712-011-9168-5

Garcia-Munoz M, Mason GM, Simpson JA (1975) The anomalous ${ }^{4} \mathrm{He}$ component in the cosmic-ray spectrum at $\lesssim 50 \mathrm{MeV}$ per nucleon during 1972-1974. Astrophys J 202:265-275. doi:10.1086/153973

Genevey A, Gallet Y, Constable CG, Korte M, Hulot G (2008) ArcheoInt: an upgraded compilation of geomagnetic field intensity data for the past ten millennia. Geochem Geophys Geosyst 9:Q04038. doi:10.1029/2007GC001881

Gerber S, Joos F, Brügger P, Stocker TF, Mann ME, Sitch S, Scholze M (2002) Constraining temperature variations over the last millennium by comparing simulated and observed atmospheric $\mathrm{CO}_{2}$. Clim Dyn 20:281-299

Gibson SE, de Toma G, Emery B, Riley P, Zhao L, Elsworth Y, Leamon RJ, Lei J, McIntosh S, Mewaldt RA, Thompson BJ, Webb D (2011) The whole heliosphere interval in the context of a long and structured solar minimum: an overview from sun to earth. Solar Phys 274:5-27. doi:10.1007/s11207-011-9921-4

Gleeson LJ, Axford WI (1968) Solar modulation of galactic cosmic rays. Astrophys J 154:1011-1026. doi:10.1086/149822

Gleissberg W (1939) A long-periodic fluctuation of the sun-spot numbers. Observatory 62:158-159

Gleissberg W (1948) A preliminary forecast of solar activity. Pop Astron 56:399

Gleissberg W (1952) Die Häufigkeit der Sonnenflecken. Akademie-Verlag, Berlin

Gleissberg W (1971) The probable behaviour of sunspot cycle 21. Solar Phys 21:240-245. doi:10.1007/ BF00155794

Goslar T (2001) Absolute production of radiocarbon and the long-term trend of atmospheric radiocarbon. Radiocarbon 43:743-749. doi:10.1017/S0033822200041400

Goslar T (2003) ${ }^{14} \mathrm{C}$ as an indicator of solar variability. PAGES News 11(2/3):12-14

Gray LJ, Beer J, Geller M, Haigh JD, Lockwood M, Matthes K, Cubasch U, Fleitmann D, Harrison G, Hood L, Luterbacher J, Meehl GA, Shindell D, van Geel B, White W (2010) Solar influences on climate. Rev Geophys 48:RG4001. doi:10.1029/2009RG000282

Grieder PKF (2001) Cosmic rays at earth: researcher's reference manual and data book. Elsevier, Amsterdam

Grismore R, Llewellyn RA, Brown MD, Dowson ST, Cumblidge K (2001) Measurements of the concentrations of ${ }^{26} \mathrm{Al}$ in lunar rocks 15555 and 60025. Earth Planet Sci Lett 187:163-171. doi:10.1016/ S0012-821X(01)00271-0

Güttler D, Beer J, Bleicher N, Boswijk G, Hogg AG, Palmer JG, Wacker L, Wunder J (2013) The 774/775 AD event in the southern hemisphere. In: Laboratory of ion beam physics: annual report 2013, ETH, Zurich, p 33. http://www.ams.ethz.ch/publications/annual_reports/2013

Haigh JD (2007) The sun and the earth's climate. Living Rev Solar Phys 4:1rsp-2007-2. doi:10.12942/ lrsp-2007-2. http://www.livingreviews.org/lrsp-2007-2

Haigh JD, Winning AR, Toumi R, Harder JW (2010) An influence of solar spectral variations on radiative forcing of climate. Nature 467:696-699. doi:10.1038/nature09426

Hale GE, Ellerman F, Nicholson SB, Joy AH (1919) The magnetic polarity of sun-spots. Astrophys J 49:153-178. doi:10.1086/142452

Hambaryan VV, Neuhäuser R (2013) A Galactic short gamma-ray burst as cause for the ${ }^{14} \mathrm{C}$ peak in AD 774/5. Mon Not R Astron Soc. doi:10.1093/mnras/sts378 
Hanslmeier A, Brajša R, Čalogović J, Vršnak B, Ruždjak D, Steinhilber F, MacLeod CL, Ivezić Ž, Skokić I (2013) The chaotic solar cycle. II. Analysis of cosmogenic ${ }^{10} \mathrm{Be}$ data. Astron Astrophys 550:A6. doi:10.1051/0004-6361/201015215

Harvey KL, White OR (1999) What is solar cycle minimum? J Geophys Res 104(A9):19,759-19,764. doi:10.1029/1999JA900211

Hathaway DH (2009) Solar cycle forecasting. Space Sci Rev 144:401-412. doi:10.1007/ s11214-008-9430-4

Hathaway DH (2015) The Solar Cycle. Living Rev Solar Phys 12:1rsp-2015-4, doi:10.1007/1rsp-2015-4 . http://www.livingreviews.org/lrsp-2015-4. arXiv:1502.07020

Hathaway DH, Wilson RM (2004) What the sunspot record tells us about space climate. Solar Phys 224:519. doi:10.1007/s11207-005-3996-8

Hathaway DH, Wilson RM, Reichmann EJ (1999) A synthesis of solar cycle prediction techniques. J Geophys Res 104:22,375-22,388. doi:10.1029/1999JA900313

Heikkilä U, Beer J, Feichter J (2008) Modeling cosmogenic radionuclides ${ }^{10} \mathrm{Be}$ and ${ }^{7} \mathrm{Be}$ during the Maunder minimum using the ECHAM5-HAM general circulation model. Atmos Chem Phys 8:2797-2809. doi:10.5194/acp-8-2797-2008. http://www.atmos-chem-phys.net/8/2797/2008/

Heikkilä U, Beer J, Feichter J (2009) Meridional transport and deposition of atmospheric ${ }^{10}$ Be. Atmos Chem Phys 9:515-527. doi:10.5194/acp-9-515-2009 http://www.atmos-chem-phys.net/9/515/2009/

Herbst K, Kopp A, Heber B, Steinhilber F, Fichtner H, Scherer K, Matthiä D (2010) On the importance of the local interstellar spectrum for the solar modulation parameter. J Geophys Res 115:D00I20. doi:10. 1029/2009JD012557

Herbst K, Heber B, Kopp A, Sternal O, Steinhilber F (2012) The local interstellar spectrum beyond the heliopause: What can be learned from voyager in the inner heliosheath? Astrophys J 761:17. doi:10. 1088/0004-637X/761/1/17

Horiuchi K, Minoura K, Kobayashi K, Nakamura T, Hatori S, Matsuzaki H, Kawai T (1999) Last-glacial to post-glacial ${ }^{10}$ Be fluctuations in a sediment core from the Academician Ridge, Lake Baikal. Geophys Res Lett 26:1047-1050. doi:10.1029/1999GL900163

Horiuchi K, Ohta A, Uchida T, Matsuzaki H, Shibata Y, Motoyama H (2007) Concentration of ${ }^{10}$ Be in an ice core from the Dome Fuji station, Eastern Antarctica: Preliminary results from 1500 to $1810 \mathrm{yr}$ AD. Nucl Instrum Methods B 259:584-587. doi:10.1016/j.nimb.2007.01.306

Horiuchi K, Uchida T, Sakamoto Y, Ohta A, Matsuzaki H, Shibata Y, Motoyama H (2008) Ice core record of ${ }^{10} \mathrm{Be}$ over the past millennium from Dome Fuji, Antarctica: a new proxy record of past solar activity and a powerful tool for stratigraphic dating. Quat Geochronol 3:253-261. doi:10.1016/j.quageo.2008. 01.003

Hoyng P (1993) Helicity fluctuations in mean field theory: An explanation for the variability of the solar cycle? Astron Astrophys 272:321-339

Hoyt DV, Schatten K (1998) Group sunspot numbers: a new solar activity reconstruction. Solar Phys 179:189-219

Hoyt DV, Schatten KH (1996) How well was the Sun observed during the Maunder minimum? Solar Phys 165:181-192. doi:10.1007/BF00149097

Hoyt DV, Schatten KH, Nesme-Ribes E (1994) The one hundredth year of Rudolf Wolf's death: Do we have the correct reconstruction of solar activity? Geophys Res Lett 21:2067-2070. doi:10.1029/94GL01698

Hudson HS (2010) Solar flares add up. Nat Phys 6:637-638. doi:10.1038/nphys1764

Inceoglu F, Simoniello R, Knudsen VF, Karoff C, Olsen J, Turck-Chiéze S, Jacobsen BH (2015) Grand solar minima and maxima deduced from ${ }^{10} \mathrm{Be}$ and ${ }^{14} \mathrm{C}$ : magnetic dynamo configuration and polarity reversal. Astron Astrophys 577:A20. doi:10.1051/0004-6361/201424212

Jennings RL, Weiss NO (1991) Symmetry breaking in stellar dynamos. Mon Not R Astron Soc 252:249-260. doi:10.1093/mnras/252.2.249

Jiang J, Chatterjee P, Choudhuri AR (2007) Solar activity forecast with a dynamo model. Mon Not R Astron Soc 381:1527-1542. doi:10.1111/j.1365-2966.2007.12267.x. arXiv:0707.2258

Joos F (1994) Imbalance in the budget. Nature 370:181-182. doi:10.1038/370181a0

Jull AJT, Cloudt S, Donahue DJ, Sisterson JM, Reedy RC, Masarik J (1998) ${ }^{14}$ C depth profiles in Apollo 15 and 17 cores and lunar rock 68815. Geochim Cosmochim Acta 62:3025-3036. doi:10.1016/ S0016-7037(98)00193-8

Jull AJT, Panyushkina IP, Lange TE, Kukarskih VV, Myglan VS, Clark KJ, Salzer MW, Burr GS, Leavitt SW (2014) Excursions in the ${ }^{14} \mathrm{C}$ record at AD 774-775 in tree rings from Russia and America. Geophys Res Lett 41:3004-3010. doi:10.1002/2014GL059874 
Kane RP (2007) A preliminary estimate of the size of the coming solar cycle 24, based on Ohl's precursor method. Solar Phys 243:205-217. doi:10.1007/s11207-007-0475-4

Käpylä MJ, Käpylä PJ, Olspert N, Brandenburg A, Warnecke J, Karak BB, Pelt J (2016) Multiple dynamo modes as a mechanism for long-term solar activity variations. Astron Astrophys 589:A56. doi:10. 1051/0004-6361/201527002. arXiv:1507.05417

Käpylä PJ, Mantere MJ, Brandenburg A (2012) Cyclic magnetic activity due to turbulent convection in spherical wedge geometry. Astrophys J Lett 755:L22. doi:10.1088/2041-8205/755/1/L22

Karak BB, Nandy D (2012) Turbulent pumping of magnetic flux reduces solar cycle memory and thus impacts predictability of the Sun's activity. Astrophys J Lett 761:L13. doi:10.1088/2041-8205/761/ 1/L13. arXiv:1206.2106

Kigoshi K, Hasegawa H (1966) Secular variation of atmospheric radiocarbon concentration and its dependence on geomagnetism. J Geophys Res 71:1065-1071. doi:10.1029/JZ071i004p01065

Klecker B, Kunow H, Cane HV, Dalla S, Heber B, Kecskemety K, Klein KL, Kóta J, Kucharek H, Lario D, Lee MA, Popecki MA, Posner A, Rodriguez-Pacheco J, Sanderson T, Simnett GM, Roelof EC (2006) Energetic particle observations. Space Sci Rev 123:217-250. doi:10.1007/s11214-006-9018-9

Kleczek J (1952) Solar flare index calculations. Publ Centr Astron Inst Czechoslovakia 22:1

Knudsen MF, Riisager P, Donadini F, Snowball I, Muscheler R, Korhonen K, Pesonen LJ (2008) Variations in the geomagnetic dipole moment during the Holocene and the past 50 kyr. Earth Planet Sci Lett 272:319-329. doi:10.1016/j.eps1.2008.04.048

Koch D, Schmidt GA, Field CV (2006) Sulfur, sea salt, and radionuclide aerosols in GISS ModelE. J Geophys Res 111(D10):D06206. doi:10.1029/2004JD005550

Kocharov GE, Ostryakov VM, Peristykh AN, Vasil'ev VA (1995) Radiocarbon content variations and Maunder minimum of solar activity. Solar Phys 159:381-391. doi:10.1007/BF00686539

Kohl CP, Murrell MT, Russ III GP, Arnold JR (1978) Evidence for the constancy of the solar cosmic ray flux over the past ten million years: ${ }^{53} \mathrm{Mn}$ and ${ }^{26} \mathrm{Al}$ measurements. In: Lunar and planetary science IX, Pergamon Press, New York, Geochim Cosmochim Acta Suppl, vol 10, pp 2299-2310

Korte M, Constable CG (2005) The geomagnetic dipole moment over the last 7000 years-new results from a global model. Earth Planet Sci Lett 236:348-358. doi:10.1016/j.eps1.2004.12.031

Korte M, Constable CG (2006) Centennial to millennial geomagnetic secular variation. Geophys J Int 167:43-52. doi:10.1111/j.1365-246X.2006.03088.x

Korte M, Constable CG (2008) Spatial and temporal resolution of millennial scale geomagnetic field models. Adv Space Res 41:57-69. doi:10.1016/j.asr.2007.03.094

Korte M, Constable C, Donadini F, Holme R (2011) Reconstructing the Holocene geomagnetic field. Earth Planet Sci Lett 312:497-505. doi:10.1016/j.epsl.2011.10.031

Kovaltsov GA, Usoskin IG (2007) Regional cosmic ray induced ionization and geomagnetic field changes. Adv Geosci 13:31-35. doi:10.5194/adgeo-13-31-2007. http://www.adv-geosci.net/13/31/2007/

Kovaltsov GA, Usoskin IG (2010) A new 3D numerical model of cosmogenic nuclide ${ }^{10}$ Be production in the atmosphere. Earth Planet Sci Lett 291:182-188. doi:10.1016/j.eps1.2010.01.011

Kovaltsov GA, Usoskin IG (2014) Occurrence probability of large solar energetic particle events: assessment from data on cosmogenic radionuclides in Lunar rocks. Solar Phys 289:211-220. doi:10.1007/ s11207-013-0333-5. arXiv:1305.5088

Kovaltsov GA, Usoskin IG, Mursula K (2004) An upper limit on sunspot activity during the Maunder minimum. Solar Phys 224:95-101. doi:10.1007/s11207-005-4281-6

Kovaltsov GA, Mishev A, Usoskin IG (2012) A new model of cosmogenic production of radiocarbon ${ }^{14} \mathrm{C}$ in the atmosphere. Earth Planet Sci Lett 337:114-120. doi:10.1016/j.epsl.2012.05.036. arXiv:1206.6974

Kremliovsky MN (1994) Can we understand time scales of solar activity? Solar Phys 151:351-370. doi:10. 1007/BF00679081

Kremliovsky MN (1995) Limits of predictability of solar activity. Solar Phys 159:371-380. doi:10.1007/ BF00686538

Krivova NA, Balmaceda L, Solanki SK (2007) Reconstruction of solar total irradiance since 1700 from the surface magnetic flux. Astron Astrophys 467:335-346. doi:10.1051/0004-6361:20066725

Křivský L (1984) Long-term fluctuations of solar activity during the last thousand years. Solar Phys 93:189194

Křivský L, Pejml K (1988) Solar activity, aurorae and climate in Central Europe in the last 1000 years. Publ Astron Inst Czechoslovak Acad Sci 75:32

Kuklin GV (1976) cyclical and secular variations of solar activity. In: Bumba V, Kleczek J (eds) Basic mechanisms of solar activity, D. Reidel, vol 71. IAU Symposium, Dordrecht, pp 147-148 
Kurths J, Ruzmaikin AA (1990) On forecasting the sunspot numbers. Solar Phys 126:407-410. doi:10. 1007/BF00153060

Lal D (1987) ${ }^{10} \mathrm{Be}$ in polar ice: data reflect changes in cosmic ray flux or polar meteorology. Geophys Res Lett 14:785-788. doi:10.1029/GL014i008p00785

Lal D, Peters B (1967) Cosmic ray produced radioactivity on the Earth. In: Sittle K (ed) Kosmische Strahlung II/cosmic rays II, Handbuch der Physik/Encyclopedia of physics, vol 9/46/2. Springer, Berlin, pp 551612. doi:10.1007/978-3-642-46079-1_7

Lal D, Suess HE (1968) The radioactivity of the atmosphere and hydrosphere. Annu Rev Nucl Sci 18:407434. doi:10.1146/annurev.ns.18.120168.002203

Lal D, Jull AJT, Pollard D, Vacher L (2005) Evidence for large century time-scale changes in solar activity in the past $32 \mathrm{Kyr}$, based on in-situ cosmogenic ${ }^{14} \mathrm{C}$ in ice at Summit, Greenland. Earth Planet Sci Lett 234:335-349. doi:10.1016/j.eps1.2005.02.011

Lawrence JK, Cadavid AC, Ruzmaikin AA (1995) Turbulent and chaotic dynamics underlying solar magnetic variability. Astrophys J 455:366. doi:10.1086/176583

Lee EH, Ahn YS, Yang HJ, Chen KY (2004) The sunspot and auroral activity cycle derived from Korean historical records of the 11th 18th century. Solar Phys 224:373-386. doi:10.1007/s11207-005-5199-8

Letfus V (1999) Daily relative sunspot numbers 1749-1848: reconstruction of missing observations. Solar Phys 184:201-211. doi:10.1023/A:1005086320594

Letfus V (2000) Relative sunspot numbers in the first half of eighteenth century. Solar Phys 194:175-184

Leussu R, Usoskin IG, Arlt R, Mursula K (2013) Inconsistency of the Wolf sunspot number series around 1848. Astron Astrophys 559:A28. doi:10.1051/0004-6361/201322373. arXiv:1310.8443

Li KJ, Yun HS, Gu XM (2001) Latitude migration of sunspot groups. Astron J 122:2115-2117. doi:10. $1086 / 323089$

Licht A, Hulot G, Gallet Y, Thébault E (2013) Ensembles of low degree archeomagnetic field models for the past three millennia. Phys Earth Planet Inter 224:38-67. doi:10.1016/j.pepi.2013.08.007

Lingenfelter RE (1963) Production of carbon 14 by cosmic-ray neutrons. Rev Geophys Space Phys 1:35-55. doi:10.1029/RG001i001p00035

Lingenfelter RE, Hudson HS (1980) Solar particle fluxes and the ancient sun. In: Pepin RO, Eddy JA, Merrill RB (eds) The ancient sun: fossil record in the Earth, Moon and Meteorites. Pergamon Press, New York, pp 69-79

Liu Y, Zhang Z, Peng Z, Ling M, Shen C, Liu W, Sun X, Shen C, Liu K, Sun W (2014) Mysterious abrupt carbon-14 increase in coral contributed by a comet. Sci Rep 4:3728. doi:10.1038/srep03728

Lockwood M (2013) Reconstruction and prediction of variations in the open solar magnetic flux and interplanetary conditions. Liv Rev Solar Phys 10:lrsp-2013-4. doi:10.12942/lrsp-2013-4. http://www. livingreviews.org/lrsp-2013-4

Lockwood M, Owens MJ (2014) Implications of the recent low solar minimum for the solar wind during the Maunder minimum. Astrophys J Lett 781:L7. doi:10.1088/2041-8205/781/1/L7

Lockwood M, Stamper R, Wild MN (1999) A doubling of the Sun's coronal magnetic field during the past 100 years. Nature 399:437-439. doi:10.1038/20867

Lockwood M, Owens MJ, Barnard L, Davis CJ, Steinhilber F (2011) The persistence of solar activity indicators and the descent of the Sun into Maunder minimum conditions. Geophys Res Lett 38:L22105. doi:10.1029/2011GL049811

Lockwood M, Barnard L, Nevanlinna H, Owens MJ, Harrison RG, Rouillard AP, Davis CJ (2013) Reconstruction of geomagnetic activity and near-Earth interplanetary conditions over the past 167 yr - Part 1: A new geomagnetic data composite. Annal Geophys 31:1957-1977. doi:10.5194/ angeo-31-1957-2013

Lockwood M, Owens MJ, Barnard L (2014) Centennial variations in sunspot number, open solar flux, and streamer belt width: 1. Correction of the sunspot number record since 1874. J Geophys Res Space Phys 119:5172-5182. doi:10.1002/2014JA019970

Lockwood M, Owens MJ, Barnard L (2014) Centennial variations in sunspot number, open solar flux, and streamer belt width: 2. Comparison with the geomagnetic data. J Geophys Res 119:5183-5192. doi:10. 1002/2014JA019972

Lockwood M, Owens MJ, Barnard L, Scott CJ, Usoskin IG, Nevanlinna H (2016a) Tests of sunspot number sequences: 2. Using geomagnetic and auroral data. Solar Phys 291:2811-2828. doi:10.1007/ s11207-016-0913-2. arXiv:1605.01948

Lockwood M, Owens MJ, Barnard L, Usoskin IG (2016b) An assessment of sunspot number data composites over 1845-2014. Astrophys J 824:54. doi:10.3847/0004-637X/824/1/54. arXiv:1604.04538 
Lockwood M, Owens MJ, Barnard L, Usoskin IG (2016c) Tests of sunspot number sequences: 3. Effects of regression procedures on the calibration of historic sunspot data. Solar Phys 291:2829-2841. doi:10. 1007/s11207-015-0829-2. arXiv:1510.07809

Love JJ (2011) Secular trends in storm-level geomagnetic activity. Ann Geophys 29:251-262. doi:10.5194/ angeo-29-251-2011

Lukianova R, Alekseev G, Mursula K (2009) Effects of station relocation in the aa index. J Geophys Res 114:A02105. doi:10.1029/2008JA013824

Maehara H, Shibayama T, Notsu S, Notsu Y, Nagao T, Kusaba S, Honda S, Nogami D, Shibata K (2012) Superflares on solar-type stars. Nature 485:478-481. doi:10.1038/nature11063

Martin-Puertas C, Matthes K, Brauer A, Muscheler R, Hansen F, Petrick C, Aldahan A, Possnert G, van Geel B (2012) Regional atmospheric circulation shifts induced by a grand solar minimum. Nature Geosci 5:397-401. doi:10.1038/ngeo1460

Masarik J, Beer J (1999) Simulation of particle fluxes and cosmogenic nuclide production in the Earth's atmosphere. J Geophys Res 104(D10):12,099-12,112. doi:10.1029/1998JD200091

Masarik J, Beer J (2009) An updated simulation of particle fluxes and cosmogenic nuclide production in the Earth's atmosphere. J Geophys Res 114(D13):D11103. doi:10.1029/2008JD010557

Mayaud PN (1972) The $a a$ indices: A 100-year series characterizing the magnetic activity. J Geophys Res 77:6870-6874. doi:10.1029/JA077i034p06870

McCracken K, Beer J (2007) Long-term changes in the cosmic ray intensity at Earth, 1428-2005. J Geophys Res 112(A11):A10,101. doi:10.1029/2006JA012117

McCracken KG (2004) Geomagnetic and atmospheric effects upon the cosmogenic ${ }^{10} \mathrm{Be}$ observed in polar ice. J Geophys Res 109(A18):A04101. doi:10.1029/2003JA010060

McCracken KG (2007) Heliomagnetic field near Earth, 1428-2005. J Geophys Res 112:A09106. doi:10. 1029/2006JA012119

McCracken KG, Dreschhoff GAM, Zeller EJ, Smart DF, Shea MA (2001) Solar cosmic ray events for the period 1561-1994: 1. Identification in polar ice, 1561-1950. J Geophys Res 106:21,585-21,598. doi:10.1029/2000JA000237

McCracken KG, McDonald FB, Beer J, Raisbeck GM, Yiou F (2004) A phenomenological study of the long-term cosmic ray modulation, 850-1958 AD. J Geophys Res 109(A18):A12103. doi:10.1029/ 2004JA010685

McDonald FB, Webber WR, Reames DV (2010) Unusual time histories of galactic and anomalous cosmic rays at 1 AU over the deep solar minimum of cycle 23/24. Geophys Res Lett 37:L18101. doi:10.1029/ 2010GL044218

McHargue LR, Damon PE (1991) The global beryllium 10 cycle. Rev Geophys 29:141-158. doi:10.1029/ 91RG00072

Mega MS, Allegrini P, Grigolini P, Latora V, Palatella L, Rapisarda A, Vinciguerra S (2003) Power-law time distribution of large earthquakes. Phys Rev Lett 90:188501. doi:10.1103/PhysRevLett.90.188501. arXiv:cond-mat/0212529

Mekhaldi F, Muscheler R, Adolphi F, Aldahan A, Beer J, McConnell J, Possnert G, Sigl M, Svensson A, Synal H, Welten K, Woodruff T (2015) Multiradionuclide evidence for the solar origin of the cosmic-ray events of AD 774/5 and 993/4. Nature Commun 6:8611. doi:10.1038/ncomms9611

Melott AL, Thomas BC (2012) Causes of an AD 774-775 ${ }^{14} \mathrm{C}$ increase. Nature 491:E1-E2. doi:10.1038/ nature11695. arXiv: 1212.0490

Mendoza B (1997) Geomagnetic activity and wind velocity during the Maunder minimum. Ann Geophys 15:397-402. doi:10.1007/s00585-997-0397-3. http://www.ann-geophys.net/15/397/1997/

Mewaldt RA (2006) Solar energetic particle composition, energy spectra, and space weather. Space Sci Rev 124:303-316. doi:10.1007/s11214-006-9091-0

Mewaldt RA, Cohen CMS, Mason GM, Haggerty DK, Desai MI (2007) Long-term fluences of solar energetic particles from H to Fe. Space Sci Rev 130:323-328. doi:10.1007/s11214-007-9200-8

Mewaldt RA, Looper MD, Cohen CMS, Haggerty DK, Labrador AW, Leske RA, Mason GM, Mazur JE, von Rosenvinge TT (2012) Energy spectra, composition, and other properties of ground-level events during solar cycle 23. Space Sci Rev 171:97-120. doi:10.1007/s11214-012-9884-2

Michel R, Neumann S (1998) Interpretation of cosmogenic nuclides in meteorites on the basis of accelerator experiments and physical model calculations. Proc Indian Acad Sci (Earth Planet Sci) 107:441-457

Michel R, Leya I, Borges L (1996) Production of cosmogenic nuclides in meteoroids: accelerator experiments and model calculations to decipher the cosmic ray record in extraterrestrial matter. Nucl Instrum Methods B 113:434-444. doi:10.1016/0168-583X(95)01345-8 
Mikaloff Fletcher SE, Gruber N, Jacobson AR, Doney SC, Dutkiewicz S, Gerber M, Follows M, Joos F, Lindsay K, Menemenlis D, Mouchet A, Müller SA, Sarmiento JL (2006) Inverse estimates of anthropogenic $\mathrm{CO}_{2}$ uptake, transport, and storage by the ocean. Global Biogeochem Cycles 20:GB2002. doi:10.1029/2005GB002530

Mininni PD, Gómez DO, Mindlin GB (2000) Stochastic relaxation oscillator model for the solar cycle. Phys Rev Lett 85:5476-5479. doi:10.1103/PhysRevLett.85.5476

Mininni PD, Gómez DO, Mindlin GB (2001) Simple model of a stochastically excited solar dynamo. Solar Phys 201:203-223. doi:10.1023/A:1017515709106

Mininni PD, Gómez DO, Mindlin GB (2002) Biorthogonal decomposition techniques unveil the nature of the irregularities observed in the solar cycle. Phys Rev Lett 89(6):061101. doi:10.1103/PhysRevLett. 89.061101

Mironova IA, Aplin KL, Arnold F, Bazilevskaya GA, Harrison RG, Krivolutsky AA, Nicoll KA, Rozanov EV, Turunen E, Usoskin IG (2015) Energetic particle influence on the earth's atmosphere. Space Sci Rev 194:1-96. doi:10.1007/s11214-015-0185-4

Mishev AL, Usoskin IG, Kovaltsov GA (2013) Neutron monitor yield function: new improved computations. J Geophys Res 118:2783-2788. doi:10.1002/jgra.50325

Miyahara H, Masuda K, Muraki Y, Furuzawa H, Menjo H, Nakamura T (2004) Cyclicity of solar activity during the Maunder minimum deduced from radiocarbon content. Solar Phys 224:317-322. doi:10. 1007/s11207-005-6501-5

Miyahara H, Masuda K, Muraki Y, Kitagawa H, Nakamura T (2006a) Variation of solar cyclicity during the Spoerer minimum. J Geophys Res 111(A10):A03103. doi:10.1029/2005JA011016

Miyahara H, Sokoloff D, Usoskin IG (2006b) The solar cycle at the Maunder minimum epoch. In: Ip WH, Duldig M (eds) Advances in geosciences, solar terrestrial (ST), vol 2. World Scientific, Singapore, pp $1-20$

Miyahara H, Kitazawa K, Nagaya K, Yokoyama Y, Matsuzaki H, Masuda K, Nakamura T, Muraki Y (2010) Is the Sun heading for another Maunder minimum? Precursors of the grand solar minima. J Cosmol 8:1970-1982

Miyake F, Nagaya K, Masuda K, Nakamura T (2012) A signature of cosmic-ray increase in ad 774-775 from tree rings in Japan. Nature 486:240-242. doi:10.1038/nature11123

Miyake F, Masuda K, Nakamura T (2013) Another rapid event in the carbon-14 content of tree rings. Nature Commun 4:1748. doi:10.1038/ncomms2783

Miyake F, Suzuki A, Masuda K, Horiuchi K, Motoyama H, Matsuzaki H, Motizuki Y, Takahashi K, Nakai $\mathrm{Y}$ (2015) Cosmic ray event of A.D. 774-775 shown in quasi-annual ${ }^{10} \mathrm{Be}$ data from the Antarctic Dome Fuji ice core. Geophys Res Lett 42:84-89. doi:10.1002/2014GL062218

Miyake F, Masuda K, Nakamura T, Kimura K, Hakozaki M, Jull T, Lange T, Cruz R, Panyushkina I, Baisan C, Salzer M (2016) Search for annual ${ }^{14} \mathrm{C}$ excursions in the past. Radiocarbon. doi:10.1017/RDC. 2016.54

Moraal H, Stoker PH (2010) Long-term neutron monitor observations and the 2009 cosmic ray maximum. J Geophys Res 115:A12109. doi:10.1029/2010JA015413

Morfill GE, Scheingraber H, Voges W, Sonett CP (1991) Sunspot number variations: stochastic or chaotic. In: Sonett CP, Giampapa MS, Matthews MS (eds) The sun in time. University of Arizona Press, Tucson, pp 30-58

Moss D, Brandenburg A, Tavakol R, Tuominen I (1992) Stochastic effects in mean-field dynamos. Astron Astrophys 265:843-849

Moss D, Sokoloff D, Usoskin IG, Tutubalin V (2008) Solar grand minima and random fluctuations in dynamo parameters. Solar Phys 250:221-234. doi:10.1007/s11207-008-9202-z. arXiv:0806.3331

Mossman JE (1989) A comprehensive search for sunspots without the aid of a telescope, 1981-1982. Quart J R Astron Soc 30:59-64

Mundt MD, Maguire WB II, Chase RRP (1991) Chaos in the sunspot cycle: analysis and prediction. J Geophys Res 96:1705-1716. doi:10.1029/90JA02150

Mursula K, Martini D (2006) Centennial increase in geomagnetic activity: latitudinal differences and global estimates. J Geophys Res 111:A08209. doi:10.1029/2005JA011549

Mursula K, Usoskin IG, Kovaltsov GA (2001) Persistent 22-year cycle in sunspot activity: evidence for a relic solar magnetic field. Solar Phys 198:51-56. doi:10.1023/A:1005218414790

Mursula K, Usoskin IG, Kovaltsov GA (2003) Reconstructing the long-term cosmic ray intensity: linear relations do not work. Ann Geophys 21:863-867. doi:10.5194/angeo-21-863-2003. http://www. ann-geophys.net/21/863/2003/ 
Muscheler R, Joos F, Müller SA, Snowball I (2005) How unusual is today’s solar activity? Nature 436:E3E4. doi:10.1038/nature04045

Muscheler R, Joos F, Beer J, Müller SA, Vonmoos M, Snowball I (2007) Solar activity during the last 1000 yr inferred from radionuclide records. Quat Sci Rev 26:82-97. doi:10.1016/j.quascirev.2006.07.012

Nagaya K, Kitazawa K, Miyake F, Masuda K, Muraki Y, Nakamura T, Miyahara H, Matsuzaki H (2012) Variation of the Schwabe cycle length during the grand solar minimum in the 4th century BC deduced from radiocarbon content in tree rings. Solar Phys 280:223-236. doi:10.1007/s11207-012-0045-2

Nagovitsyn YA (1997) A nonlinear mathematical model for the solar cyclicity and prospects for reconstructing the solar activity in the past. Astron Lett 23:742-748

Nevalainen J, Usoskin IG, Mishev A (2013) Eccentric dipole approximation of the geomagnetic field: application to cosmic ray computations. Adv Space Res 52:22-29. doi:10.1016/j.asr.2013.02.020

Nevanlinna H (1995) Auroral observations in Finland-visual sightings during the 18th and 19th centuries. J Geomagn Geoelectr 47:953-960. doi:10.5636/jgg.47.953

Nevanlinna H (2004a) Historical space climate data from Finland: compilation and analysis. Solar Phys 224:395-405. doi:10.1007/s11207-005-3749-8

Nevanlinna H (2004) Results of the Helsinki magnetic observatory 1844-1912. Ann Geophys 22:16911704. doi:10.5194/angeo-22-1691-2004. http://www.ann-geophys.net/22/1691/2004/

Newton HW (1928) The Sun's cycle of activity. Quart J R Meteorol Soc 54(227):161-174

Ney EP (1959) Cosmic radiation and the weather. Nature 183:451-452. doi:10.1038/183451a0

Nilsson A, Holme R, Korte M, Suttie N, Hill M (2014) Reconstructing holocene geomagnetic field variation: new methods, models and implications. Geophys J Int 198:229-248. doi:10.1093/gji/ggu120

Nishiizumi K, Arnold JR, Kohl CP, Caffee MW, Masarik J, Reedy RC (2009) Solar cosmic ray records in lunar rock 64455. Geochim Cosmochim Acta 73:2163-2176. doi:10.1016/j.gca.2008.12.021

Nishizumi K, Caffee MW, Arnold JR (1997) ${ }^{10}$ Be from the active Sun. In: Lunar and planetary science XXVIII, Lunar and Planetary Institute, Houston

O'Brien K (1979) Secular variations in the production of cosmogenic isotopes in the Earth's atmosphere. J Geophys Res 84:423-431. doi:10.1029/JA084iA02p00423

O'Brien K, Burke GDP (1973) Calculated cosmic ray neutron monitor response to solar modulation of galactic cosmic rays. J Geophys Res 78:3013-3019. doi:10.1029/JA078i016p03013

Oeschger H, Siegenthaler U, Schotterer U, Gugelmann A (1974) A box diffusion model to study the carbon dioxide exchange in nature. Tellus 27:168-192

Ogurtsov MG (2004) New evidence for long-term persistence in the Sun's activity. Solar Phys 220:93-105. doi:10.1023/B:sola.0000023439.59453.e5

Ogurtsov MG, Nagovitsyn YA, Kocharov GE, Jungner H (2002) Long-period cycles of the Sun's activity recorded in direct solar data and proxies. Solar Phys 211:371-394. doi:10.1023/A:1022411209257

Oguti T, Egeland A (1995) Auroral occurrence in Norwegian archives. J Geomagn Geoelectr 47:353-359. doi:10.5636/jgg.47.353

Oliver R, Ballester JL (1996) Rescaled range analysis of the asymmetry of solar activity. Solar Phys 169:215224. doi:10.1007/BF00153842

Oliver R, Ballester JL (1998) Is there memory in solar activity? Phys Rev E 58:5650-5654. doi:10.1103/ PhysRevE.58.5650

Ossendrijver MAJH (2000) The dynamo effect of magnetic flux tubes. Astron Astrophys 359:1205-1210

Ossendrijver MAJH (2003) The solar dynamo. Astron Astrophys Rev 11:287-367. doi:10.1007/ s00159-003-0019-3

Ossendrijver MAJH, Hoyng P, Schmitt D (1996) Stochastic excitation and memory of the solar dynamo. Astron Astrophys 313:938-948

Ostryakov VM, Usoskin IG (1990a) Correlation dimensions of structured signals. Sov Tech Phys Lett 16:658-659

Ostryakov VM, Usoskin IG (1990b) On the dimension of solar attractor. Solar Phys 127:405-412. doi:10. 1007/BF00152177

Owens M, Cliver E, McCracken K, Beer J, Barnard L, Lockwood M, Rouillard A, Passos D, Riley P, Usoskin I, Wang YM (2016) Near-earth heliospheric magnetic field intensity since 1750. Part 2: Cosmogenic radionuclide reconstructions. J Geophys Res (in press), doi:10.1002/2016JA022550

Owens MJ, Usoskin IG, Lockwood M (2012) Heliospheric modulation of galactic cosmic rays during grand solar minima: past and future variations. Geophys Res Lett 39:L19102. doi:10.1029/2012GL053151

Özgüç A, Ataç T, Rybák J (2003) Temporal variability of the flare index (1966-2001). Solar Phys 214:375396. doi:10.1023/A:1024225802080 
Panchev S, Tsekov M (2007) Empirical evidences of persistence and dynamical chaos in solar terrestrial phenomena. J Atmos Sol Terr Phys 69:2391-2404. doi:10.1016/j.jastp.2007.07.011

Parker EN (1955) Hydromagnetic dynamo models. Astrophys J 122:293-314. doi:10.1086/146087

Parker EN (1965) The passage of energetic charged particles through interplanetary space. Planet Space Sci 13:9-49. doi:10.1016/0032-0633(65)90131-5

Pavlov AK, Blinov AV, Konstantinov AN, Ostryakov VM, Vasilyev GI, Vdovina MA, Volkov PA (2013) AD 775 pulse of cosmogenic radionuclides production as imprint of a Galactic gamma-ray burst. Mon Notes R Astron Soc 435:2878-2884. doi:10.1093/mnras/stt1468

Pavón-Carrasco FJ, Osete ML, Torta JM, De Santis A (2014) A geomagnetic field model for the Holocene based on archaeomagnetic and lava flow data. Earth Planet Sci Lett 388:98-109. doi:10.1016/j.epsl. 2013.11.046

Pedro JB, Heikkilä UE, Klekociuk A, Smith AM, van Ommen TD, Curran MAJ (2011) Beryllium-10 transport to Antarctica: results from seasonally resolved observations and modeling. J Geophys Res 116:D23120. doi:10.1029/2011JD016530

Peristykh AN, Damon PE (1998) Modulation of atmospheric ${ }^{14} \mathrm{C}$ concentration by the solar wind and irradiance components of the Hale and Schwabe solar cycles. Solar Phys 177:343-343. doi:10.1023/ A:1004982321191

Peristykh AN, Damon PE (2003) Persistence of the Gleissberg 88-year solar cycle over the last $\sim 12,000$ years: evidence from cosmogenic isotopes. J Geophys Res 108:1003. doi:10.1029/2002JA009390

Pesnell WD (2012) Solar cycle predictions (Invited Review). Solar Phys 281:507-532. doi:10.1007/ s11207-012-9997-5

Pesnell WD (2016) Predictions of solar cycle 24: How are we doing? Space Weather 14(1):10-21. doi:10. 1002/2015SW001304

Petrovay K (2010) Solar cycle prediction. Living Rev Solar Phys 7:1rsp-2010-6. doi:10.12942/lrsp-2010-6 arXiv: 1012.5513

Pipin VV, Sokoloff DD, Usoskin IG (2012) Variations of the solar cycle profile in a solar dynamo with fluctuating dynamo governing parameters. Astron Astrophys 542:A26. doi:10.1051/0004-6361/201118733. arXiv:1112.6218

Poluianov S, Usoskin IG (2014) Critical analysis of a hypothesis of the planetary tidal influence on solar activity. Solar Phys 289:2333-2342. doi:10.1007/s11207-014-0475-0. arXiv:1401.3547

Poluianov S, Kovaltsov G, Mishev A, Usoskin IG (2016) Production of cosmogenic isotopes 7Be, 10Be, $14 \mathrm{C}, 22 \mathrm{Na}$ and $36 \mathrm{Cl}$ in the atmosphere: Altitudinal profiles of yield functions. J Geophys Res 121:(in press). doi:10.1002/2016JD025034, arXiv:1606.05899

Polygiannakis J, Preka-Papadema P, Moussas X (2003) On signal-noise decomposition of time-series using the continuous wavelet transform: application to sunspot index. Mon Not R Astron Soc 343:725-734. doi:10.1046/j.1365-8711.2003.06705.x. arXiv:physics/0301030

Potgieter M (2013) Solar modulation of cosmic rays. Living Rev Solar Phys 10:1rsp.2013-3. doi:10.12942/ lrsp-2013-3 arXiv:1306.4421

Price CP, Prichard D, Hogenson EA (1992) Do the sunspot numbers form a 'chaotic' set? J Geophys Res 97:19,113-19,120. doi:10.1029/92JA01459

Pulkkinen T (2007) Space weather: terrestrial perspective. Living Rev Solar Phys 4:1rsp-2007-1. doi:10. 12942/lrsp-2007-1. http://www.livingreviews.org/lrsp-2007-1

Raisbeck GM, Yiou F, Fruneau M, Loiseaux JM, Lieuvin M, Ravel JC (1981) Cosmogenic ${ }^{10} \mathrm{Be} /{ }^{7} \mathrm{Be}$ as a probe of atmospheric transport processes. Geophys Res Lett 8:1015-1018. doi:10.1029/ GL008i009p01015

Raisbeck GM, Yiou F, Jouzel J, Petit JR (1990) ${ }^{10} \mathrm{Be}$ and $\delta{ }^{2} \mathrm{H}$ in polar ice cores as a probe of the solar variability's influence on climate. Philos Trans R Soc London Ser A 330:463-469. doi:10.1098/rsta. 1990.0027

Rao MN, Garrison DH, Bogard DD, Reedy RC (1994) Determination of the flux and energy distribution of energetic solar protons in the past 2 Myr using lunar rock 68815. Geochim Cosmochim Acta 58:4231-4245. doi:10.1016/0016-7037(94)90275-5

Reames DV, Ng CK (2010) Streaming-limited intensities of solar energetic particles on the intensity plateau. Astrophys J 723:1286-1293. doi:10.1088/0004-637X/723/2/1286

Reedy RC (1996) Constraints on solar particle events from comparisons of recent events and million-year averages. In: Balasubramaniam KS, Keil SL, Smartt RN (eds) Solar drivers of the interplanetary and terrestrial disturbances, astronomical society of the Pacific, San Francisco, ASP Conference Series, vol 95, pp 429-436 
Reedy RC (2002) Recent solar energetic particles: updates and trends. In: 33rd Lunar and planetary science conference, Lunar and Planetary Institute, Houston. http://www.lpi.usra.edu/meetings/lpsc2002/pdf/ 1938.pdf

Reedy RC (2012) Update on solar-proton fluxes during the last five solar activity cycles. In: 43rd Lunar and Planetary Science Conference, Lunar and Planetary Institute, Houston, vol 43. http://www.lpi.usra. edu/meetings/lpsc2012/pdf/1285.pdf

Reeves GD, Cayton TE, Gary SP, Belian RD (1992) The great solar energetic particle events of 1989 observed from geosynchronous orbit. J Geophys Res 97:6219-6226. doi:10.1029/91JA03102

Reimer PJ, Baillie MGL, Bard E, Bayliss A, Beck JW, Blackwell PG, Ramsey CB, Buck CE, Burr GS, Edwards RL, Friedrich M, Grootes PM, Guilderson TP, Hajdas I, Heaton TJ, Hogg AG, Hughen KA, Kaiser KF, Kromer B, McCormac FG, Manning SW, Reimer RW, Richards DA, Southon JR, Talamo S, Turney CSM, van der Plicht J, Weyhenmeyer CE (2009) IntCal09 and Marine09 radiocarbon age calibration curves, 0-50,000 years cal BP. Radiocarbon 51(4):1111-1150. doi:10.1017/ S0033822200034202. https://journals.uair.arizona.edu/index.php/radiocarbon/article/view/3569

Reimer PJ, Bard E, Bayliss A, Beck JW, Blackwell PG, Ramsey CB, Buck CE, Cheng H, Edwards RL, Friedrich M, Grootes PM, Guilderson TP, Haflidason H, Hajdas I, Hatte C, Heaton TJ, Hoffmann DL, Hogg AG, Hughen KA, Kaiser KF, Kromer B, Manning SW, Niu M, Reimer RW, Richards DA, Scott EM, Southon JR, Staff RA, Turney CSM, van der Plicht J (2013) IntCal13 and Marine13 radiocarbon age calibration curves 0-50,000 years cal BP. Radiocarbon 55(4):1869-1887. doi:10.2458/azu_js_rc. 55.16947

Ribes JC, Nesme-Ribes E (1993) The solar sunspot cycle in the Maunder minimum AD1645 to AD1715. Astron Astrophys 276:549-563

Rigozo NR, Echer E, Vieira LEA, Nordemann DJR (2001) Reconstruction of Wolf sunspot numbers on the basis of spectral characteristics and estimates of associated radio flux and solar wind parameters for the last Millennium. Solar Phys 203:179-191. doi:10.1023/A:1012745612022

Roth R, Joos F (2013) A reconstruction of radiocarbon production and total solar irradiance from the Holocene ${ }^{14} \mathrm{C}$ and $\mathrm{CO}_{2}$ records: implications of data and model uncertainties. Clim Past 9:18791909. doi:10.5194/cp-9-1879-2013

Rozanov E, Calisto M, Egorova T, Peter T, Schmutz W (2012) Influence of the precipitating energetic particles on atmospheric chemistry and climate. Surv Geophys 33:483-501. doi:10.1007/ s10712-012-9192-0

Rozelot JP (1994) On the stability of the 11-year solar cycle period (and a few others). Solar Phys 149:149154. doi:10.1007/BF00645186

Rozelot JP (1995) On the chaotic behaviour of the solar activity. Astron Astrophys 297:L45-L48

Ruth U, Barnola JM, Beer J, Bigler M, Blunier T, Castellano E, Fischer H, Fundel F, Huybrechts P, Kaufmann P, Kipfstuhl S, Lambrecht A, Morganti A, Oerter H, Parrenin F, Rybak O, Severi M, Udisti R, Wilhelms F, Wolff EW (2007) 'EDML1': a chronology for the EPICA deep ice core from Dronning Maud Land, Antarctica, over the last 150000 years. 3:475-484. doi:10.5194/cp-3-475-2007. http://www.clim-past. net/3/475/2007/

Ruzmaikin A, Feynman J, Robinson P (1994) Long-term persistence of solar activity. Solar Phys 149:395403. doi:10.1007/BF00690625

Ruzmaikin AA (1981) The solar cycle as a strange attractor. Comments Astrophys 9:85-93

Rybanský M, Rušin V, Minarovjech M, Klocok L, Cliver EW (2005) Reexamination of the coronal index of solar activity. J Geophys Res 110(A9):8106. doi:10.1029/2005JA011146

Sabine CL, Feely RA, Gruber N, Key RM, Lee K, Bullister JL, Wanninkhof R, Wong CS, Wallace DWR, Tilbrook B, Millero FJ, Peng TH, Kozyr A, Ono T, Rios AF (2004) The Oceanic sink for anthropogenic $\mathrm{CO}_{2}$. Science 305:367-371. doi:10.1126/science.1097403

Salakhutdinova II (1999) Identifying the quasi-regular and stochastic components of solar cyclicity and their properties. Solar Phys 188:377-396. doi:10.1023/A:1005265229175

Sarychev AP, Roshchina EM (2009) Comparison of three solar activity indices based on sunspot observations. Solar Syst Res 43:151-157. doi:10.1134/S0038094609020087

Scherer K, Fichtner H (2004) Constraints on the heliospheric magnetic field variation during the Maunder Minimum from cosmic ray modulation modelling. Astron Astrophys 413:L11-L14. doi:10.1051/ 0004-6361:20034636

Scherer K, Fahr HJ, Fichtner H, Heber B (2004) Long-term modulation of cosmic rays in the heliosphere and its influence at earth. Solar Phys 224:305-316. doi:10.1007/s11207-005-5687-X

Schlamminger L (1990) Aurora borealis during the Maunder minimum. Mon Not R Astron Soc 247:67-69 
Schmalz S, Stix M (1991) An $\alpha \Omega$ dynamo with order and chaos. Astron Astrophys 245:654-661

Schmidt GA, Jungclaus JH, Ammann CM, Bard E, Braconnot P, Crowley TJ, Delaygue G, Joos F, Krivova NA, Muscheler R, Otto-Bliesner BL, Pongratz J, Shindell DT, Solanki SK, Steinhilber F, Vieira LEA (2012) Climate forcing reconstructions for use in PMIP simulations of the Last Millennium (v1.1). Geosci Model Dev 5:185-191. doi:10.5194/gmd-5-185-2012. http://www.geosci-model-dev.net/5/ $185 / 2012 /$

Schmitt D, Schüssler M, Ferriz-Mas A (1996) Intermittent solar activity by an on-off dynamo. Astron Astrophys 311:L1-L4

Schove DJ (1955) The sunspot cycle, 649 B.C. to A.D. 2000. J Geophys Res 60:127-146. doi:10.1029/ JZ060i002p00127

Schove DJ (1979) Sunspot turning-points and aurorae since A.D. 1510. Solar Phys 63:423-432. doi:10. 1007/BF00174546

Schove DJ (1983) Sunspot, auroral, radiocarbon and climatic fluctuations since 7000 BC. Ann Geophys $1: 391-396$

Schrijver CJ, Beer J, Baltensperger U, Cliver EW, Güdel M, Hudson HS, McCracken KG, Osten RA, Peter T, Soderblom DR, Usoskin IG, Wolff EW (2012) Estimating the frequency of extremely energetic solar events, based on solar, stellar, lunar, and terrestrial records. J Geophys Res 117:A08103. doi:10. 1029/2012JA017706

Schröder W (1992) On the existence of the 11-year cycle in solar and auroral activity before and after the so-called Maunder minimum. J Geomagn Geoelectr 44:119-128. doi:10.5636/jgg.44.119

Schüpbach S, Federer U, Bigler M, Fischer H, Stocker TF (2011) A refined TALDICE-1a age scale from 55 to $112 \mathrm{ka}$ before present for the Talos Dome ice core based on high-resolution methane measurements. Clim Past 7:1001-1009. doi:10.5194/cp-7-1001-2011. http://www.clim-past.net/7/1001/2011/

Schüssler M, Caligari P, Ferriz-Mas A, Moreno-Insertis F (1994) Instability and eruption of magnetic flux tubes in the solar convection zone. Astron Astrophys 281:L69-L72

Schüssler M, Schmitt D, Ferriz-Mas A (1997) Long-term variation of solar activity by a dynamo based on magnetic flux tubes. In: Schmieder B, del Toro Iniesta JC, Vázquez M (eds) 1st Advances in solar physics euroconference: advances in the physics of sunspots, astronomical society of the Pacific, San Francisco, ASP Conference Series, vol 118, pp 39-44

Scuderi LA (1990) Oriental sunspot observations and volcanism. Quart J R Astron Soc 31:109-120

Sello S (2000) Wavelet entropy as a measure of solar cycle complexity. Astron Astrophys 363:311-315 arXiv:astro-ph/0005334

Semeniuk K, Fomichev VI, McConnell JC, Fu C, Melo SML, Usoskin IG (2011) Middle atmosphere response to the solar cycle in irradiance and ionizing particle precipitation. Atmos Chem Phys 11:50455077. doi:10.5194/acp-11-5045-2011. http://www.atmos-chem-phys.net/11/5045/2011/

Senthamizh Pavai V, Arlt R, Dasi-Espuig M, Krivova NA, Solanki SK (2015) Sunspot areas and tilt angles for solar cycles 7-10. Astron Astrophys 584:A73. doi:10.1051/0004-6361/201527080. arXiv:1508.07849

Serre T, Nesme-Ribes E (2000) Nonlinear analysis of solar cycles. Astron Astrophys 360:319-330

Shea MA, Smart DF (1990) A summary of major solar proton events. Solar Phys 127:297-320. doi:10. 1007/BF00152170

Shea MA, Smart DF (2002) Solar proton event patterns: the rising portion of five solar cycles. Adv Space Res 29:325-330. doi:10.1016/S0273-1177(01)00592-0

Shea MA, Smart DF, McCracken KG, Dreschhoff GAM, Spence HE (2006) Solar proton events for 450 years: the Carrington event in perspective. Adv Space Res 38:232-238. doi:10.1016/j.asr.2005.02.100

Shibata K, Isobe H, Hillier A, Choudhuri AR, Maehara H, Ishii TT, Shibayama T, Notsu S, Notsu Y, Nagao T, Honda S, Nogami D (2013) Can superflares occur on our sun? Publ Astron Soc Japan. doi:10.1093/ pasj/65.3.49. arXiv:1212.1361

Shindell D, Rind D, Balachandran N, Lean JL, Lonergan P (1999) Solar cycle variability, ozone, and climate. Science 284:305-308. doi:10.1126/science.284.5412.305

Siegenthaler U, Heimann M, Oeschger H (1980) ${ }^{14} \mathrm{C}$ variations caused by changes in the global carbon cycle. Radiocarbon 22:177-191. doi:10.1017/S0033822200009449

Sigl M, Winstrup M, McConnell JR, Welten KC, Plunkett G, Ludlow F, Büntgen U, Caffee M, Chellman N, Dahl-Jensen D, Fischer H, Kipfstuhl S, Kostick C, Maselli OJ, Mekhaldi F, Mulvaney R, Muscheler R, Pasteris DR, Pilcher JR, Salzer M, Schüpbach S, Steffensen JP, Vinther BM, Woodruff TE (2015) Timing and climate forcing of volcanic eruptions for the past 2,500 years. Nature 523:543-549. doi:10. 1038 /nature 14565 
Silverman SM (1992) Secular variation of the aurora for the past 500 years. Rev Geophys 30:333-351. doi:10.1029/92RG01571

Silverman SM (2006) Comparison of the aurora of September 1/2, 1859 with other great auroras. Adv Space Res 38:136-144. doi:10.1016/j.asr.2005.03.157

Siscoe GL (1980) Evidence in the auroral record for secular solar variability. Rev Geophys Space Phys 18:647-658. doi:10.1029/RG018i003p00647

Siscoe GL, Verosub KL (1983) High medieval auroral incidence over China and Japan: implications for the medieval site of the geomagnetic pole. Geophys Res Lett 10:345-348. doi:10.1029/GL010i004p00345

Smart DF, Shea MA (2002) A review of solar proton events during the 22nd solar cycle. Adv Space Res 30:1033-1044. doi:10.1016/S0273-1177(02)00497-0

Smart DF, Shea MA, Melott AL, Laird CM (2014) Low time resolution analysis of polar ice cores cannot detect impulsive nitrate events. J Geophys Res 119:9430-9440. doi:10.1002/2014JA020378. arXiv: 1501.01204

Snow M, McClintock WE, Woods TN, White OR, Harder JW, Rottman G (2005) The Mg II Index from SORCE. Solar Phys 230:325-344. doi:10.1007/s11207-005-6879-0

Snowball I, Muscheler R (2007) Palaeomagnetic intensity data: an Achilles heel of solar activity reconstructions. Holocene 17:851-859. doi:10.1177/0959683607080531

Sokoloff D (2004) The Maunder minimum and the solar dynamo. Solar Phys 224:145-152. doi:10.1007/ s11207-005-4176-6

Sokoloff D, Nesme-Ribes E (1994) The Maunder minimum: a mixed-parity dynamo mode? Astron Astrophys 288:293-298

Solanki SK, Krivova NA (2004) Solar irradiance variations: from current measurements to long-term estimates. Solar Phys 224:197-208. doi:10.1007/s11207-005-6499-8

Solanki SK, Schüssler M, Fligge M (2000) Evolution of the Sun's large-scale magnetic field since the Maunder minimum. Nature 408:445-447. doi:10.1038/35044027

Solanki SK, Scüssler M, Fligge M (2002) Secular variation of the Sun's magnetic flux. Astron Astrophys 383:706-712. doi:10.1051/0004-6361:20011790

Solanki SK, Usoskin IG, Kromer B, Schüssler M, Beer J (2004) Unusual activity of the Sun during recent decades compared to the previous 11,000 years. Nature 431:1084-1087. doi:10.1038/nature02995

Solanki SK, Usoskin IG, Kromer B, Schüssler M, Beer J (2005) Climate: How unusual is today's solar activity? Reply. Nature 436:E4-E5. doi:10.1038/nature04046

Sonett CP (1983) The great solar anomaly ca. 1780-1800: an error in compiling the record? J Geophys Res 88:3225-3228. doi:10.1029/JA088iA04p03225

Sonett CP, Finney SA (1990) The spectrum of radiocarbon. Philos Trans R Soc London Ser A 330:413-425. doi:10.1098/rsta.1990.0022

Soon WWH, Yaskell SH (2003) The Maunder minimum and the variable sun-earth connection. World Scientific, River Edge

Steig EJ, Polissar PJ, Stuiver M, Grootes PM, Finkel RC (1996) Large amplitude solar modulation cycles of ${ }^{10} \mathrm{Be}$ in Antarctica: implications for atmospheric mixing processes and interpretation of the ice core record. Geophys Res Lett 23:523-526. doi:10.1029/96GL00255

Steinhilber F, Beer J, Fröhlich C (2009) Total solar irradiance during the Holocene. Geophys Res Lett 36:L19704. doi:10.1029/2009GL040142

Steinhilber F, Abreu JA, Beer J, McCracken KG (2010) Interplanetary magnetic field during the past 9300 years inferred from cosmogenic radionuclides. J Geophys Res 115:A01104. doi:10.1029/ 2009JA014193

Steinhilber F, Abreu JA, Beer J, Brunner I, Christl M, Fischer H, Heikkilä U, Kubik PW, Mann M, McCracken KG, Miller H, Miyahara H, Oerter H, Wilhelms F (2012) 9,400 years of cosmic radiation and solar activity from ice cores and tree rings. Proc Natl Acad Sci USA 109(16):5967-5971. doi:10.1073/pnas. 1118965109

Stephenson FR, Willis DM, Hallinan TJ (2004) Aurorae: the earliest datable observation of the aurora borealis. Astron Geophys 45:6.15-6.17. doi:10.1046/j.1468-4004.2003.45615.x

Stuiver M (1961) Variations in radiocarbon concentration and sunspot activity. J Geophys Res 66:273-276. doi:10.1029/JZ066i001p00273

Stuiver M, Braziunas TF (1989) Atmospheric ${ }^{14}$ C and century-scale solar oscillations. Nature 338:405-408. doi: $10.1038 / 338405 \mathrm{a} 0$

Stuiver M, Braziunas TF (1993) Sun, ocean, climate and atmospheric ${ }^{14} \mathrm{CO}_{2}$ : an evaluation of casual and spectral relationships. Holocene 3(4):289-305. doi:10.1177/095968369300300401 
Stuiver M, Pollach H (1977) Discussion: reporting of ${ }^{14} \mathrm{C}$ data. Radiocarbon 19:355-363. doi:10.1017/ S0033822200003672

Stuiver M, Quay PD (1980) Changes in atmospheric carbon-14 attributed to a variable sun. Science 207:1119. doi:10.1126/science.207.4426.11

Stuiver M, Braziunas TF, Becker B, Kromer B (1991) Climatic, solar, oceanic, and geomagnetic influences on late-glacial and holocene atmospheric ${ }^{14} \mathrm{C} /{ }^{12} \mathrm{C}$ change. Quat Res 35:1-24. doi:10.1016/ 0033-5894(91)90091-I

Suess HE (1955) Radiocarbon concentration in modern wood. Science 122:415-417. doi:10.1126/science. 122.3166.415-a

Suess HE (1980) The radiocarbon record in tree rings of the last 8000 years. Radiocarbon 22:200-209. doi:10.1017/S0033822200009462

Svalgaard L (2012) How well do we know the sunspot number? Proc IAU 286:27-33. doi:10.1017/ S1743921312004590

Svalgaard L, Cliver EW (2005) The IDV index: its derivation and use in inferring long-term variations of the interplanetary magnetic field strength. J Geophys Res 110(A9):A12103. doi:10.1029/2005JA011203

Svalgaard L, Schatten KH (2016) Reconstruction of the sunspot group number: the backbone method. Solar Phys 291:2653-2684. doi:10.1007/s11207-015-0815-8. arXiv:1506.00755

Svalgaard L, Cliver EW, Le Sager P (2004) IHV: a new long-term geomagnetic index. Adv Space Res 34:436-439. doi:10.1016/j.asr.2003.01.029

Svensmark H (1998) Influence of cosmic rays on Earth's climate. Phys Rev Lett 81:5027-5030. doi:10. 1103/PhysRevLett.81.5027

Sweeney C, Gloor E, Jacobson AR, Key RM, McKinley G, Sarmiento JL, Wanninkhof R (2007) Constraining global air-sea gas exchange for $\mathrm{CO}_{2}$ with recent bomb ${ }^{14} \mathrm{C}$ measurements. Global Biogeochem Cycles 21:GB2015. doi:10.1029/2006GB002784

Tans PP, de Jong AFM, Mook WG (1979) Natural atmospheric ${ }^{14} \mathrm{C}$ variation and the Suess effect. Nature 280:826-828. doi:10.1038/280826a0

Tapping KF (1987) Recent solar radio astronomy at centimeter wavelengths: the temporal variability of the 10.7-cm flux. J Geophys Res 92:829-838. doi:10.1029/JD092iD01p00829

Tapping KF, Charrois DP (1994) Limits to the accuracy of the $10.7 \mathrm{~cm}$ flux. Solar Phys 150:305-315. doi:10.1007/BF00712892

Taricco C, Bhandari N, Cane D, Colombetti P, Verma N (2006) Galactic cosmic ray flux decline and periodicities in the interplanetary space during the last 3 centuries revealed by ${ }^{44} \mathrm{Ti}$ in meteorites. $\mathrm{J}$ Geophys Res 111:A08102. doi:10.1029/2005JA011459

Temmer M, Veronig A, Hanslmeier A (2002) Hemispheric sunspot numbers $R_{n}$ and $R_{s}$ : catalogue and N-S asymmetry analysis. Astron Astrophys 390:707-715. doi:10.1051/0004-6361:20020758. arXiv:astro-ph/0208436

Thomas BC, Melott AL, Arkenberg KR, Snyder BR (2013) Terrestrial effects of possible astrophysical sources of an AD 774-775 increase in ${ }^{14} \mathrm{C}$ production. Geophys Res Lett 40:1237-1240. doi:10. $1002 /$ grl.50222

Tinsley BA, Zhou L (2006) Initial results of a global circuit model with variable stratospheric and tropospheric aerosols. J Geophys Res 111(D10):D16205. doi:10.1029/2005JD006988

Tobias S, Hughes D, Weiss N (2006) Unpredictable Sun leaves researchers in the dark. Nature 442:26. doi: $10.1038 / 442026 \mathrm{c}$

Tobias SM, Weiss NO, Kirk V (1995) Chaotically modulated stellar dynamos. Mon Not R Astron Soc 273:1150-1166. doi:10.1093/mnras/273.4.1150

Toptygin IN (1985) Cosmic rays in interplanetary magnetic fields. Geophysics and astrophysics monographs. Kluwer Academic Publishers, Dordrecht

Traversi R, Usoskin IG, Solanki SK, Becagli S, Frezzotti M, Severi M, Stenni B, Udisti R (2012) Nitrate in polar ice: a new tracer of solar variability. Solar Phys 280:237-254. doi:10.1007/s11207-012-0060-3

Traversi R, Becagli S, Poluianov S, Severi M, Solanki SK, Usoskin IG, Udisti R (2016) The Laschamp geomagnetic excursion featured in nitrate record from EPICA-Dome C ice core. Sci Rep 6:20235. doi:10.1038/srep20235

Tylka A, Dietrich W (2009) A new and comprehensive analysis of proton spectra in ground-level enhanced (GLE) solar particle events. In: 31th International cosmic ray conference. Universal Academy Press, Lodź

Tylka AJ, Dietrich WF, Boberg PR (1997) Probability distribution of high-energy solar-heavy-ion fluxes from IMP-8: 1973-1996. IEEE Trans Nucl Sci 44:2140-2149. doi:10.1109/23.659029 
Usoskin I, Mursula K, Kovaltsov G (2001a) Was one sunspot cycle lost in late XVIII century? Astron Astrophys 370:L31-L34. doi:10.1051/0004-6361:20010319

Usoskin I, Mursula K, Solanki S, Schüssler M, Kovaltsov G (2002a) A physical reconstruction of cosmic ray intensity since 1610. J Geophys Res 107(A11):1374. doi:10.1029/2002JA009343

Usoskin IG (2011) Cosmic rays and climate forcing. Mem Soc Astron Ital 82:937-942

Usoskin IG, Kovaltsov GA (2004) Long-term solar activity: direct and indirect study. Solar Phys 224:37-47. doi:10.1007/s11207-005-3997-7

Usoskin IG, Kovaltsov GA (2006) Cosmic ray induced ionization in the atmosphere: full modeling and practical applications. J Geophys Res 111:D21206. doi:10.1029/2006JD007150

Usoskin IG, Kovaltsov GA (2008a) Cosmic rays and climate of the Earth: possible connection. Comptes Rendus Geosci 340:441-450. doi:10.1016/j.crte.2007.11.001

Usoskin IG, Kovaltsov GA (2008b) Production of cosmogenic ${ }^{7}$ Be isotope in the atmosphere: full 3D modelling. J Geophys Res 113:D12107. doi:10.1029/2007JD009725

Usoskin IG, Kovaltsov GA (2012) Occurrence of extreme solar particle events: assessment from historical proxy data. Astrophys J 757:92. doi:10.1088/0004-637X/757/1/92. arXiv:1207.5932

Usoskin IG, Kromer B (2005) Reconstruction of the ${ }^{14} \mathrm{C}$ production rate from measured relative abundance. Radiocarbon 47:31-37. doi:10.1017/S0033822200052176

Usoskin IG, Mursula K (2003) Long-term solar cycle evolution: review of recent developments. Solar Phys 218:319-343. doi:10.1023/B:SOLA.0000013049.27106.07

Usoskin IG, Kananen H, Mursula K, Tanskanen P, Kovaltsov GA (1998) Correlative study of solar activity and cosmic ray intensity. J Geophys Res 103(A5):9567-9574. doi:10.1029/97JA03782

Usoskin IG, Mursula K, Kovaltsov GA (2000) Cyclic behaviour of sunspot activity during the Maunder minimum. Astron Astrophys 354:L33-L36

Usoskin IG, Mursula K, Kovaltsov GA (2001b) Heliospheric modulation of cosmic rays and solar activity during the Maunder minimum. J Geophys Res 106:16,039-16,046. doi:10.1029/2000JA000105

Usoskin IG, Mursula K, Kovaltsov GA (2002b) Lost sunspot cycle in the beginning of Dalton minimum: new evidence and consequences. Geophys Res Lett 29:36-1. doi:10.1029/2002GL015640

Usoskin IG, Mursula K, Kovaltsov GA (2003a) Reconstruction of monthly and yearly group sunspot numbers from sparse daily observations. Solar Phys 218:295-305. doi:10.1023/B:SOLA.0000013029. 99907.97

Usoskin IG, Mursula K, Kovaltsov GA (2003b) The lost sunspot cycle: reanalysis of sunspot statistics. Astron Astrophys 403:743-748. doi:10.1051/0004-6361:20030398

Usoskin IG, Solanki SK, Schüssler M, Mursula K, Alanko K (2003c) Millennium-scale sunspot number reconstruction: evidence for an unusually active sun since the 1940s. Phys Rev Lett 91:211101. doi:10. 1103/PhysRevLett.91.211101. arXiv:astro-ph/0310823

Usoskin IG, Mursula K, Solanki SK, Schüssler M, Alanko K (2004) Reconstruction of solar activity for the last millennium using ${ }^{10} \mathrm{Be}$ data. Astron Astrophys 413:745-751. doi:10.1051/0004-6361:20031533. arXiv:astro-ph/0309556

Usoskin IG, Alanko-Huotari K, Kovaltsov GA, Mursula K (2005) Heliospheric modulation of cosmic rays: monthly reconstruction for 1951-2004. J Geophys Res 110:A12108. doi:10.1029/2005JA011250

Usoskin IG, Solanki SK, Korte M (2006a) Solar activity reconstructed over the last 7000 years: the influence of geomagnetic field changes. Geophys Res Lett 33:8103. doi:10.1029/2006GL025921

Usoskin IG, Solanki SK, Kovaltsov GA, Beer J, Kromer B (2006b) Solar proton events in cosmogenic isotope data. Geophys Res Lett 33(L08):107. doi:10.1029/2006GL026059

Usoskin IG, Solanki SK, Taricco C, Bhandari N, Kovaltsov GA (2006c) Long-term solar activity reconstructions: direct test by cosmogenic ${ }^{44} \mathrm{Ti}$ in meteorites. Astron Astrophys 457:L25-L28. doi:10.1051/ 0004-6361:20065803

Usoskin IG, Solanki SK, Kovaltsov GA (2007) Grand minima and maxima of solar activity: new observational constraints. Astron Astrophys 471:301-309. doi:10.1051/0004-6361:20077704. arXiv:0706.0385

Usoskin IG, Korte M, Kovaltsov GA (2008) Role of centennial geomagnetic changes in local atmospheric ionization. Geophys Res Lett 35:L05811. doi:10.1029/2007GL033040

Usoskin IG, Field CV, Schmidt GA, Leppänen AP, Aldahan A, Kovaltsov GA, Possnert G, Ungar RK (2009a) Short-term production and synoptic influences on atmospheric ${ }^{7}$ Be concentrations. J Geophys Res 114(D13):D06108. doi:10.1029/2008JD011333

Usoskin IG, Horiuchi K, Solanki SK, Kovaltsov GA, Bard E (2009b) On the common solar signal in different cosmogenic isotope data sets. J Geophys Res 114(A13):A03112. doi:10.1029/2008JA013888 
Usoskin IG, Mursula K, Arlt R, Kovaltsov GA (2009c) A solar cycle lost in 1793-1800: early sunspot observations resolve the old mystery. Astrophys J Lett 700:L154-L157. doi:10.1088/0004-637X/ 700/2/L154. arXiv:0907.0063

Usoskin IG, Sokoloff D, Moss D (2009d) Grand minima of solar activity and the mean-field dynamo. Solar Phys 254:345-355. doi:10.1007/s11207-008-9293-6

Usoskin IG, Mironova IA, Korte M, Kovaltsov GA (2010) Regional millennial trend in the cosmic ray induced ionization of the troposphere. J Atmos Sol Terr Phys 72:19-25. doi:10.1016/j.jastp.2009.10. 003

Usoskin IG, Bazilevskaya GA, Kovaltsov GA (2011) Solar modulation parameter for cosmic rays since 1936 reconstructed from ground-based neutron monitors and ionization chambers. J Geophys Res 116:A02104. doi:10.1029/2010JA016105

Usoskin IG, Kromer B, Ludlow F, Beer J, Friedrich M, Kovaltsov GA, Solanki SK, Wacker L (2013) The AD775 cosmic event revisited: the Sun is to blame. Astron Astrophys 552:L3. doi:10.1051/0004-6361/ 201321080. arXiv:1302.6897

Usoskin IG, Hulot G, Gallet Y, Roth R, Licht A, Joos F, Kovaltsov GA, Thébault E, Khokhlov A (2014) Evidence for distinct modes of solar activity. Astron Astrophys 562:L10. doi:10.1051/0004-6361/ 201423391. arXiv:1402.4720

Usoskin IG, Arlt R, Asvestari E, Hawkins E, Käpylä M, Kovaltsov GA, Krivova N, Lockwood M, Mursula K, O'Reilly J, Owens M, Scott CJ, Sokoloff DD, Solanki SK, Soon W, Vaquero JM (2015) The Maunder minimum (1645-1715) was indeed a grand minimum: a reassessment of multiple datasets. Astron Astrophys 581:A95. doi:10.1051/0004-6361/201526652. arXiv:1507.05191

Usoskin IG, Gallet Y, Lopes F, Kovaltsov GA, Hulot G (2016a) Solar activity during the Holocene: the Hallstatt cycle and its consequence for grand minima and maxim. Astron Astrophys 587:A150. doi:10. 1051/0004-6361/201527295

Usoskin IG, Kovaltsov GA, Lockwood M, Mursula K, Owens M, Solanki SK (2016b) A new calibrated sunspot group series since 1749: statistics of active day fractions. Solar Phys 291:2685-2708. doi:10. 1007/s11207-015-0838-1. arXiv:1512.06421

Vaquero JM (2007) Historical sunspot observations: a review. Adv Space Res 40:929-941. doi:10.1016/j. asr.2007.01.087. arXiv:astro-ph/0702068

Vaquero JM, Vázquez M (2009) The sun recorded through history: scientific data extracted from historical documents, astrophysics and space science library, vol 361. Springer, Berlin

Vaquero JM, Gallego MC, García JA (2002) A 250-year cycle in naked-eye observations of sunspots. Geophys Res Lett 29:58-1-58-4. doi:10.1029/2002GL014782

Vaquero JM, Gallego MC, Sánchez-Bajo F (2004) Reconstruction of a monthly homogeneous sunspot area series since 1832. Solar Phys 221:179-189. doi:10.1023/B:SOLA.0000033360.67976.bd

Vaquero JM, Trigo RM, Gallego MC (2005) A 'lost' sunspot observation in 1785. Astron Nachr 326:112114. doi:10.1002/asna.200410343

Vaquero JM, Trigo RM, Gallego MC, Moreno-Corral MA (2007) Two early sunspots observers: Teodoro de Almeida and José Antonio Alzate. Solar Phys 240:165-175. doi:10.1007/s11207-006-0264-5

Vaquero JM, Gallego MC, Usoskin IG, Kovaltsov GA (2011) Revisited sunspot data: a new scenario for the onset of the Maunder minimum. Astrophys J Lett 731:L24. doi:10.1088/2041-8205/731/2/L24. arXiv: 1103.1520

Vaquero JM, Trigo RM, Gallego MC (2012) A simple method to check the reliability of annual sunspot number in the historical period 1610-1847. Solar Phys 277:389-395. doi:10.1007/s11207-011-9901-8. arXiv: 1111.2633

Vaquero JM, Gutiérrez-López S, Szelecka A (2014) A note on the relationship between sunspot numbers and active days. Adv Space Res 53:1180-1183. doi:10.1016/j.asr.2014.01.029

Vaquero JM, Gutiérrez-López S, Szelecka A (2014) A note on the relationship between sunspot numbers and active days. Adv Space Res 53:1180-1183. doi:10.1016/j.asr.2014.01.029. arXiv:1401.7037

Vaquero JM, Kovaltsov GA, Usoskin IG, Carrasco VMS, Gallego MC (2015) Level and length of cyclic solar activity during the Maunder minimum as deduced from the active day statistics. Astron Astrophys 577:A71. doi:10.1051/0004-6361/201525962

Vaquero JM, Svalgaard L, Carrasco VMS, Clette F, Lefèvre L, Gallego MC, Arlt R, Aparicio AJP, Richard JG, Howe R (2016) A revised collection of sunspot group numbers. Solar Phys 291:3061-3074. doi:10. 1007/s11207-016-0982-2. arXiv:1609.04882 
Vasiliev SS, Dergachev VA (2002) The 2400-year cycle in atmospheric radiocarbon concentration: bispectrum of ${ }^{14} \mathrm{C}$ data over the last 8000 years. Ann Geophys 20:115-120. doi:10.5194/angeo-20-115-2002. http://www.ann-geophys.net/20/115/2002/

Vasiliev SS, Dergachev VA, Raspopov OM, Jungner H (2012) Long-term variations in the flux of cosmogenic isotope ${ }^{10} \mathrm{Be}$ over the last 10000 years: Variations in the geomagnetic field and climate. Geomagn Aeron 52:121-128. doi:10.1134/S001679321201015X

Vázquez M, Vaquero JM (2010) Aurorae observed at the Canary Islands. Solar Phys 267:431-444. doi:10. 1007/s11207-010-9650-0

Vieira LEA, Solanki SK, Krivova NA, Usoskin IG (2011) Evolution of the solar irradiance during the Holocene. Astron Astrophys 531:A6. doi:10.1051/0004-6361/201015843. arXiv:1103.4958

Viereck RA, Puga LC (1999) The NOAA Mg II core-to-wing solar index: construction of a 20-year time series of chromospheric variability from multiple satellites. J Geophys Res 104(9995-10):006. doi:10. 1029/1998JA900163

Vitinsky YI (1965) Solar activity forecasting. Israel Program for Scientific Translations, Jerusalem

Vitinsky YI, Kopecky M, Kuklin GV (1986) Statistics of sunspot activity. Nauka, Moscow

Vogt S, Herzog GF, Reedy RC (1990) Cosmogenic nuclides in extraterrestrial materials. Rev Geophys 28:253-275. doi:10.1029/RG028i003p00253

Vonmoos MV, Beer J, Muscheler R (2006) Large variations in Holocene solar activity: constraints from ${ }^{10} \mathrm{Be}$ in the Greenland Ice Core Project ice core. J Geophys Res 111(A10):A10105. doi:10.1029/ 2005JA011500

Voss H, Kurths J, Schwarz U (1996) Reconstruction of grand minima of solar activity from $\Delta^{14} \mathrm{C}$ data: linear and nonlinear signal analysis. J Geophys Res 101:15,637-15,644. doi:10.1029/96JA00542

Waldmeier M (1961) The sunspot-activity in the years 1610-1960. Schulthess u. Co./Swiss Federal Observatory, Zürich

Wang YM, Sheeley NR Jr (2012) The solar wind and interplanetary field during very low amplitude sunspot cycles. Astrophys J 764:90. doi:10.1088/0004-637X/764/1/90

Wang YM, Lean J, Sheeley NR Jr (2005) Modeling the Sun's magnetic field and irradiance since 1713. Astrophys J 625:522-538. doi:10.1086/429689

Webber WR, Higbie PR (2003) Production of cosmogenic Be nuclei in the Earth's atmosphere by cosmic rays: its dependence on solar modulation and the interstellar cosmic ray spectrum. J Geophys Res 108:1355. doi:10.1029/2003JA009863

Webber WR, Higbie PR (2009) Galactic propagation of cosmic ray nuclei in a model with an increasing diffusion coefficient at low rigidities: a comparison of the new interstellar spectra with Voyager data in the outer heliosphere. J Geophys Res 114:A02103. doi:10.1029/2008JA013689

Webber WR, Higbie PR, McCracken KG (2007) Production of the cosmogenic isotopes ${ }^{3} \mathrm{H},{ }^{7} \mathrm{Be},{ }^{10} \mathrm{Be}$, and ${ }^{36} \mathrm{Cl}$ in the Earth's atmosphere by solar and galactic cosmic rays. J Geophys Res 112:A10106. doi:10.1029/2007JA012499

Webber WR, Cummings AC, McDonald FB, Stone EC, Heikkila B, Lal N (2008) Galactic cosmic ray H and He nuclei energy spectra measured by Voyagers 1 and 2 near the heliospheric termination shock in positive and negative solar magnetic polarity cycles. J Geophys Res 113(A12):A10108. doi:10.1029/ 2008JA013395

Weiss NO, Tobias SM (2000) Physical causes of solar activity. Space Sci Rev 94:99-112. doi:10.1023/A: 1026790416627

Weiss NO, Cattaneo F, Jones CA (1984) Periodic and aperiodic dynamo waves. Geophys Astrophys Fluid Dyn 30:305-341. doi:10.1080/03091928408219262

Wheatland MS (2003) The coronal mass ejection waiting-time distribution. Solar Phys 214:361-373. doi:10. 1023/A:1024222511574. arXiv:astro-ph/0303019

Willis DM, Stephenson FR (2001) Solar and auroral evidence for an intense recurrent geomagnetic storm during December in AD 1128. Ann Geophys 19:289-302. doi:10.5194/angeo-19-289-2001. http:// www.ann-geophys.net/19/289/2001/

Willis DM, Easterbrook MG, Stephenson FR (1980) Seasonal variation of oriental sunspot sightings. Nature 287:617-619. doi:10.1038/287617a0

Willis DM, Davda VN, Stephenson FR (1996) Comparison between oriental and occidental sunspot observations. Q J R Astron Soc 37:189-229

Willis DM, Wild MN, Warburton JS (2016) Re-examination of the daily number of sunspot groups for the royal observatory, Greenwich (1874-1885). Solar Phys 291:2519-2552. doi:10.1007/ s11207-016-0856-7 
Wilson PR (1994) Solar and stellar activity cycles, Cambridge astrophysics series, vol 24. Cambridge University Press, Cambridge

Wilson RM (1988a) Bimodality and the Hale cycle. Solar Phys 117:269-278. doi:10.1007/BF00147248

Wilson RM (1988b) On the long-term secular increase in sunspot number. Solar Phys 115:397-408. doi:10. $1007 / \mathrm{BF} 00148736$

Wilson RM (1998) A comparison of Wolf's reconstructed record of annual sunspot number with Schwabe's observed record of 'Clusters of Spots' for the interval of 1826-1868. Solar Phys 182:217-230. doi:10. 1023/A:1005046820210

Wittmann AD, Xu ZT (1987) A catalogue of sunspot observations from 165 BC to AD 1684. Astron Astrophys Suppl 70:83-94

Wolff EW, Bigler M, Curran MAJ, Dibb JE, Frey MM, Legrand M, McConnell JR (2012) The Carrington event not observed in most ice core nitrate records. Geophys Res Lett 39:L08503. doi:10.1029/ 2012GL051603

Wolff EW, Bigler M, Curran MAJ, Dibb JE, Frey MM, Legrand M, McConnell JR (2016) Comment on "Low time resolution analysis of polar ice cores cannot detect impulsive nitrate events " by D.F. Smart et al. J Geophys Res 121:1920-1924. doi:10.1002/2015JA021570

Yau KKC, Stephenson FR (1988) A revised catalog of far eastern observations of sunspots (165 BC to AD 1918). Q J R Astron Soc 29:175-197

Yeo KL, Krivova NA, Solanki SK (2014) Solar cycle variation in solar irradiance. Space Sci Rev 186:137167. doi:10.1007/s11214-014-0061-7. arXiv:1407.4249

Yiou F, Raisbeck GM, Baumgartner S, Beer J, Hammer C, Johnsen S, Jouzel J, Kubik PW, Lestringuez J, Stiévenard M, Suter M, Yiou P (1997) Beryllium 10 in the Greenland ice core project ice core at summit, Greenland. J Geophys Res 102:26,783-26,794. doi:10.1029/97JC01265

Yule GU (1927) On a method of investigating periodicities in disturbed series, with special reference to Wolfer's sunspot numbers. Philos Trans R Soc London Ser A 226:267-298. doi:10.1098/rsta.1927. 0007

Zeller EJ, Dreschhoff GAM (1995) Anomalous nitrate concentrations in polar ice cores-do they result from solar particle injections into the polar atmosphere? Geophys Res Lett 22:2521-2524. doi:10.1029/ 95GL02560

Zharkova VV, Shepherd SJ, Popova E, Zharkov SI (2015) Heartbeat of the sun from principal component analysis and prediction of solar activity on a millenium timescale. Sci Rep 5:15689. doi:10.1038/ srep 15689

Zhentao X (1990) Solar observations in ancient china and solar variability. Philos Trans R Soc London Ser A 330:513-516. doi:10.1098/rsta.1990.0032

Zięba S, Nieckarz Z (2014) Sunspot time series: passive and active intervals. Solar Phys 289:2705-2726. doi:10.1007/s11207-014-0498-6

Zolotova NV, Ponyavin DI (2007) Was the unusual solar cycle at the end of the XVIII century a result of phase asynchronization? Astron Astrophys 470:L17-L20. doi:10.1051/0004-6361:20077681

Zolotova NV, Ponyavin DI (2015) The Maunder Minimum is Not as Grand as it Seemed to be. Astrophys J 800:42. doi:10.1088/0004-637X/800/1/42 